\title{
New insights into the strong formulation finite element method for solving elastostatic and elastodynamic problems
}

\begin{abstract}
This present paper has a complete and homogeneous presentation of plane stress and plane strain problems using the Strong Formulation Finite Element Method (SFEM). In particular, a greater emphasis is given to the numerical implementation of the governing and boundary conditions of the partial differential system of equations. The paper's focus is on numerical stability and accuracy related to elastostatic and elastodynamic problems. In the engineering literature, results are mainly reported for isotropic and homogeneous structures. In this paper, a composite structure is investigated. The SFEM solution is compared to the ones obtained using commercial finite element codes. Generally, the SFEM observes fast accuracy and all the results are in very good agreement with the ones presented in literature.
\end{abstract}

Keywords: Elastostatic Problem; Elastodynamic Problem; Composite Structure; Strong Formulation Finite Element Method; Differential Quadrature Method.

DOI 10.2478/cls-2014-0005

Received September 24, 2014 ; accepted October 30, 2014

\section{Introduction}

The elastostatic and elastodynamic problems [1-3] for engineering applications constitute a widely known approach for the study of structural components, laboratory tests, composite materials, composite structures, and so on. Some of the most studied problems of the present class are two-dimensional (2D) models, which find application in several engineering fields, such as solid mechanics, dynamics of structures, fracture mechanics, wave propagation, seismic stability and rock mechanics. It should be

\footnotetext{
*Corresponding Author: Nicholas Fantuzzi: DICAM - Department, School of Engineering and Architecture, University of Bologna, Italy; E-mail: nicholas.fantuzzi@unibo.it; http://software. dicam.unibo.it/diqumaspab-project
}

cited that the mechanics of static and dynamic composite systems has been studied by several researches. Thus, several books have been hitherto published [4-10].

Problems involving the theory of elasticity can be solved analytically only when simple geometries occur. Thus, the most common way of treating these problems is to make numerical models. Generally the elastic behavior of homogeneous and composite solids is studied using the co-called Boundary Value Problems (BVPs). These problems are governed by partial differential equations where the displacement parameters (the unknowns of the model) are the physical displacements in the three-dimensional (3D) space.

Mathematically speaking, all the quantities involved in the formulation must be smooth all over the definition domain. However, discontinuities can occur in practical applications. The most common discontinuations are related to the material and/or the geometry. The former is connected to composite materials such as reinforced fibers immersed into a matrix and the latter is directly connected to cracks and slits. When these cases come out, some mathematical tricks have to be introduced.

The present approach has its roots in the Differential Quadrature (DQ) method [11, 12], which have the peculiarity of being very accurate using a small number of degrees of freedom when compared to classic numerical approaches, such as the Finite Element Method (FEM). It should be mentioned that DQ method belongs to the big family of the Methods of Weighted Residuals (MWRs) [13], since the functional approximation using weighting coefficients must minimize the error between the given function and its approximation. Furthermore, DQ method can be seen as a general form of the so-called collocation methods or spectral collocation $[14,15]$. The original pseudospectral collocation (DQ method) presented by Bellman has been improved by the approach proposed by Quan [16] and by Quan and Chang $[17,18]$. All the advancements in the DQ method has been reviewed by Bert and Malik [19]. The DQ method in its original version had some numerical instabilities, when the number of grid points is large, since 
the weighting coefficient matrix becomes ill-conditioned due to the chosen basis functions (power basis). Following the ideas by Quan and Chang $[17,18]$ a generalized version of the DQ method, known as Generalized Differential Quadrature (GDQ) method, had been developed by Shu [20] and by Shu and Richards [21, 22]. Nowadays, the GDQ method is the most widely used in literature due to its stability and reliability when applied to different engineering problems. The DQ method had demonstrated to be a useful tool for solving structural components [23-32]. Subsequently, the GDQ method showed the same properties, in fact several numerical applications related to structural mechanics and solid mechanics can be found in [33-84]. The GDQ method turned out to be really useful for studying structural components and the mechanics of composite structures, due to the easy implementation of the governing system of equations for one-dimensional (1D) and twodimensional (2D) systems. As it is well-known the GDQ method solves the strong formulation of the differential problem. This implies that the boundary conditions must be enforced a posteriori. On the contrary using a weak (variational) formulation they are a priori satisfied. The GDQ solving system contains algebraic equations, which come from the governing equations (domain equations) and from the boundary equations (boundary conditions).

Despite what someone might think, strong formulation approaches can be used when irregularities are present. In literature two different approaches were presented. The first one is related to the so-called mesh-less methods in which scattered points are chosen in the physical domain [85-109]. The second one is based on the domain decomposition, such as the one used in the classic FEM. Contrarily to the FEM, domain decomposition methods solve the strong formulation inside each element instead of the weak one. Furthermore, the continuity conditions between two elements must be enforced in order to satisfy the connectivity among them. Hence, the accuracy of this approach depends on the predefined mesh used for the discretization other than the approximation inside each element. Historically speaking, researchers divided a regular domain using regular elements, this approach was termed multi-domain differential quadrature [110-115]. Inserting the mapping technique in the previous works the differential quadrature element method (DQEM) or SFEM can be defined [116-125]. The author presented a preliminary development of the present work in his $\mathrm{PhD}$ Thesis [126]. Some other papers followed about the vibration of arbitrary shaped laminated composite plates [127], some comparisons between SFEM and the cell method [128-130]. Moreover, the stress and strain recovery has been published on the static analysis of arbi- trary shaped plates [131, 132]. A review article about the vibration problem of composite membranes has been presented also [133]. A comparison between the SFEM based on GDQ and RBF methods was presented in [134]. The free vibration behavior of arbitrary shaped functionally graded plates was presented in [135]. A first review about the stability and accuracy for the static and free vibration analysis of SFEM was illustrated in [136]. Nevertheless, a complete survey about SFEM and related methods was recently published in [137].

At a first instance mesh-less methods are better since they do not depend on the mesh used. Mesh-less methods generally use local basis functions, which depend on the distance between the points. In fact they are also called radial basis functions. These local functions depend on a parameter (shape parameter) which influences the accuracy of the solution. On the contrary SFEM, based on DQ method, uses global higher-order basis functions in each element, which have a high accuracy, fast convergence and do not depend on any shape parameter.

The present manuscript presents the SFEM for 2D plane stress and strain problems. After a brief introduction on the 2D elasticity, the algebraic equations in extended and in matrix forms are presented. In particular, a wide focus is given to the application of the mapping technique to a single element. Furthermore, some new details about the boundary conditions and inter-element connectivity conditions are given. For the sake of clarity, it is given to the reader a graphical representation of the boundary conditions for a general SFEM mesh. This helps the reader to better understand all the different boundary conditions that should be implemented. Subsequently, a section is dedicated to the assemblage of the solving system in discrete form for both the static and free vibration case. Some numerical tests are performed using some reference solutions in literature and new numerical applications are proposed for further studies on the subject.

\section{Preliminary remarks}

A wide number of applications have been presented throughout several years about structures which are in deformation or tension state. As it is well-known these states are better known as plane strain and plane stress. For instance, if a thin plate is loaded by forces applied on its boundaries, parallel to the plane of the plate, this state is called plane stress, whereas when the dimensions of the same body is very large when compared to the other two dimensions, this is termed plane strain. The plane states are 
particular cases of the 3D theory of elasticity, when some assumptions are made. Thus, the three equilibrium equations for the 3D solid are the starting point of the present formulation

$$
\begin{aligned}
& \frac{\partial \sigma_{x}}{\partial x}+\frac{\partial \tau_{x y}}{\partial y}+\frac{\partial \tau_{x z}}{\partial z}+f_{x}=0 \\
& \frac{\partial \tau_{x y}}{\partial x}+\frac{\partial \sigma_{y}}{\partial y}+\frac{\partial \tau_{y z}}{\partial z}+f_{y}=0 \\
& \frac{\partial \tau_{x z}}{\partial x}+\frac{\partial \tau_{y z}}{\partial y}+\frac{\partial \sigma_{z}}{\partial z}+f_{z}=0
\end{aligned}
$$

For the plane case, it can be assumed that the stress components do not depend on $z(\partial / \partial z=0)$. For this reason, the remaining components do not vary through the thickness and they are functions of the in-plane Cartesian coordinates only $\sigma_{x}(x, y), \sigma_{y}(x, y), \tau_{x y}(x, y)$. Considering all the previous assumptions the equilibrium equations (1) become

$$
\begin{aligned}
& \frac{\partial \sigma_{x}}{\partial x}+\frac{\partial \tau_{x y}}{\partial y}+f_{x}=0 \\
& \frac{\partial \tau_{x y}}{\partial x}+\frac{\partial \sigma_{y}}{\partial y}+f_{y}=0
\end{aligned}
$$

\subsection{Plane strain state}

Consider a prismatic solid in which the plane state occurs in a plane parallel to the $x-y$ one. Hence, according to the state plane hypotheses, the not zero strain components are $\varepsilon_{x}(x, y), \varepsilon_{y}(x, y), \gamma_{x y}(x, y)$, since by definition $w=0, \partial u / \partial z=0$ and $\partial v / \partial z=0$, where $u, v, w$ are the displacements along the three Cartesian axes respectively. The well-known kinematic relations are

$$
\varepsilon_{x}=\frac{\partial u_{x}}{\partial x}, \quad \varepsilon_{y}=\frac{\partial u_{y}}{\partial y}, \quad \gamma_{x y}=\frac{\partial u_{x}}{\partial y}+\frac{\partial u_{y}}{\partial x}
$$

The stress components can be found by introducing the inverse Hooke's laws

$$
\begin{aligned}
& \sigma_{x}=(2 G+\lambda) \varepsilon_{x}+\lambda \varepsilon_{y}, \quad \sigma_{y}=\lambda \varepsilon_{x}+(2 G+\lambda) \varepsilon_{y}, \\
& \tau_{x y}=G \gamma_{x y}, \quad \sigma_{z}=\lambda \varepsilon_{x}+\lambda \varepsilon_{y}
\end{aligned}
$$

It should be pointed out that in equation (4) the normal stress $\sigma_{z}$ is not negligible. Thus, a plane strain is not also a plane stress. The elastic constants $G, \lambda$ in equation ((4)) are the shear modulus and Lamé constant. These constants are related to the better known elastic (Young's) modulus and Poisson's ratio with the following relations

$$
E=\frac{G(3 \lambda+2 G)}{\lambda+G}, \quad v=\frac{\lambda}{2(\lambda+G)}
$$

Introducing the kinematic expressions (3) into the inverse Hooke's laws (4), it is obtained (excluding the definition for the normal stress)

$$
\begin{aligned}
& \sigma_{x}=(2 G+\lambda) \frac{\partial u_{x}}{\partial x}+\lambda \frac{\partial u_{y}}{\partial y}, \\
& \sigma_{y}=\lambda \frac{\partial u_{x}}{\partial x}+(2 G+\lambda) \frac{\partial u_{y}}{\partial y}, \\
& \tau_{x y}=G\left(\frac{\partial u_{x}}{\partial y}+\frac{\partial u_{y}}{\partial x}\right)
\end{aligned}
$$

Finally, the governing equations in terms of displacements can be found using (6) into (2) as follows

$$
\begin{aligned}
& (2 G+\lambda) \frac{\partial^{2} u}{\partial x^{2}}+G \frac{\partial^{2} u}{\partial y^{2}}+(\lambda+G) \frac{\partial^{2} v}{\partial x \partial y}+f_{x}=0 \\
& (\lambda+G) \frac{\partial^{2} u}{\partial x \partial y}+G \frac{\partial^{2} v}{\partial x^{2}}+(2 G+\lambda) \frac{\partial^{2} v}{\partial y^{2}}+f_{y}=0
\end{aligned}
$$

Equations (7) correspond to the elastostatic case for the plane strain case. However, if the elastodynamic problem has to be solved, the inertia forces should be added to the governing equations as

$$
\begin{aligned}
& (2 G+\lambda) \frac{\partial^{2} u}{\partial x^{2}}+G \frac{\partial^{2} u}{\partial y^{2}}+(\lambda+G) \frac{\partial^{2} v}{\partial x \partial y}+f_{x}=\rho \frac{\partial^{2} u}{\partial t^{2}} \\
& (\lambda+G) \frac{\partial^{2} u}{\partial x \partial y}+G \frac{\partial^{2} v}{\partial x^{2}}+(2 G+\lambda) \frac{\partial^{2} v}{\partial y^{2}}+f_{y}=\rho \frac{\partial^{2} v}{\partial t^{2}}
\end{aligned}
$$

where $\rho$ represents the material density. If the free vibration problem is wanted to be studied, the body forces should be set zero $f_{x}=f_{y}=0$. In the present paper the free vibration problem is investigated, thus the dynamic solution is found in the form

$$
\begin{aligned}
& u(x, y, t)=\Phi_{x}(x, y) e^{i \omega t} \\
& v(x, y, t)=\Phi_{y}(x, y) e^{i \omega t}
\end{aligned}
$$

Substituting equation (9) into equation (8) one yields

$$
\begin{aligned}
& (2 G+\lambda) \frac{\partial^{2} \Phi_{x}}{\partial x^{2}}+G \frac{\partial^{2} \Phi_{x}}{\partial y^{2}}+(\lambda+G) \frac{\partial^{2} \Phi_{y}}{\partial x \partial y}+ \\
& +\rho \omega^{2} \Phi_{x} u=0 \\
& (\lambda+G) \frac{\partial^{2} \Phi_{x}}{\partial x \partial y}+G \frac{\partial^{2} \Phi_{y}}{\partial x^{2}}+(2 G+\lambda) \frac{\partial^{2} \Phi_{y}}{\partial y^{2}}+ \\
& +\rho \omega^{2} \Phi_{y} v=0
\end{aligned}
$$

where $\Phi_{x}, \Phi_{y}$ are the mode shapes related to the in-plane displacements $u, v$ and $\omega$ represents the natural circular frequencies of the structure under study.

\subsection{Plane stress state}

In order to solve the plane stress problem, it is possible to follow the same mathematical developments of the strain case above. Thus the same systems of equations (7) and (8) can be found substituting $\lambda \rightarrow \lambda^{\star}$ where $\lambda^{\star}=\frac{2 G \lambda}{2 G+\lambda}$ or $\lambda^{\star}=$ $\frac{E v}{1-v^{2}}$. In the following all the equations are reported using $G, \lambda$ for the strain case, taking into account that the stress case can be found using $G, \lambda^{\star}$. 


\subsection{Boundary conditions}

In order to solve any partial differential system of equations, boundary conditions must be introduced. According to the theory of elasticity [1-3] the boundary conditions for a 3D solid considering the state plane assumptions are

$$
\begin{aligned}
& \sigma_{x} n_{x}+\tau_{x y} n_{y}+p_{x}=0 \\
& \tau_{x y} n_{x}+\sigma_{y} n_{y}+p_{y}=0
\end{aligned}
$$

where $n_{x}, n_{y}$ are the direction cosines of the outward unit normal of the current edge, written with respect to the outer Cartesian reference system. However, it happens that the edge is generally oriented, so that a transformation matrix should be applied to the stress components in order to evaluate the normal and tangential stresses of that edge. Furthermore, the same occurs for the in-plane displacement parameters. For these reasons the following relations can be written according to the literature [1-3]

$$
\begin{aligned}
& u_{n}=u n_{x}+v n_{y} \\
& u_{t}=v n_{x}-u n_{y} \\
& \sigma_{n}=\sigma_{x} n_{x}^{2}+\sigma_{y} n_{y}^{2}+2 \tau_{x y} n_{x} n_{y} \\
& \tau_{n t}=\left(\sigma_{y}-\sigma_{x}\right) n_{x} n_{y}+\tau_{x y}\left(n_{x}^{2}-n_{y}^{2}\right)
\end{aligned}
$$

where $u_{n}, u_{t}$ are the normal and tangential displacements and $\sigma_{n}, \tau_{n t}$ are the normal and shear stresses at the edge. For the application of the SFEM all the equations should be written as functions of the displacement parameters as in the following

$$
\begin{aligned}
u_{n}= & u n_{x}+v n_{y}, \quad u_{t}=v n_{x}-u n_{y} \\
\sigma_{n}= & \left((2 G+\lambda) n_{x}^{2}+\lambda n_{y}^{2}\right) \frac{\partial u}{\partial x}+2 G n_{x} n_{y} \frac{\partial u}{\partial y}+ \\
& +2 G n_{x} n_{y} \frac{\partial v}{\partial x}+\left((2 G+\lambda) n_{y}^{2}+\lambda n_{x}^{2}\right) \frac{\partial v}{\partial y} \\
\tau_{n t}= & -2 G n_{x} n_{y} \frac{\partial u}{\partial x}+G\left(n_{x}^{2}-n_{y}^{2}\right) \frac{\partial u}{\partial y}+ \\
& +G\left(n_{x}^{2}-n_{y}^{2}\right) \frac{\partial v}{\partial x}+2 G n_{x} n_{y} \frac{\partial v}{\partial y}
\end{aligned}
$$

\section{Discretized forms}

It is recalled that the SFEM is based on the DQ method which discretizes the derivative of a function as a weighted linear sum of some functional values. The main advantage of the DQ method is the possibility of having extremely high accuracy. However, its principal drawback is that it cannot deal with arbitrarily shaped domains and cannot have discontinuities. In order to overcome these difficulties a domain decomposition approach is followed. Thus, the whole problem is numerically solved in several domains or elements, which compose the global geometry. Subsequently, all these elements are connected among them in order to achieve the global solution. Hence, the governing equations are discretized inside each element, whereas continuity conditions are used in order to connect all of them. In the present section the discrete form of all these equations are presented in order to give a general overview on the problem. The 2D DQ implementation has been already presented by several authors in literature [33-84]. Summarizing, the DQ method can approximate a derivative along a direction using several grid points located along two directions in the reference coordinate system. However, the present approach is general and the DQ method is applied at the master element level as in the standard FEM where the Gauss quadrature is applied in the master element. For this reason all the following formulae are referred to the local master element reference system $\xi-\eta$. At the end it will be shown how to solve the differential problems in Cartesian coordinates (7) and (8) using the following notation.

\subsection{Derivative approximation and mapping}

As any other collocation method, in order to accurately approximate the derivative, two grid locations have to be set, one along $\xi$ and the other along $\eta$. It is recalled that the reference element or master element or parent element belongs to the unit space as already presented in the previous works [126-135]. The number of points, which defines these collocations, is indicated by $N$ and $M$ respectively. The weighting coefficients can be evaluated afterwards using the classic formulae provided by the DQ and the GDQ approaches. In this way two matrices, containing the weighting coefficients of all the points of the master element, are carried out. So that, the following derivatives can be approximated

$$
\begin{aligned}
& \left.\frac{\partial^{(n)} f(\xi, \eta)}{\partial \xi^{(n)}}\right|_{\substack{\xi=\xi_{i} \\
\eta=\eta_{j}}}=\sum_{k=1}^{N} \zeta_{i, k}^{\xi(n)} f_{k, j}= \\
& =\varsigma_{i, 1}^{\xi(n)} f_{1, j}+\zeta_{i, 2}^{\xi(n)} f_{2, j}+\cdots+\varsigma_{i N}^{\xi(n)} f_{N, j} \\
& \left.\frac{\partial^{(m)} f(\xi, \eta)}{\partial \eta^{(m)}}\right|_{\substack{\xi=\xi_{i} \\
\eta=\eta_{j}}}=\sum_{l=1}^{M} \varsigma_{j, l}^{\eta(m)} f_{i, l}= \\
& =\varsigma_{j, 1}^{\eta(m)} f_{i, 1}+\varsigma_{j, 2}^{\eta(m)} f_{i, 2}+\cdots+\varsigma_{j, M}^{\eta(m)} f_{i, M} \\
& \left.\frac{\partial^{(n+m)} f(\xi, \eta)}{\partial \xi^{(n)} \partial \eta^{(m)}}\right|_{\substack{\xi=\xi_{i} \\
\eta=\eta_{j}}}=\sum_{k=1}^{N} \varsigma_{i, k}^{\xi(n)}\left(\sum_{l=1}^{M} \varsigma_{j, l}^{\eta(m)} f_{k, l}\right)= \\
& =\varsigma_{i, 1}^{\xi(n)}\left(\varsigma_{j, 1}^{\eta(m)} f_{1,1}+\varsigma_{j, 2}^{\eta(m)} f_{1,2}+\cdots+\varsigma_{j, M}^{\eta(m)} f_{1, M}\right)+ \\
& +\varsigma_{i, 2}^{\xi(n)}\left(\varsigma_{j, 1}^{\eta(m)} f_{2,1}+\varsigma_{j, 2}^{\eta(m)} f_{2,2}+\cdots+\varsigma_{j, M}^{\eta(m)} f_{2, M}\right)+ \\
& +\cdots+ \\
& ,+\varsigma_{i, N}^{\xi(n)}\left(\varsigma_{j, 1}^{\eta(m)} f_{N, 1}+\varsigma_{j, 2}^{\eta(m)} f_{N, 2}+\cdots+\varsigma_{j, M}^{\eta(m)} f_{N, M}\right)
\end{aligned}
$$

where for the sake of conciseness $f\left(\xi_{i}, \eta_{j}\right)=f_{i, j}$. It must be pointed out that $i, j$ define the location of the point at which the derivative is evaluated and $k, l$ are their respec- 
tively sum indices. Observing equation (14) the first expression takes all the points which have $j$ fixed, whereas the second one fixes $i$ index. Finally, the mixed derivative comprehends all the points of the element, since $k, l$ appear both in the third of equation (14). In order to be implemented in a computer code the expressions (14) have to be written in matrix form. The easiest and more compact way is to use the Kronecker product $\otimes$, which is an operation on two matrices of arbitrary size resulting in a block matrix. For instance $\mathbf{A} \otimes \mathbf{B}$ of size $n \times m$ and $p \times q$ respectively, the resulting product gives a block matrix of size $n p \times m q$. Hence, if $\boldsymbol{\varsigma}^{\xi(n)}$ and $\boldsymbol{\varsigma}^{\eta(n)}$ indicate the matrices of the weighting coefficients along $\xi$ and $\eta$, the resulting block matrices for all the points presented in equation (14) are given by

$$
\begin{aligned}
& \underset{N M \times N M}{\mathbf{C}^{\xi(\mathrm{n})}}=\underset{M \times M}{\mathbf{I}} \otimes \underset{N \times N}{\boldsymbol{\zeta}^{\xi(n)},} \\
& \underset{N M \times N M}{\mathbf{C}^{\eta(\mathrm{m})}}=\boldsymbol{\varsigma}_{M \times M}^{\eta(m)} \otimes \underset{N \times N}{\mathbf{I}}, \\
& \underset{N M \times N M}{\mathbf{C}^{\xi \eta(\mathrm{n}+\mathrm{m})}}=\underset{M \times M}{\boldsymbol{\zeta}^{\eta(m)} \otimes \underset{N \times N}{\boldsymbol{\zeta}^{\xi(n)}}}
\end{aligned}
$$

where $\mathbf{I}$ is the identity matrix. At this point, the derivatives of any order for the master element can be evaluated using expressions (15). In detail, each row of the matrices $\mathbf{C}^{\xi(n)}$, $\mathbf{C}^{\eta(m)}, \mathbf{C}^{\xi \eta(n+m)}$ represents the approximation of the derivative of the generic point $\xi_{i}, \eta_{j}$ of the grid. The components of the matrices (15) are indicated by $C_{k l}^{\xi(n)}, C_{k l}^{\eta(m)}, C_{k l}^{\xi \eta(n+m)}$ for $k, l=i+(j-1) N$ with $i=1,2, \ldots, N$ and $j=1,2, \ldots, M$. It is important to understand the meaning of each row of the matrices (15). With reference to Figure 1 it is clear that the grid point order is taken "by columns" such as $\left(\xi_{1}, \eta_{1}\right)$, $\left(\xi_{2}, \eta_{1}\right), \ldots,\left(\xi_{N}, \eta_{1}\right),\left(\xi_{1}, \eta_{2}\right), \ldots,\left(\xi_{N}, \eta_{2}\right), \ldots \ldots,\left(\xi_{1}, \eta_{M}\right)$, $\ldots,\left(\xi_{N}, \eta_{M}\right)$. In conclusion the whole vector can be seen as $M$ times a vector of length $N$. For the sake of simplicity another sequence should be defined since the grid location should be set in vector form for computational needs. For this reason the arrow in Figure 1 has been drawn, so that the point coordinates can be grouped in vector form as

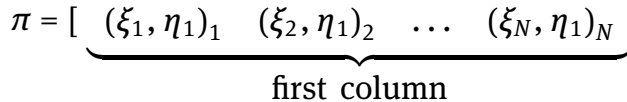

$$
\begin{aligned}
& \underbrace{\left(\xi_{1}, \eta_{2}\right)_{N+1} \quad \cdots \quad\left(\xi_{N}, \eta_{2}\right)_{2 N}} \ldots \ldots \\
& \text { second column } \\
& \underbrace{\left(\xi_{1}, \eta_{M}\right)_{N \cdot M-N+1} \quad \cdots \quad\left(\xi_{N}, \eta_{M}\right)_{N \cdot M}}_{\text {last column }}]^{T}
\end{aligned}
$$

The definition (16) can be shortened as follows

$$
\pi_{k}=\left(\xi_{i}, \eta_{j}\right)_{k} \text { for } \quad \begin{aligned}
& i=1,2, \ldots, N \\
& j=1,2, \ldots, M \\
& k
\end{aligned}
$$

In conclusion $\pi_{k}$ defines the location of all the points in the master element.

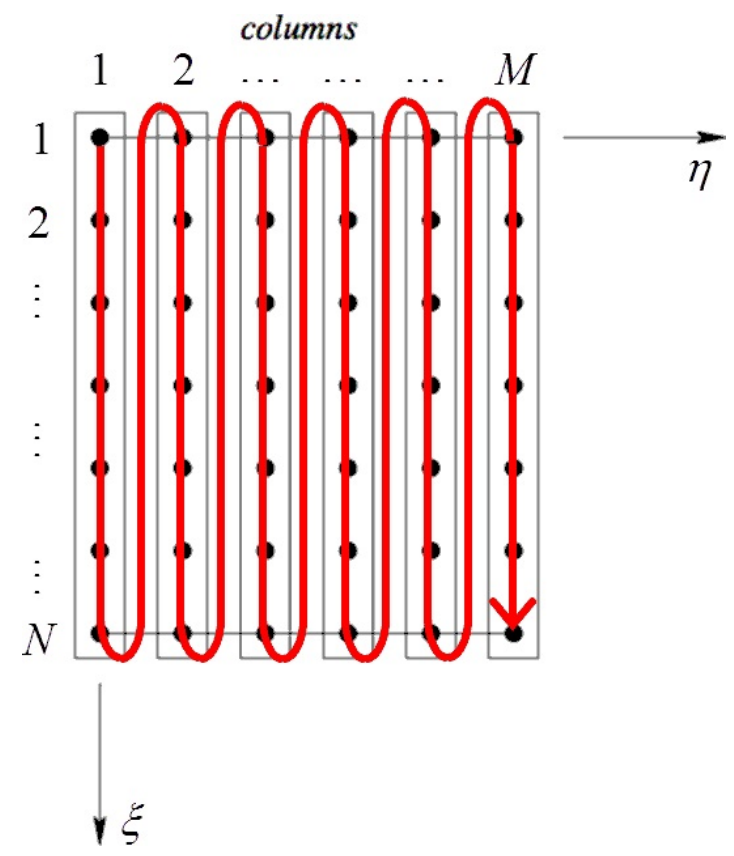

Figure 1: Grid point orders for DQ computation: the column order (boxes) and the corresponding vector (arrow).

The approximate derivatives (15) are related to the master element system $\xi-\eta$, nevertheless the governing equations are referred to the outer Cartesian one. Thus, the mapping technique should be introduced into equations (7) and (8) in order to investigate structures of arbitrary shape. A general presentation for a given set of order $m$ of shape functions is given by

$$
x=\sum_{i=1}^{m} P_{i}(\xi, \eta) x_{i}, \quad y=\sum_{i=1}^{m} P_{i}(\xi, \eta) y_{i}
$$

It has been already presented by previously published articles that, the first and second order derivatives for a standard linear mapping are the following

$$
\begin{aligned}
\frac{\partial}{\partial x}= & \frac{\partial}{\partial \xi} \xi_{x}+\frac{\partial}{\partial \eta} \eta_{x} \\
\frac{\partial^{2}}{\partial x^{2}}= & \xi_{x}^{2} \frac{\partial^{2}}{\partial \xi^{2}}+\eta_{x}^{2} \frac{\partial^{2}}{\partial \eta^{2}}+2 \xi_{x} \eta_{x} \frac{\partial^{2}}{\partial \xi \partial \eta}+\xi_{x x} \frac{\partial}{\partial \xi}+\eta_{x x} \frac{\partial}{\partial \eta} \\
\frac{\partial}{\partial y}= & \frac{\partial}{\partial \xi} \xi_{y}+\frac{\partial}{\partial \eta} \eta_{y} \\
\frac{\partial^{2}}{\partial y^{2}}= & \xi_{y}^{2} \frac{\partial^{2}}{\partial \xi^{2}}+\eta_{y}^{2} \frac{\partial^{2}}{\partial \eta^{2}}+2 \xi_{y} \eta_{y} \frac{\partial^{2}}{\partial \xi \partial \eta}+\xi_{y y} \frac{\partial}{\partial \xi}+\eta_{y y} \frac{\partial}{\partial \eta} \\
\frac{\partial^{2}}{\partial x \partial y}= & \xi_{x} \xi_{y} \frac{\partial^{2}}{\partial \xi^{2}}+\eta_{x} \eta_{y} \frac{\partial^{2}}{\partial \eta^{2}}+\left(\xi_{x} \eta_{y}+\xi_{y} \eta_{x}\right) \frac{\partial^{2}}{\partial \xi \partial \eta}+ \\
& +\xi_{x y} \frac{\partial}{\partial \xi}+\eta_{x y} \frac{\partial}{\partial \eta}
\end{aligned}
$$


where $\xi_{x}=\frac{\partial \xi}{\partial x}, \xi_{y}=\frac{\partial \xi}{\partial y}, \eta_{x}=\frac{\partial \eta}{\partial x}$ and $\eta_{y}=\frac{\partial \eta}{\partial y}$. These coordinate derivatives depend on the Jacobian matrix of the transformation and on the shape functions (mapping nodal coordinates) used, as follows

$$
\begin{aligned}
& \xi_{x}=\frac{y_{\eta}}{\operatorname{det} \mathbf{J}}, \quad \xi_{y}=-\frac{x_{\eta}}{\operatorname{det} \mathbf{J}}, \quad \eta_{x}=-\frac{y_{\xi}}{\operatorname{det} \mathbf{J}}, \\
& \eta_{y}=\frac{x_{\xi}}{\operatorname{det} \mathbf{J}} \quad \text { for } \quad \operatorname{det} \mathbf{J}=x_{\xi} y_{\eta}-x_{\eta} y_{\xi}
\end{aligned}
$$

where $x_{\xi}=\frac{\partial x}{\partial \xi}, x_{\eta}=\frac{\partial x}{\partial \eta}, y_{\xi}=\frac{\partial y}{\partial \xi}, y_{\eta}=\frac{\partial y}{\partial \eta}$ are the derivatives of the Cartesian coordinates of each mapped element using a given mapping of order $m$ (18). Hence, they are easily known. As far as the mapping technique is concerned, 4 node elements (linear), 8 node elements (quadratic), 12 node elements (cubic) have been presented in [126137], but the mapping could be general such as the one presented by Zhong and He [138]. Expressions (20) represent the first order derivatives for the mapping transformation. However, the second order must be computed, since they appear in equation (19). So, evaluating the derivatives of (20) accordingly, the following expressions appear

$$
\begin{aligned}
& \xi_{x x}=\frac{1}{\operatorname{det} \mathbf{J}^{2}}\left(y_{\eta} y_{\xi \eta}-\frac{y_{\eta}^{2}}{\operatorname{det} \mathbf{J}} \operatorname{det} \mathbf{J}_{\xi}-y_{\xi} y_{\eta \eta}+\frac{y_{\xi} y_{\eta}}{\operatorname{det} \mathbf{J}} \operatorname{det} \mathbf{J}_{\eta}\right) \\
& \xi_{y y}=\frac{1}{\operatorname{det} \mathbf{J}^{2}}\left(x_{\eta} x_{\xi \eta}-\frac{x_{\eta}^{2}}{\operatorname{det} \mathbf{J}} \operatorname{det} \mathbf{J}_{\xi}-x_{\xi} x_{\eta \eta}+\frac{x_{\xi} x_{\eta}}{\operatorname{det} \mathbf{J}} \operatorname{det} \mathbf{J}_{\eta}\right) \\
& \xi_{x y}=\frac{1}{\operatorname{det} \mathbf{J}^{2}}\left(-y_{\eta} x_{\xi \eta}+\frac{y_{\eta} x_{\eta}}{\operatorname{det} \mathbf{J}} \operatorname{det} \mathbf{J}_{\xi}+y_{\xi} x_{\eta \eta}-\frac{y_{\xi} x_{\eta}}{\operatorname{det} \mathbf{J}} \operatorname{det} \mathbf{J}_{\eta}\right) \\
& \eta_{x x}=\frac{1}{\operatorname{det} \mathbf{J}^{2}}\left(-y_{\eta} y_{\xi \xi}+\frac{y_{\xi} y_{\eta}}{\operatorname{det} \mathbf{J}} \operatorname{det} \mathbf{J}_{\xi}+y_{\xi} y_{\xi \eta}-\frac{y_{\xi}^{2}}{\operatorname{det} \mathbf{J}} \operatorname{det} \mathbf{J}_{\eta}\right) \\
& \eta_{y y}=\frac{1}{\operatorname{det} \mathbf{J}^{2}}\left(-x_{\eta} x_{\xi \xi}+\frac{x_{\xi} x_{\eta}}{\operatorname{det} \mathbf{J}} \operatorname{det} \mathbf{J}_{\xi}+x_{\xi} x_{\xi \eta}-\frac{x_{\xi}^{2}}{\operatorname{det} \mathbf{J}} \operatorname{det} \mathbf{J}_{\eta}\right) \\
& \eta_{x y}=\frac{1}{\operatorname{det} \mathbf{J}^{2}}\left(-y_{\xi} x_{\xi \eta}-\frac{x_{\xi} y_{\eta}}{\operatorname{det} \mathbf{J}} \operatorname{det} \mathbf{J}_{\xi}+y_{\eta} x_{\xi \xi}+\frac{y_{\xi} x_{\xi}}{\operatorname{det} \mathbf{J}} \operatorname{det} \mathbf{J}_{\eta}\right)
\end{aligned}
$$

where det $\mathbf{J}_{\xi}=x_{\xi} y_{\xi \eta}-y_{\xi} x_{\xi \eta}+y_{\eta} x_{\xi \xi}-x_{\eta} y_{\xi \xi}$ and $\operatorname{det} \mathbf{J}_{\eta}=$ $-x_{\eta} y_{\xi \eta}+y_{\eta} x_{\xi \eta}-y_{\xi} x_{\eta \eta}+x_{\xi} y_{\eta \eta}$. As a consequence the derivatives of the Cartesian coordinates take the form

$$
\begin{aligned}
& x_{\xi \xi}=\frac{\partial^{2} x}{\partial \xi^{2}}, x_{\eta \eta}=\frac{\partial^{2} x}{\partial \eta^{2}}, x_{\xi \eta}=\frac{\partial^{2} x}{\partial \xi \partial \eta}, \\
& y_{\zeta \xi}=\frac{\partial^{2} y}{\partial \xi^{2}}, \quad y_{\eta \eta}=\frac{\partial^{2} y}{\partial \eta^{2}}, y_{\xi \eta}=\frac{\partial^{2} y}{\partial \xi \partial \eta}
\end{aligned}
$$

Summarizing, by simple algebraic manipulations expressions (20) and (21) can be evaluated ones the element type is chosen ( 4 node, 8 node, 12 node, etc... ). Expressions (19) can be evaluated afterwards. The first step is to carry out the derivatives of the Cartesian coordinates with respect to the local master element system. Each point in Cartesian coordinates has its own mapping transformation. Hence, $x_{\xi \xi}, x_{\xi}, x_{\eta \eta}, x_{\eta}, x_{\xi \eta}, y_{\xi \xi}, y_{\xi}, y_{\eta \eta}, y_{\eta}, y_{\xi \eta}$ are vectors of dimension $N \cdot M \times 1$, since the whole domain has $N \cdot M$ points of coordinates. Following the nomenclature defined by expressions (16) and (17) it is possible to define the following vectors

$$
\begin{array}{lllll}
\mathbf{x}_{\xi \xi}, & \mathbf{x}_{\xi}, & \mathbf{x}_{\eta \eta}, & \mathbf{x}_{\eta}, & \mathbf{x}_{\xi \eta} \\
\mathbf{y}_{\xi \xi}, & \mathbf{y}_{\xi}, & \mathbf{y}_{\eta \eta}, & \mathbf{y}_{\eta}, & \mathbf{y}_{\xi \eta}
\end{array}
$$

and their components

$$
\begin{aligned}
& \left(x_{\xi \xi}\right)_{k}, \quad\left(x_{\xi}\right)_{k}, \quad\left(x_{\eta \eta}\right)_{k}, \quad\left(x_{\eta}\right)_{k}, \quad\left(x_{\xi \eta}\right)_{k} \\
& \left(y_{\xi \xi}\right)_{k}, \quad\left(y_{\xi}\right)_{k}, \quad\left(y_{\eta \eta}\right)_{k}, \quad\left(y_{\eta}\right)_{k}, \quad\left(y_{\xi \eta}\right)_{k} \\
& k=i+(j-1) N \\
& \text { for } \quad i=1,2, \ldots, N \\
& j=1,2, \ldots, M
\end{aligned}
$$

Now equations (20) and (21) can be evaluated. Thus, the following vectors can be numerically defined

$$
\begin{array}{llll}
\boldsymbol{\xi}_{x x}, & \boldsymbol{\xi}_{x}, \quad \boldsymbol{\xi}_{y y}, \quad \boldsymbol{\xi}_{y}, \quad \boldsymbol{\xi}_{x y} \\
\boldsymbol{\eta}_{x x}, & \boldsymbol{\eta}_{x}, \quad \boldsymbol{\eta}_{y y}, \quad \boldsymbol{\eta}_{y}, \quad \boldsymbol{\eta}_{x y} \\
\operatorname{det} \mathbf{J}= & \mathbf{x}_{\xi} \mathbf{y}_{\eta}-\mathbf{x}_{\eta} \mathbf{y}_{\xi} \\
\text { for } \quad \operatorname{det} \mathbf{J}_{\xi}= & \mathbf{x}_{\xi} \mathbf{y}_{\xi \eta}-\mathbf{y}_{\xi} \mathbf{x}_{\xi \eta}+\mathbf{y}_{\eta} \mathbf{x}_{\xi \xi}-\mathbf{x}_{\eta} \mathbf{y}_{\xi \xi} \\
& \operatorname{det} \mathbf{J}_{\eta}=-\mathbf{x}_{\eta} \mathbf{y}_{\xi \eta}+\mathbf{y}_{\eta} \mathbf{x}_{\xi \eta}-\mathbf{y}_{\xi} \mathbf{x}_{\eta \eta}+\mathbf{x}_{\xi} \mathbf{y}_{\eta \eta}
\end{array}
$$

or their components in their correspondent form

$$
\begin{aligned}
& \left(\xi_{x x}\right)_{k}, \quad\left(\xi_{x}\right)_{k}, \quad\left(\xi_{y y}\right)_{k}, \quad\left(\xi_{y}\right)_{k}, \quad\left(\xi_{x y}\right)_{k}, \\
& \left(\eta_{x x}\right)_{k}, \quad\left(\eta_{x}\right)_{k}, \quad\left(\eta_{y y}\right)_{k}, \quad\left(\eta_{y}\right)_{k}, \quad\left(\eta_{x y}\right)_{k} \\
& (\operatorname{det})_{k}=\left(x_{\xi}\right)_{k}\left(y_{\eta}\right)_{k}-\left(x_{\eta}\right)_{k}\left(y_{\xi}\right)_{k} \\
& \left(\operatorname{det} \mathbf{J}_{\xi}\right)_{k}=\left(x_{\xi}\right)_{k}\left(y_{\xi \eta}\right)_{k}-\left(y_{\xi}\right)_{k}\left(x_{\xi \eta}\right)_{k}+ \\
& \quad+\left(y_{\eta}\right)_{k}\left(x_{\xi \xi}\right)_{k}-\left(x_{\eta}\right)_{k}\left(y_{\xi \xi}\right)_{k} \\
& \left(\operatorname{det} \mathbf{J}_{\eta}\right)_{k}=-\left(x_{\eta}\right)_{k}\left(y_{\xi \eta}\right)_{k}+\left(y_{\eta}\right)_{k}\left(x_{\xi \eta}\right)_{k}+ \\
& \quad-\left(y_{\xi}\right)_{k}\left(x_{\eta \eta}\right)_{k}+\left(x_{\xi}\right)_{k}\left(y_{\eta \eta}\right)_{k} \\
& \text { for } k=i+(j-1) N \text { with } i=1,2, \ldots, N \\
& \text { and } j=1,2, \ldots, M
\end{aligned}
$$

Finally, using equations (25) and the block matrices (15) the map of the Cartesian derivatives with respect to the local reference system of the master element (19) can be carried out. Thus, equations (19) become

$$
\begin{aligned}
& D_{k l}^{x(1)}=\left(\xi_{x}\right)_{k} C_{k l}^{\xi(1)}+\left(\eta_{x}\right)_{k} C_{k l}^{\eta(1)} \\
& D_{k l}^{x(2)}=\left(\xi_{x}\right)_{k}^{2} C_{k l}^{\xi(2)}+\left(\eta_{x}\right)_{k}^{2} C_{k l}^{\eta(2)}+ \\
&+2\left(\xi_{x}\right)_{k}\left(\eta_{x}\right)_{k} C_{k l}^{\xi \eta(11)}+\left(\xi_{x x}\right)_{k} C_{k l}^{\xi(1)}+ \\
&+\left(\eta_{x x}\right)_{k} C_{k l}^{\eta(1)} \\
& D_{k l}^{y(1)}=\left(\xi_{y}\right)_{k} C_{k l}^{\xi(1)}+\left(\eta_{y}\right)_{k} C_{k l}^{\eta(1)} \\
& D_{k l}^{y(2)}=\left(\xi_{y}\right)_{k}^{2} C_{k l}^{\xi(2)}+\left(\eta_{y}\right)_{k}^{2} C_{k l}^{\eta(2)}+ \\
&+2\left(\xi_{y}\right)_{k}\left(\eta_{y}\right)_{k} C_{k l}^{\xi \eta(11)}+\left(\xi_{y y}\right)_{k} C_{k l}^{\xi(1)}+ \\
&+\left(\eta_{y y}\right)_{k} C_{k l}^{\eta(1)} \\
& D_{k l}^{x y(11)}=\left(\xi_{x}\right)_{k}\left(\xi_{y}\right)_{k} C_{k l}^{\xi(2)}+\left(\eta_{x}\right)_{k}\left(\eta_{y}\right)_{k} C_{k l}^{\eta(2)}+ \\
&+\left(\left(\xi_{x}\right)_{k}\left(\eta_{y}\right)_{k}+\left(\xi_{y}\right)_{k}\left(\eta_{x}\right)_{k}\right) C_{k l}^{\xi \eta(11)}+ \\
&+\left(\xi_{x y}\right)_{k} C_{k l}^{\xi(1)}+\left(\eta_{x y}\right)_{k} C_{k l}^{\eta(1)} \\
& \text { for } \quad k, l=i+(j-1) N \text { with } \quad i=1,2, \ldots, N \\
& \text { and } \quad j=1,2, \ldots, M
\end{aligned}
$$


where the components of the Cartesian derivatives $D_{k l}^{x(1)}, D_{k l}^{x(2)}, D_{k l}^{y(1)}, D_{k l}^{y(2)}, D_{k l}^{x y(11)}$ can be written in matrix form as

$$
\mathbf{D}^{x(1)}, \quad \mathbf{D}^{x(2)}, \quad \mathbf{D}^{y(1)}, \quad \mathbf{D}^{y(2)}, \quad \mathbf{D}^{x y(11)}
$$

At this point, it is possible to approximate the Cartesian derivatives using the matrices (28) which have the mapping transformation included.

Finally, the mathematical expressions for the outward unit normal vector $\mathbf{n}$ are given for the master element. Considering Figure 2 as a reference for the nomenclature of corners (numbers in circles) and edges (numbers in squares), for the first and third edges $\xi=\mp 1$ the outward unit normal vector components are

$$
\left[\begin{array}{l}
n_{x} \\
n_{y}
\end{array}\right]=\frac{\xi}{\sqrt{x_{\eta}^{2}+y_{\eta}^{2}}}\left[\begin{array}{l}
y_{\eta} \\
-x_{\eta}
\end{array}\right]
$$

whereas for the second and fourth edges $\eta= \pm 1$ they are

$$
\left[\begin{array}{l}
n_{x} \\
n_{y}
\end{array}\right]=\frac{\eta}{\sqrt{x_{\xi}^{2}+y_{\xi}^{2}}}\left[\begin{array}{c}
-y_{\xi} \\
x_{\xi}
\end{array}\right]
$$

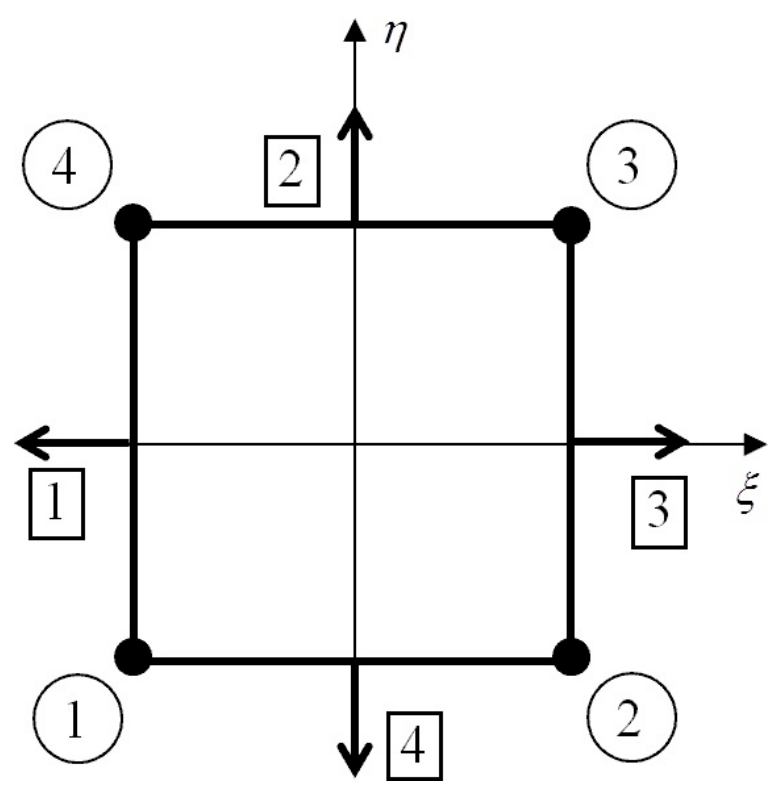

Figure 2: Corners and edges enumeration of the master element.

\subsection{Discretized form of the governing equations}

In order to write and understand the discrete forms of the elastostatic (7) and elastodynamic (8) problems the dis- placement vector $\mathbf{U}$ should be firstly defined. It can be divided into $\mathbf{U}=\left[\begin{array}{ll}\mathbf{U}_{x} & \mathbf{U}_{y}\end{array}\right]^{T}$, where $\mathbf{U}_{x}$ and $\mathbf{U}_{y}$ contain the $u$ and $v$ displacement components of all the grid points, respectively. It is remarked that $\mathbf{U}_{x}$ and $\mathbf{U}_{y}$ have dimension $N \cdot M \times 1$ and they follow the same structure indicated by expression (16). Ultimately, the single components of the displacement vectors can be indicated by $\left(U_{x}\right)_{k}$ and $\left(U_{y}\right)_{k}$ as suggested in expression (16) by $\pi_{k}$. Thus, using the definition of the derivation matrices (27) and the governing equations of the elastostatic problem (7) the following discrete form is exhibited

$$
\begin{aligned}
& (2 G+\lambda) \sum_{l=1}^{N \cdot M} D_{k l}^{x(2)}\left(U_{x}\right)_{l}+G \sum_{l=1}^{N \cdot M} D_{k l}^{y(2)}\left(U_{x}\right)_{l}+ \\
& +(\lambda+G) \sum_{l=1}^{N \cdot M} D_{k l}^{x y(11)}\left(U_{y}\right)_{l}+\left(F_{x}\right)_{k}=0 \\
& (\lambda+G) \sum_{l=1}^{N \cdot M} D_{k l}^{x y(11)}\left(U_{x}\right)_{l}+G \sum_{l=1}^{N \cdot M} D_{k l}^{x(2)}\left(U_{y}\right)_{l}+ \\
& +(2 G+\lambda) \sum_{l=1}^{N \cdot M} D_{k l}^{y(2)}\left(U_{y}\right)_{l}+\left(F_{y}\right)_{k}=0 \\
& \text { for } \quad k=i+(j-1) N \quad \text { with } i=1,2, \ldots, N \\
& \text { and } \quad j=1,2, \ldots, M
\end{aligned}
$$

where $\left(F_{x}\right)_{k},\left(F_{y}\right)_{k}$ are the components of the force vectors $\mathbf{F}_{x}, \mathbf{F}_{y}$ which obviously have the same structures indicated by equation (16). In the present case $G$ and $\lambda$ are constant terms. Hence, the present implementation allows to consider mechanical properties that vary element by element. It is remarked that equation (31) is written at the master element level, since the mapping technique is embedded in the derivative terms. A compact form of equation (31) is given in the following

$$
\begin{aligned}
& (2 G+\lambda) \mathbf{D}^{x(2)} \mathbf{U}_{x}+G \mathbf{D}^{y(2)} \mathbf{U}_{x}+(\lambda+G) \mathbf{D}^{x y(11)} \mathbf{U}_{y}+\mathbf{F}_{x}=\mathbf{0} \\
& (\lambda+G) \mathbf{D}^{x y(11)} \mathbf{U}_{x}+G \mathbf{D}^{x(2)} \mathbf{U}_{y}+(2 G+\lambda) \mathbf{D}^{y(2)} \mathbf{U}_{y}+\mathbf{F}_{y}=\mathbf{0}
\end{aligned}
$$

Rebuilding the displacement vector $\mathbf{U}$ equation ((32)) becomes

$$
\begin{aligned}
& {\left[\begin{array}{ll}
(2 G+\lambda) \mathbf{D}^{x(2)}+G \mathbf{D}^{y(2)} & (\lambda+G) \mathbf{D}^{x y(11)} \\
(\lambda+G) \mathbf{D}^{x y(11)} & G \mathbf{D}^{x(2)}+(2 G+\lambda) \mathbf{D}^{y(2)}
\end{array}\right]\left[\begin{array}{l}
\mathbf{U}_{x} \\
\mathbf{U}_{y}
\end{array}\right]+} \\
& +\left[\begin{array}{l}
\mathbf{F}_{x} \\
\mathbf{F}_{y}
\end{array}\right]=\left[\begin{array}{l}
\mathbf{0} \\
\mathbf{0}
\end{array}\right]
\end{aligned}
$$

It is clear that the algebraic system has dimension $(2 N \cdot M) \times(2 N \cdot M)$. Furthermore equation (33) could not be solved directly since the boundary conditions have not been applied yet to the problem. Thus, the lines related to the boundary conditions have to be substituted by the algebraic expressions of the boundary conditions. 
Using the same notation as the one presented above, equation (13) can be written in algebraic form as follows

$$
\begin{aligned}
& \left(U_{n}\right)_{k}=\left(n_{x}\right)_{k}\left(U_{x}\right)_{k}+\left(n_{y}\right)_{k}\left(U_{y}\right)_{k}, \\
& \left(U_{t}\right)_{k}=\left(n_{x}\right)_{k}\left(U_{y}\right)_{k}-\left(n_{y}\right)_{k}\left(U_{x}\right)_{k} \\
& \left(\sigma_{n}\right)_{k}=\left((2 G+\lambda)\left(n_{x}\right)_{k}^{2}+\right. \\
& \left.+\lambda\left(n_{y}\right)_{k}^{2}\right) \sum_{l=1}^{N \cdot M} D_{k l}^{x(1)}\left(U_{x}\right)_{l}+ \\
& +2 G\left(n_{x}\right)_{k}\left(n_{y}\right)_{k} \sum_{l=1}^{N \cdot M} D_{k l}^{y(1)}\left(U_{x}\right)_{l}+ \\
& +2 G\left(n_{x}\right)_{k}\left(n_{y}\right)_{k} \sum_{l=1}^{N \cdot M} D_{k l}^{x(1)}\left(U_{y}\right)_{l}+ \\
& +\left((2 G+\lambda)\left(n_{y}\right)_{k}^{2}+\lambda\left(n_{x}\right)_{k}^{2}\right) \sum_{l=1}^{N \cdot M} D_{k l}^{y(1)}\left(U_{y}\right)_{l} \\
& \left(\tau_{n t}\right)_{k}=-2 G\left(n_{x}\right)_{k}\left(n_{y}\right)_{k} \sum_{l=1}^{N \cdot M} D_{k l}^{x(1)}\left(U_{x}\right)_{l}+ \\
& +G\left(\left(n_{x}\right)_{k}^{2}-\left(n_{y}\right)_{k}^{2}\right) \sum_{l=1}^{N \cdot M} D_{k l}^{y(1)}\left(U_{x}\right)_{l}+ \\
& +G\left(\left(n_{x}\right)_{k}^{2}-\left(n_{y}\right)_{k}^{2}\right) \sum_{l=1}^{N \cdot M} D_{k l}^{x(1)}\left(U_{y}\right)_{l}+ \\
& +2 G\left(n_{x}\right)_{k}\left(n_{y}\right)_{k} \sum_{l=1}^{N \cdot M} D_{k l}^{y(1)}\left(U_{y}\right)_{l} \\
& \text { for } k=i+(j-1) N \text { with } i=1,2, \ldots, N \\
& \text { and } j=1,2, \ldots, M
\end{aligned}
$$

where $\left(n_{x}\right)_{k},\left(n_{y}\right)_{k}$ are the components of the normal vector projected along $x$ and $y$, respectively. It should be noted that equation (34) does not have to be necessarily evaluated in all the points of the domain, as indicated by the index $k$. On the contrary, only the boundary points are involved in this process. Nonetheless, in order to use the definitions (28) it is preferable to implement equation (34) skipping the points that are not involved by the boundaries. For this reason a set of algebraic equations are written for the boundary conditions in a similar form to the one presented by equation (33). This set is presented in matrix form below

$$
\begin{aligned}
\mathbf{U}_{n}= & \mathbf{n}_{x} \mathbf{U}_{x}+\mathbf{n}_{y} \mathbf{U}_{y} \\
\mathbf{U}_{t}= & \mathbf{n}_{x} \mathbf{U}_{y}-\mathbf{n}_{y} \mathbf{U}_{x} \\
\boldsymbol{\sigma}_{n}= & \left((2 G+\lambda) \mathbf{n}_{x}^{2}+\lambda \mathbf{n}_{y}^{2}\right) \mathbf{D}^{x(1)} \mathbf{U}_{x}+ \\
& +2 G \mathbf{n}_{x} \mathbf{n}_{y} \mathbf{D}^{y(1)} \mathbf{U}_{x}+2 G \mathbf{n}_{x} \mathbf{n}_{y} \mathbf{D}^{x(1)} \mathbf{U}_{y+} \\
& +\left((2 G+\lambda) \mathbf{n}_{y}^{2}+\lambda \mathbf{n}_{x}^{2}\right) \mathbf{D}^{y(1)} \mathbf{U}_{y} \\
\boldsymbol{\tau}_{n t}= & -2 G \mathbf{n}_{x} \mathbf{n}_{y} \mathbf{D}^{x(1)} \mathbf{U}_{x}+G\left(\mathbf{n}_{x}^{2}-\mathbf{n}_{y}^{2}\right) \mathbf{D}^{y(1)} \mathbf{U}_{x+} \\
& +G\left(\mathbf{n}_{x}^{2}-\mathbf{n}_{y}^{2}\right) \mathbf{D}^{x(1)} \mathbf{U}_{y}+2 G \mathbf{n}_{x} \mathbf{n}_{y} \mathbf{D}^{y(1)} \mathbf{U}_{y}
\end{aligned}
$$

Thus the kinematic displacements of (35) can be written as

$$
\begin{aligned}
& \mathbf{U}_{n}=\left[\begin{array}{ll}
\mathbf{n}_{x} & \mathbf{n}_{y}
\end{array}\right]\left[\begin{array}{l}
\mathbf{U}_{x} \\
\mathbf{U}_{y}
\end{array}\right] \\
& \mathbf{U}_{t}=\left[\begin{array}{ll}
-\mathbf{n}_{y} & \mathbf{n}_{x}
\end{array}\right]\left[\begin{array}{l}
\mathbf{U}_{x} \\
\mathbf{U}_{y}
\end{array}\right]
\end{aligned}
$$

and the stresses from (35) become

$$
\begin{aligned}
& \boldsymbol{\sigma}_{n}= \\
& {\left[\begin{array}{c}
\left((2 G+\lambda) \mathbf{n}_{x}^{2}+\lambda \mathbf{n}_{y}^{2}\right) \mathbf{D}^{x(1)}+2 G \mathbf{n}_{x} \mathbf{n}_{y} \mathbf{D}^{y(1)} \ldots \\
\ldots 2 G \mathbf{n}_{x} \mathbf{n}_{y} \mathbf{D}^{x(1)}+\left((2 G+\lambda) \mathbf{n}_{y}^{2}+\lambda \mathbf{n}_{x}^{2}\right) \mathbf{D}^{y(1)}
\end{array}\right]\left[\begin{array}{l}
\mathbf{U}_{x} \\
\mathbf{U}_{y}
\end{array}\right]} \\
& \boldsymbol{\tau}_{n t}=\left[\begin{array}{l}
-2 G \mathbf{n}_{x} \mathbf{n}_{y} \mathbf{D}^{x(1)}+G\left(\mathbf{n}_{x}^{2}-\mathbf{n}_{y}^{2}\right) \mathbf{D}^{y(1)} \ldots \\
\ldots G\left(\mathbf{n}_{x}^{2}-\mathbf{n}_{y}^{2}\right) \mathbf{D}^{x(1)}+2 G \mathbf{n}_{x} \mathbf{n}_{y} \mathbf{D}^{y(1)}
\end{array}\right]\left[\begin{array}{c}
\mathbf{U}_{x} \\
\mathbf{U}_{y}
\end{array}\right]
\end{aligned}
$$

For the sake of clarity the points that belong to the boundaries are identified by $k=1,2, \ldots, N$ (first column) $k=N+1,2 N, 2 N+1,3 N, \ldots,(M-2) N+1,(M-1) N$ (first and last points of the middle columns) and $k=$ $(M-1) N+1, \ldots, M N$ (last column). These points are part of the boundary matrices which compose the global stiffness matrix. It should be remarked that four of this set of points are the corner points, which need particular conditions in order to have a correct implementation of the method. In order to have a general implementation of the present method it is fundamental to separate the corner points from the boundary points of the edges (as suggested by Francesco Tornabene during a private communication with the author in April 2012). An analytical way of solving the corner point problem has not been given yet, even though several numerical solutions [126-137] have been proposed. Expressions (37) give all the possible combinations in order to have different boundary conditions for the plane problems. For example one can have the clamped condition $\mathbf{U}_{n}=\overline{\mathbf{U}}_{n}, \mathbf{U}_{t}=\overline{\mathbf{U}}_{t}$, the free condition $\boldsymbol{\sigma}_{n}=$ $\overline{\boldsymbol{\sigma}}_{n}, \boldsymbol{\tau}_{n t}=\overline{\boldsymbol{\tau}}_{n t}$ and the mixed condition that can be a symmetric condition respect to an axis orthogonal to the normal one $\mathbf{U}_{n}=\overline{\mathbf{U}}_{n}, \boldsymbol{\tau}_{n t}=\overline{\boldsymbol{\tau}}_{n t} . \overline{\mathbf{U}}_{n}, \overline{\mathbf{U}}_{t}$ and $\overline{\boldsymbol{\sigma}}_{n}, \overline{\boldsymbol{\tau}}_{n t}$ are the applied displacements and forces on the boundaries, respectively. Each element, of the domain decomposition, is identified by a set of domain and boundary equations. Only the domain points are taken from equation (33) and only the boundary points are extracted from equation (37). Moreover, it is more convenient to separate the boundary and domain degrees of freedom from the displacement vectors $\mathbf{U}_{x}, \mathbf{U}_{y}$. The displacement vector containing the boundary points is identified by $\mathbf{U}_{b}$ so that it has dimension $(2 N+2(M-2)) n_{d}$, where $n_{d}=2$ is the number of degrees of freedom (two in-plane displacements). Grouping the displacements of the domain points the vector $\mathbf{U}_{d}$ is defined and it has dimension $((N-2)(M-2)) n_{d}$. As it was anticipated in the introduction prior versions of the present formulations were called multi-domain differential quadrature, since regular (no mapping) divisions were employed. For these cases only $N \neq M$, nevertheless, it is possible to simplify the presentation of the whole theory considering $N=M$. Hence, the boundary points be- 
come $(4 N-4) n_{d}$ and the domain points are $(N-2)^{2} n_{d}$. The general implementation $(N \neq M)$ is recommended in order to have both techniques within the same code. In conclusion the algebraic equations for a single SFEM element are the following

$$
\left[\begin{array}{ll}
\mathbf{K}_{b b} & \mathbf{K}_{b d} \\
\mathbf{K}_{d b} & \mathbf{K}_{d d}
\end{array}\right]^{(e)}\left[\begin{array}{l}
\mathbf{U}_{b} \\
\mathbf{U}_{d}
\end{array}\right]^{(e)}+\left[\begin{array}{l}
\mathbf{F}_{b} \\
\mathbf{F}_{d}
\end{array}\right]^{(e)}=\left[\begin{array}{l}
\mathbf{0} \\
\mathbf{0}
\end{array}\right]^{(e)}
$$

where ${ }^{(e)}$ identifies the generic $\boldsymbol{e}$-th element, $\mathbf{F}_{d}$ is the vector of the domain loads $\mathbf{F}_{x}, \mathbf{F}_{y}$ and $\mathbf{F}_{b}$ is the vector of the boundary loads (that can be displacements or stresses). As far as the free vibration problem is concerned (10), its discrete form can be presented in matrix form (using the previously presented elastostatic problem (33)) as follows

$$
\begin{aligned}
& {\left[\begin{array}{ll}
(2 G+\lambda) \mathbf{D}^{x(2)}+G \mathbf{D}^{y(2)} & (\lambda+G) \mathbf{D}^{x y(11)} \\
(\lambda+G) \mathbf{D}^{x y(11)} & G \mathbf{D}^{x(2)}(2 G+\lambda) \mathbf{D}^{y(2)}
\end{array}\right]\left[\begin{array}{l}
\mathbf{U}_{x} \\
\mathbf{U}_{y}
\end{array}\right]+} \\
& +\rho \omega^{2}\left[\begin{array}{ll}
\mathbf{I} & \mathbf{0} \\
\mathbf{0} & \mathbf{I}
\end{array}\right]\left[\begin{array}{l}
\mathbf{U}_{x} \\
\mathbf{U}_{y}
\end{array}\right]=\left[\begin{array}{l}
\mathbf{0} \\
\mathbf{0}
\end{array}\right]
\end{aligned}
$$

where $\mathbf{U}_{x}, \mathbf{U}_{y}$ contain the displacement components of the mode shapes according to $\Phi_{x}, \Phi_{y}$ defined by equation (9) and Iis the identity matrix of dimension $(N \cdot M) \times(N \cdot M)$. Applying the boundary conditions (37) the final matrix form of the free vibration problem, for a generic $e$-th element, becomes

$$
\begin{aligned}
& \left(\left[\begin{array}{ll}
\mathbf{K}_{b b} & \mathbf{K}_{b d} \\
\mathbf{K}_{d b} & \mathbf{K}_{d d}
\end{array}\right]^{(e)}+\omega^{2}\left[\begin{array}{ll}
\mathbf{0} & \mathbf{0} \\
\mathbf{0} & \mathbf{M}_{d d}
\end{array}\right]^{(e)}\right)\left[\begin{array}{l}
\mathbf{U}_{b} \\
\mathbf{U}_{d}
\end{array}\right]^{(e)}= \\
& =\left[\begin{array}{l}
\mathbf{0} \\
\mathbf{0}
\end{array}\right]^{(e)}
\end{aligned}
$$

that after the assembly section can be clearly solved as an eigenvalue problem.

\subsection{Graphical representation of a SFEM mesh}

Before jumping into the set of equations for the continuity and external boundary conditions, it could be helpful to have a graphical representation of a general SFEM mesh as the one in Figure 3. This figure represents the inter-element edges and the external boundaries with solid lines. In addition Figure 4 presents the nomenclature of the unit vectors for the same mesh of Figure 3. Since quadrilateral elements are used, at least four normal vectors have to be defined. It should be noted that if the edge is curved the outward unit vector is not constant but changes point by
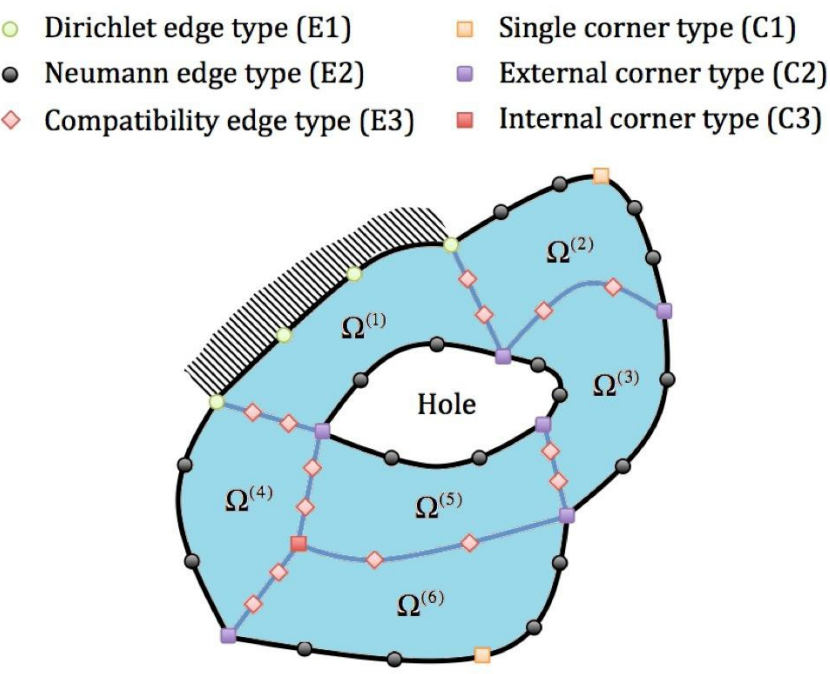

_ Inter-element edge

External boundary

Figure 3: Internal and external boundary conditions for element edges and corners.

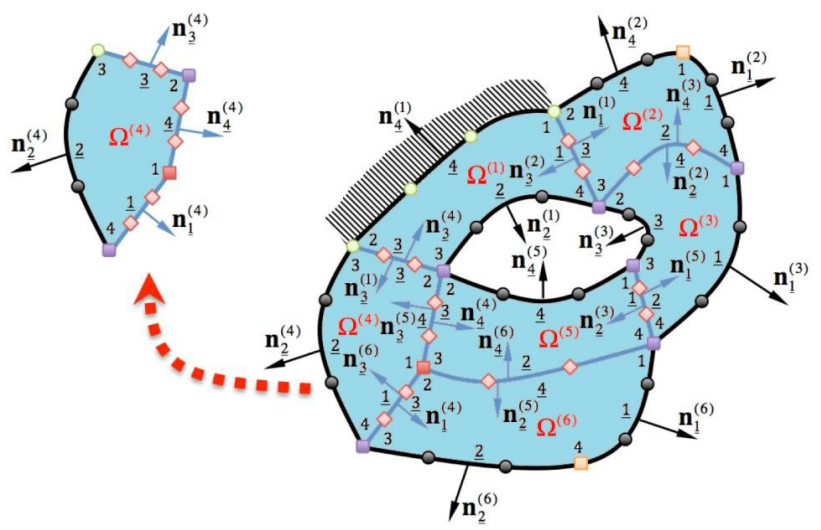

Figure 4: Outward unit normal vectors definition for a generic subdivision.

point according to the element geometry as expressed by equations (29) and (30).

It is observed that two groups of points occur, the one on the edges (E) and the others at the element corners (C).

Kinematic (Dirichlet) boundary conditions are indicated with the type E1 as for the element $\Omega^{(1)}$. Static (Neumann) boundary conditions are termed for type E2. The stress vector referred to the edge $\underline{3}$ of the element $\Omega^{(1)}$ can be indicated as $\boldsymbol{\sigma}_{\mathbf{n}_{3}}^{(1)}$. The subscript of the normal vector indicates the edge $\underline{3}$ and the superscript stands for the current element (1) that holds that normal vector. It should be emphasized that the corner conditions strongly depend on the conditions of the pair of edges at the corners where they belong. Thus, looking at the corner conditions several configurations can occur. The two corners on element 
$\Omega^{(1)}$ on the edge 4 have a kinematic (Dirichlet) condition E1 because the clamped boundary condition is stronger than a static (Neumann) one. The other corners indicated by C1 do not have static boundary conditions since two Neumann conditions have to be enforced at the same time at a single point. The compatibility conditions are indicated by E3 along the element edges. For example the edge points of element $\Omega^{(1)}$ along 1 are superimposed to the points along 3 of element $\Omega^{(2)}$. Hence, only one group of points is underlined in Figure 3. Nevertheless computationally speaking a double set of equations have to be enforced. Considering the edge $\underline{1}$ of element $\Omega^{(1)}$ and the edge $\underline{3}$ of element $\Omega^{(2)}$ that face each other, the compatibility conditions have to be enforced (the equations governing this case will be shown in the following). The external and internal corner type conditions are indicated as $\mathrm{C} 2$ and $\mathrm{C} 3$ in Figure 3. It is recalled that the corners of all the elements concurring at a specific node are superimposed as well as the points on the edges. For instance, the two corners with $\mathrm{C} 2$ conditions belong to the two neighbour elements. In the cited cases the $\mathrm{C} 2$ conditions have the same form of the E3 ones because only two elements concur at the corner. Nevertheless, the internal corners $\mathrm{C} 3$ should have different continuity conditions. The solution for that problem will be described in the following. It must be remarked that all the enforced continuity conditions are continuous with their first derivative at the interfaces, and they can be indicated as $C^{(1)}$ continuous.

\subsection{Element connectivity}

Since a strong formulation is proposed, the boundary conditions are not automatically satisfies such as in FEM. Hence, they must be defined as it was done in the previous section. However, only the external boundary conditions have been introduced. To connect the elements and performing the assemblage of the whole system, the continuity conditions should be enforced between facing elements. In order to perform that, the same definitions given by equations (37) are used. When two elements share the same boundary, two lines of points are superimposed and conditions per physical point can be written (because each grid point has 2 degrees of freedom). The continuity conditions enforce the equality of the displacements and the stresses between these two edges. Just to give an example if the element ${ }^{(e)}$ faces the element ${ }^{(e+1)}$ the following conditions must be written

$$
\begin{array}{ll}
\mathbf{U}_{n}^{(e)}-\mathbf{U}_{n}^{(e+1)}=\mathbf{0}, & \mathbf{U}_{t}^{(e)}-\mathbf{U}_{t}^{(e+1)}=\mathbf{0} \\
\boldsymbol{\sigma}_{n}^{(e)}-\boldsymbol{\sigma}_{n}^{(e+1)}=\mathbf{0}, & \boldsymbol{\tau}_{n t}^{(e)}-\boldsymbol{\tau}_{n t}^{(e+1)}=\mathbf{0}
\end{array}
$$

The algebraic equations (41) will be part of matrices $\mathbf{K}_{b b}^{(e)}$, $\mathbf{K}_{b d}^{(e, e+1)}, \mathbf{K}_{b b}^{(e+1)}, \mathbf{K}_{b d}^{(e+1, e)}$ whereas the other matrices related to the domain points are unchanged, since no mathematical condition relates the two facing elements. It can be noted that the first line of equations (41) is related to the kinematic (Dirichlet) conditions, thus no derivation is involved. Whereas the second line of equations (41) are the static (Neumann) conditions in which derivatives of the displacement parameters occur as shown by equations (37). The current implementation follows this rule: when the compatibility conditions are written between two elements, identified by ${ }^{(e)}$ and ${ }^{(e+1)}$, the kinematic conditions are enforced on the boundary points of the element (e) and the static conditions are set on the boundary points of the element ${ }^{(e+1)}$. It is recalled that when a derivative is approximated using DQ method, all the points in the derivative direction (or all the domain points in case of the mixed derivative) are involved. Hence, when the kinematic equations are considered on the boundary points of the element ${ }^{(e)}$ the matrix $\mathbf{K}_{b d}^{(e, e+1)}=\mathbf{0}$, since it contains the domains points which are related to the boundary ones. On the contrary for the static equations enforced on the boundary points of the element ${ }^{(e+1)}, \mathbf{K}_{b d}^{(e+1, e)} \neq \mathbf{0}$, since it contains the algebraic terms of the derivative approximation of the stresses between the elements. As far as the corner point conditions are concerned, their implementation could follow the approaches presented in the past [126137]. It is recalled that in order to treat the corners, different conditions should be taken into consideration as a function of the internal or external boundaries involved. The dealing of the corners is an open problem in literature and researches proposed different solutions on the subject. One of the most interesting solutions has been given by Boyd [15]: “... the corner singularities will dominate the asymptotic behaviour of the Chebyshev coefficients..., and the convergence will be algebraic rather than exponential". Moreover: "The generic recommendation is to ignore the singularities unless either (i) one has prior knowledge that $u(x, y)$ is discontinuous or has other strongly pathological behaviour or (ii) poor convergence and rapid variation of the numerical solution near the corners suggests a posteriori that the solution is strongly singular". Following the suggestions given by Boyd, for the study of the mechanics of structural components a finite element without the corner points was implemented (as suggested by Francesco Tornabene during a private communication with the author in June 2013), in order to avoid them in the program.

A graphical representation of this implementation is given in Figure 5. In order to proceed with this numerical procedure the weighting coefficients of the boundaries 
have to be computed separately from the inner points since different discretizations occur. It is remarked that this kind of implementation does not give accurate results for these structural problems and no numerical result is presented in this work. The instability of the corner-less approach is mainly due to the approximation of the mixed derivative, which is mainly involved in the free external conditions and static inter-element connectivity. Thus, the following corner point implementation has been followed. The first configuration is presented in Figure $6 \mathrm{a}$ when a corner point of a single element is studied. This corner can have two edges both clamped, both free or just one of them clamped. The symbol EB is used when external boundaries are considered, whereas the internal boundaries are indicated with IB. The represented element is the general element ${ }^{(e)}$. It is obvious that when at least one of the two edges of element ${ }^{(e)}$ is clamped the corner is fixed too. So only kinematic conditions have to be imposed.

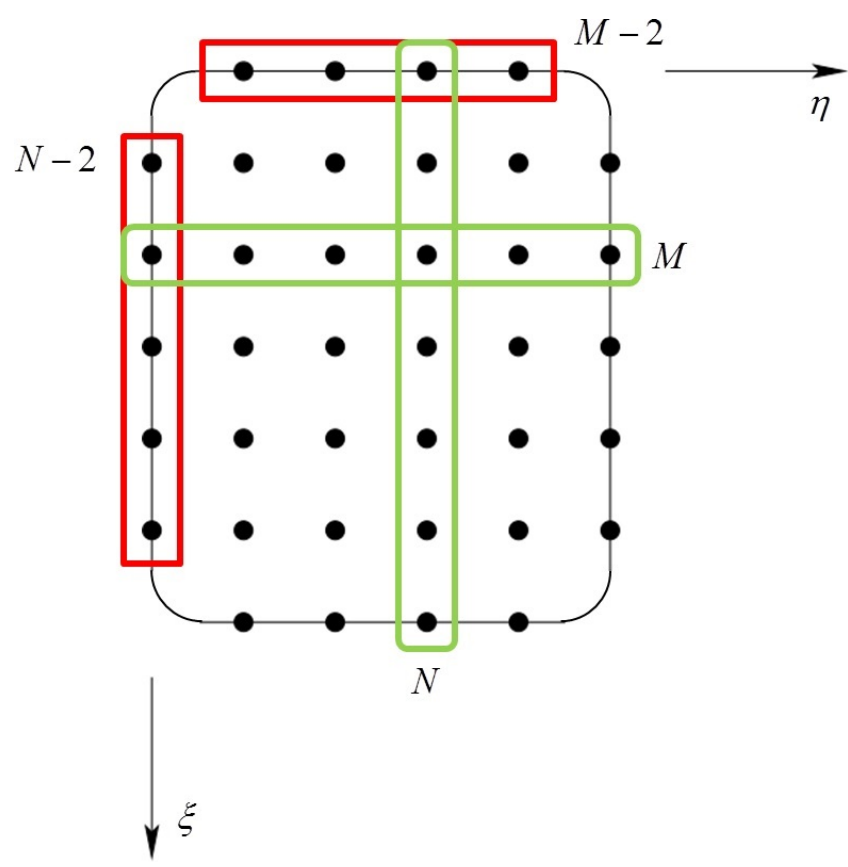

Figure 5: Grid points involved in the derivative approximation, when the corner points are avoided from the implementation. The boxes on the edges are related to the boundary points which have two points less than inner rounded boxes.

$$
\begin{array}{lll}
\mathbf{U}_{n\left(\mathbf{n}_{1}\right)}^{(e)}=0 & & \mathbf{U}_{n\left(\mathbf{n}_{2}\right)}^{(e)}=0 \\
\mathbf{U}_{t\left(\mathbf{n}_{1}\right)}^{(e)}=0 & \text { or } & \mathbf{U}_{t\left(\mathbf{n}_{2}\right)}^{(e)}=0
\end{array}
$$

where $\mathbf{U}_{n}^{(e)}, \mathbf{U}_{t}^{(e)}$ are the algebraic displacement vectors (36) that contain the normal and tangential components to the edge, respectively. The complete symbol $\mathbf{U}_{n\left(\mathbf{n}_{1}\right)}^{(e)}, \mathbf{U}_{t\left(\mathbf{n}_{1}\right)}^{(e)}$

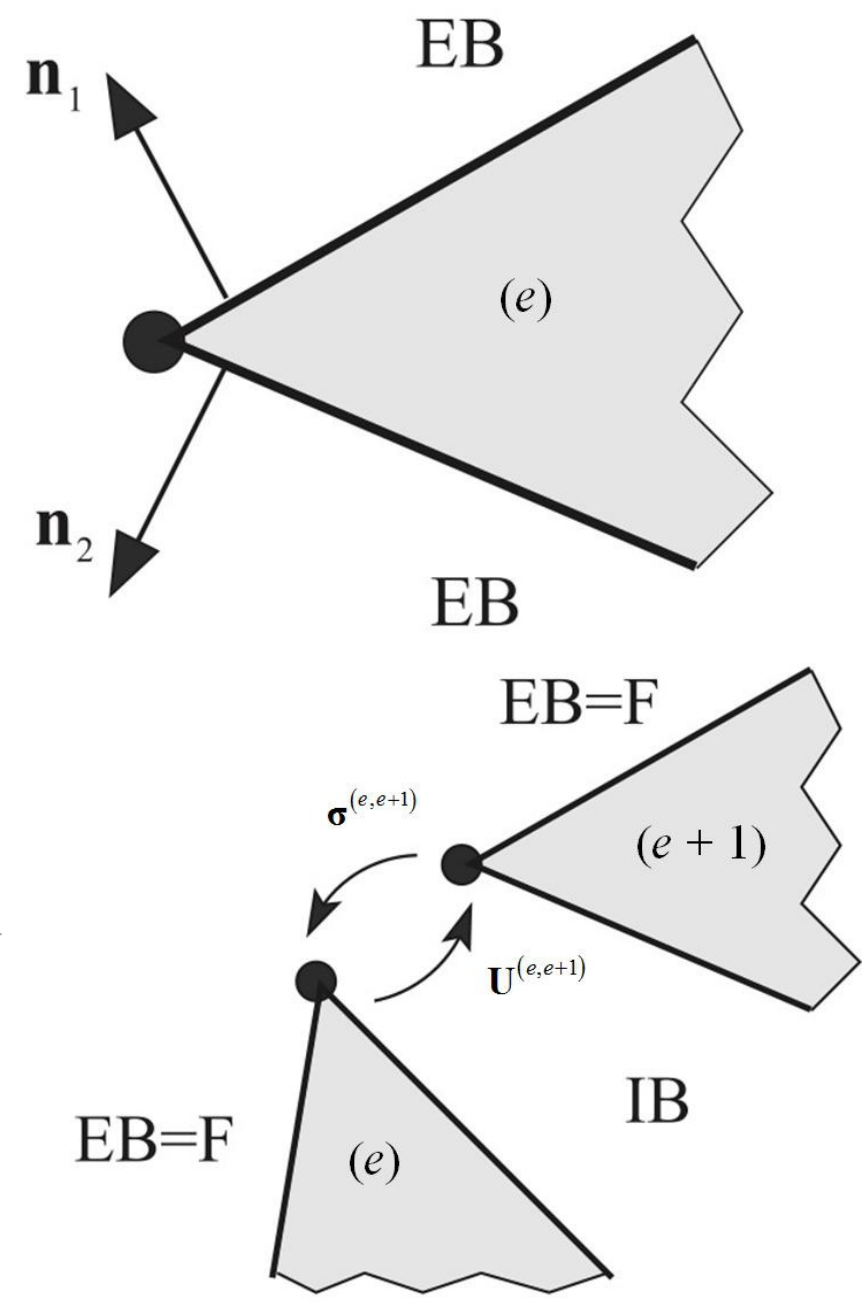

Figure 6: Definition of the external corners conditions for: a) a single element, two facing elements.

means that the algebraic vector contains the normal displacement components of the element ${ }^{(e)}$ with respect to the normal $\mathbf{n}_{1}$. It is important to note that when both edges have a Dirichlet condition the following relation should be set

$$
\mathbf{U}_{n\left(\mathbf{n}_{1}\right)}^{(e)}+\mathbf{U}_{n\left(\mathbf{n}_{2}\right)}^{(e)}=0 \quad \text { and } \quad \mathbf{U}_{t\left(\mathbf{n}_{1}\right)}^{(e)}+\mathbf{U}_{t\left(\mathbf{n}_{2}\right)}^{(e)}=0
$$

It is important to define equation (43) because physically the corner belongs to two edges. Analogously, when both edges are set free (Neumann condition) a similar expression can be reported

$$
\boldsymbol{\sigma}_{n\left(\mathbf{n}_{1}\right)}^{(e)}+\boldsymbol{\sigma}_{n\left(\mathbf{n}_{2}\right)}^{(e)}=0 \quad \text { and } \quad \boldsymbol{\tau}_{n t\left(\mathbf{n}_{1}\right)}^{(e)}+\boldsymbol{\tau}_{n t\left(\mathbf{n}_{2}\right)}^{(e)}=0
$$

where $\boldsymbol{\sigma}_{n}^{(e)}, \boldsymbol{\tau}_{n t}^{(e)}$ are the algebraic stress vectors (37) with the same meaning of the symbols of the previous definitions. Equations (42)- (44) are extremely important when mixed boundary conditions are set, such as the symmetry. It is 
remarked that equations (42)- (44) change when boundary and stress loads are applied to the edge. In particular if a displacement is imposed on the edge with $\mathbf{n}_{1}$, equation (43) becomes

$$
\mathbf{U}_{n\left(\mathbf{n}_{1}\right)}^{(e)}=\overline{\mathbf{U}}_{n} \quad \text { and } \quad \mathbf{U}_{t\left(\mathbf{n}_{1}\right)}^{(e)}=\overline{\mathbf{U}}_{t}
$$

Similarly if a stress load is applied on the edge with $\mathbf{n}_{1}$, equation (44) is

$$
\boldsymbol{\sigma}_{n\left(\mathbf{n}_{1}\right)}^{(e)}=\overline{\boldsymbol{\sigma}}_{n} \quad \text { and } \quad \boldsymbol{\tau}_{n t\left(\mathbf{n}_{1}\right)}^{(e)}=\overline{\boldsymbol{\tau}}_{n t}
$$

Another configuration with two facing elements and an external boundary is depicted in Figure $6 \mathrm{~b}$. The corners of the elements ${ }^{(e)}$ and ${ }^{(e+1)}$ both have an external edge with free conditions (Neumann). The facing edge should be used to set the compatibility conditions (41). Since the continuity condition is physically stronger than the Neumann one, equation (41) are also used in these corners. If boundary loads are enforced the continuity conditions continue to be the best choice for having a better accuracy. A more general configuration is presented in Figure 7 . At the moment a theoretical counterpart of the equations needed for this implementations has not been found yet. For this reason the following numerical trick is proposed, for a general implementation of this kind of configuration. First of all an internal corner is studied, as in Figure $7 \mathrm{a}$, where only internal boundaries (IBs) are present. Second of all an external corner point occur in Figure 7b. It is obvious that for both corners, continuity conditions (41) must be prescribed, with the only exception of one of EB clamped or when boundary loads are set, so equations (42), (45) or (46) must be used. The present approach for multi-corner configuration sets a static (Neumann) condition and several kinematic (Dirichlet) ones. For instance, five elements concur at the displayed node. First, the code identifies the sequence of elements, e.g. 1, 3, 5, 2, 4 and enforce four kinematic conditions (eight algebraic equations), following the first expressions of equation (41) as

$$
\begin{aligned}
& e=1, e+1=3 \rightarrow \mathbf{U}_{n}^{(1)}-\mathbf{U}_{n}^{(3)}=\mathbf{0}, \\
& \mathbf{U}_{t}^{(1)}-\mathbf{U}_{t}^{(3)}=\mathbf{0} \\
& e=3, e+1=5 \rightarrow \mathbf{U}_{n}^{(3)}-\mathbf{U}_{n}^{(5)}=\mathbf{0}, \\
& \mathbf{U}_{t}^{(3)}-\mathbf{U}_{t}^{(5)}=\mathbf{0} \\
& e=5, e+1=2 \rightarrow \mathbf{U}_{n}^{(5)}-\mathbf{U}_{n}^{(2)}=\mathbf{0}, \\
& \mathbf{U}_{t}^{(5)}-\mathbf{U}_{t}^{(2)}=\mathbf{0} \\
& e=2, e+1=4 \rightarrow \mathbf{U}_{n}^{(2)}-\mathbf{U}_{n}^{(4)}=\mathbf{0}, \\
& \mathbf{U}_{t}^{(2)}-\mathbf{U}_{t}^{(4)}=\mathbf{0}
\end{aligned}
$$

Equation (47) can be graphically shortened as

$$
\begin{aligned}
& e=1, e+1=3 \rightarrow \mathbf{U}^{(1,3)} \\
& e=3, e+1=5 \rightarrow \mathbf{U}^{(3,5)} \\
& e=5, e+1=2 \rightarrow \mathbf{U}^{(5,2)} \\
& e=2, e+1=4 \rightarrow \mathbf{U}^{(2,4)}
\end{aligned}
$$

Finally, the static (Neumann) conditions are set between

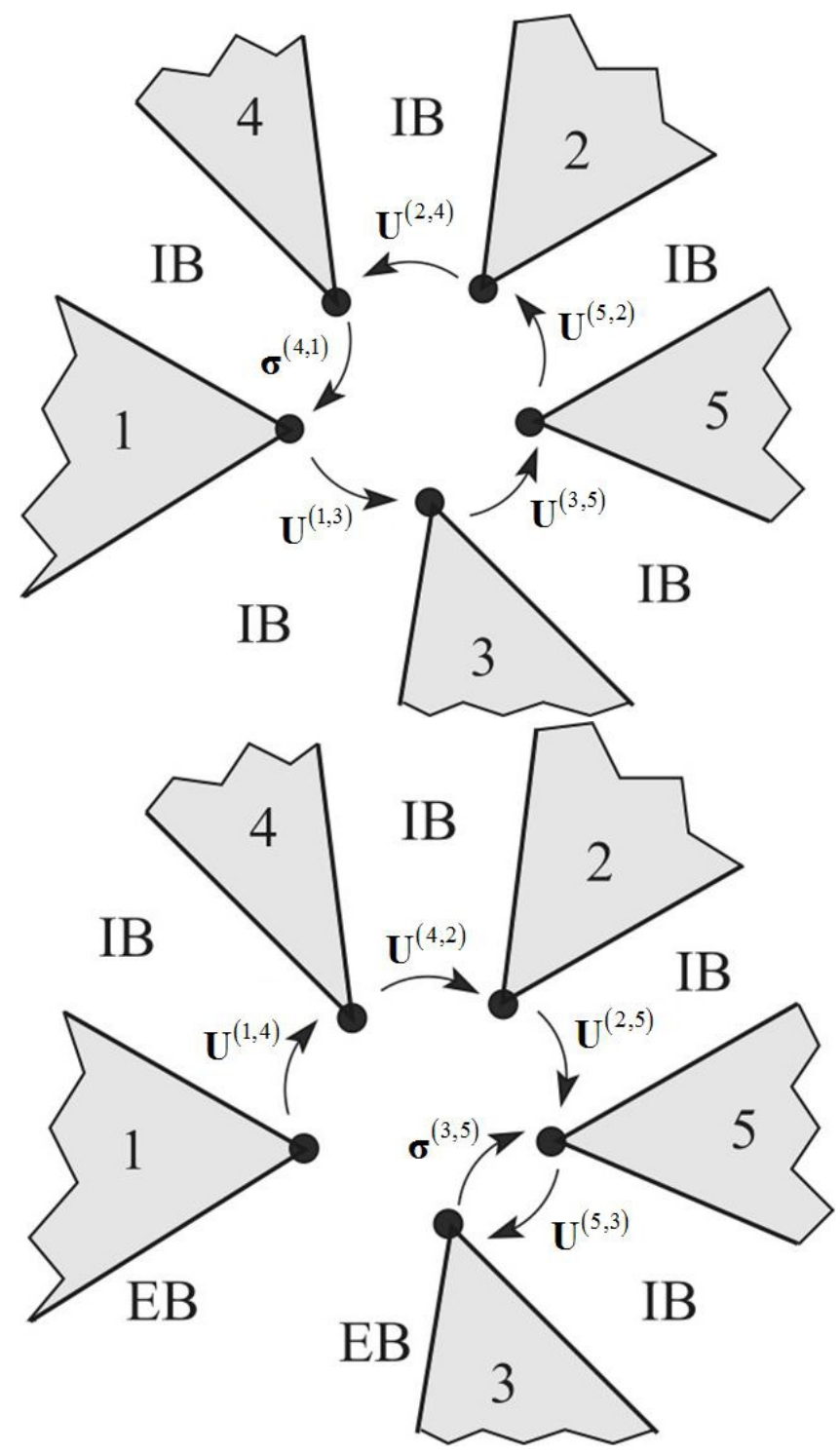

Figure 7: Multiple corner boundary conditions schemes: a) internal corner of five elements with IB conditions; $b$ ) external corner of five elements with $\mathrm{EB}$ and IB conditions.

the last two elements of the group. Following the second expressions of equation (41) they are

$$
e=4, e+1=1 \rightarrow \boldsymbol{\sigma}_{n}^{(4)}-\boldsymbol{\sigma}_{n}^{(1)}=\mathbf{0}, \quad \boldsymbol{\tau}_{n t}^{(4)}-\boldsymbol{\tau}_{n t}^{(1)}=\mathbf{0}
$$

Analogously to the kinematic expressions above, equation (49) can be shortened as

$$
e=4, e+1=1 \rightarrow \sigma^{(4,1)}
$$

In the second configuration of Figure $7 \mathrm{~b}$, considering only external free (Neumann) boundary conditions the following implementation is followed. The previous sequence 
of the element changes, due to the opening, and for the case depicted in Figure $7 \mathrm{~b}$ become 1, 4, 2, 5, 3. With the same meaning of the symbols reported in equations (48) and (50) the following conditions are set

$$
\begin{aligned}
& e=1, e+1=4 \rightarrow \mathbf{U}^{(1,4)} \\
& e=4, e+1=2 \rightarrow \mathbf{U}^{(4,2)} \\
& e=2, e+1=5 \rightarrow \mathbf{U}^{(2,5)} \\
& e=5, e+1=3 \rightarrow \mathbf{U}^{(5,3)} \\
& e=3, e+1=5 \rightarrow \boldsymbol{\sigma}^{(3,5)}
\end{aligned}
$$

It should be pointed out that the conditions $\mathbf{U}^{(e, e+1)}$, $\mathbf{U}^{(e+1, e)}$ or $\boldsymbol{\sigma}^{(e, e+1)}, \boldsymbol{\sigma}^{(e+1, e)}$ are physically the same but numerically different, since they refer to different grid points in the global stiffness matrix. Further details about this aspect will be given in the following subsection. Two other aspects are raised: firstly if the conditions $\mathbf{U}^{(1,3)}, \mathbf{U}^{(3,1)}$ or $\boldsymbol{\sigma}^{(1,3)}, \boldsymbol{\sigma}^{(3,1)}$ were set between the two edges that share external boundaries, inaccurate and unstable numerical solution would occur. Secondly, the corner condition using four static (Neumann) and one kinematic (Dirichlet) equations has been tested, but the accuracy achieved was not sufficient when compared to the present solution. Thus, at the moment, the multi-corner point implementation remains unchanged with respect to the previous published works [126-137].

\subsection{Assemblage}

The final step before the solution is the assembly section. It is a very well-known fact how to assembly a classic FEM algebraic system using $C^{0}$ boundary conditions. On the contrary it is less common to see a $C^{1}$ implementation using strong formulation and continuity conditions. The global structure has the same form of equation (38) where all the sub-matrices are located accordingly. For instance if a mesh is made of three elements the following global system occur

$$
\begin{aligned}
& {\left[\begin{array}{llllll}
\mathbf{K}_{b b}^{(1)} & \mathbf{K}_{b b}^{(1,2)} & \mathbf{K}_{b b}^{(1,3)} & \mathbf{K}_{b d}^{(1)} & \mathbf{K}_{b d}^{(1,2)} & \mathbf{K}_{b d}^{(1,3)} \\
\mathbf{K}_{b b}^{(2,1)} & \mathbf{K}_{b b}^{(2)} & \mathbf{K}_{b b}^{(2,3)} & \mathbf{K}_{b d}^{(2,1)} & \mathbf{K}_{b d}^{(2)} & \mathbf{K}_{b d}^{(2,3)} \\
\mathbf{K}_{b b}^{(3,1)} & \mathbf{K}_{b b}^{(3,2)} & \mathbf{K}_{b b}^{(3)} & \mathbf{K}_{b d}^{(3,1)} & \mathbf{K}_{b d}^{(3,2)} & \mathbf{K}_{b d}^{(3)} \\
\mathbf{K}_{d b}^{(1)} & \mathbf{0} & \mathbf{0} & \mathbf{K}_{d d}^{(1)} & \mathbf{0} & \mathbf{0} \\
\mathbf{0} & \mathbf{K}_{d b}^{(2)} & \mathbf{0} & \mathbf{0} & \mathbf{K}_{d d}^{(2)} & \mathbf{0} \\
\mathbf{0} & \mathbf{0} & \mathbf{K}_{d b}^{(3)} & \mathbf{0} & \mathbf{0} & \mathbf{K}_{d d}^{(3)}
\end{array}\right]} \\
& {\left[\begin{array}{l}
\mathbf{U}_{b}^{(1)} \\
\mathbf{U}_{b}^{(2)} \\
\mathbf{U}_{b}^{(3)} \\
\mathbf{U}_{d}^{(1)} \\
\mathbf{U}_{d}^{(2)} \\
\mathbf{U}_{d}^{(3)}
\end{array}\right]=-\left[\begin{array}{l}
\mathbf{F}_{b}^{(1)} \\
\mathbf{F}_{b}^{(2)} \\
\mathbf{F}_{b}^{(3)} \\
\mathbf{F}_{d}^{(1)} \\
\mathbf{F}_{d}^{(2)} \\
\mathbf{F}_{d}^{(3)}
\end{array}\right]}
\end{aligned}
$$

The first thing that can be noted is that the boundary matrices are full matrices, whereas the domain ones are diagonal as expected, since no connection occurs among the inner points of the elements. Moreover, it should be noted that each sub-matrix contains a particular set of algebraic equations. The matrix form (52) is general and comprehend any configuration. however, some matrices could be empty. For instance, if two elements are not connected $\mathbf{K}_{b b}=\mathbf{0}$ and $\mathbf{K}_{b d}=\mathbf{0}$. moreover, if the kinematic equations between two elements are set, the matrices $\mathbf{K}_{b d}=\mathbf{0}$. In order to give a simpler example, element ${ }^{(1)}$ is connected to element ${ }^{(2)}$ and at the same time element ${ }^{(2)}$ is connected to element ${ }^{(3)}$, but ${ }^{(1)}$ and ${ }^{(3)}$ do not share any boundary. This is the classic case of l-shaped domain. considering these connectivity conditions equation (52) becomes

$$
\begin{aligned}
& {\left[\begin{array}{llllll}
\mathbf{K}_{b b}^{(1)} & \mathbf{K}_{b b}^{(1,2)} & \mathbf{0} & \mathbf{K}_{b d}^{(1)} & \mathbf{0} & \mathbf{0} \\
\mathbf{K}_{b b}^{(2,1)} & \mathbf{K}_{b b}^{(2)} & \mathbf{K}_{b b}^{(2,3)} & \mathbf{K}_{b d}^{(2,1)} & \mathbf{K}_{b d}^{(2)} & \mathbf{0} \\
\mathbf{0} & \mathbf{K}_{b b}^{(3,2)} & \mathbf{K}_{b b}^{(3)} & \mathbf{0} & \mathbf{K}_{b d}^{(3,2)} & \mathbf{K}_{b d}^{(3)} \\
\mathbf{K}_{d b}^{(1)} & \mathbf{0} & \mathbf{0} & \mathbf{K}_{d d}^{(1)} & \mathbf{0} & \mathbf{0} \\
\mathbf{0} & \mathbf{K}_{d b}^{(2)} & \mathbf{0} & \mathbf{0} & \mathbf{K}_{d d}^{(2)} & \mathbf{0} \\
\mathbf{0} & \mathbf{0} & \mathbf{K}_{d b}^{(3)} & \mathbf{0} & \mathbf{0} & \mathbf{K}_{d d}^{(3)}
\end{array}\right]} \\
& {\left[\begin{array}{l}
\mathbf{U}_{b}^{(1)} \\
\mathbf{U}_{b}^{(2)} \\
\mathbf{U}_{b}^{(3)} \\
\mathbf{U}_{d}^{(1)} \\
\mathbf{U}_{d}^{(2)} \\
\mathbf{U}_{d}^{(3)}
\end{array}\right]=-\left[\begin{array}{l}
\mathbf{F}_{b}^{(1)} \\
\mathbf{F}_{b}^{(2)} \\
\mathbf{F}_{b}^{(3)} \\
\mathbf{F}_{d}^{(1)} \\
\mathbf{F}_{d}^{(2)} \\
\mathbf{F}_{d}^{(3)}
\end{array}\right]}
\end{aligned}
$$

It is noted that $\mathbf{K}_{b b}^{(1,3)}=\mathbf{K}_{b d}^{(1,3)}=\mathbf{0}$, since kinematic connectivity is set on the element ${ }^{(1)} \mathbf{K}_{b d}^{(1,2)}=\mathbf{0}$, whereas the static conditions are written on the element ${ }^{(2)}$, thus $\mathbf{K}_{b b}^{(2,1)} \neq \mathbf{0}, \mathbf{K}_{b d}^{(2,1)} \neq \mathbf{0}$. Analogously the connectivity is enforced between elements ${ }^{(2)}$ and ${ }^{(3)}$. In conclusion equation ((52)) can be rewritten in a more compact form as follows

$$
\left[\begin{array}{cc}
\tilde{\mathbf{K}}_{b b} & \tilde{\mathbf{K}}_{b d} \\
\tilde{\mathbf{K}}_{d b} & \tilde{\mathbf{K}}_{d d}
\end{array}\right]\left[\begin{array}{c}
\tilde{\mathbf{U}}_{b} \\
\tilde{\mathbf{U}}_{d}
\end{array}\right]=-\left[\begin{array}{c}
\tilde{\mathbf{F}}_{b} \\
\tilde{\mathbf{F}}_{d}
\end{array}\right]
$$

where $\tilde{\mathbf{K}}_{b b}, \tilde{\mathbf{K}}_{b d}, \tilde{\mathbf{K}}_{d b}, \tilde{\mathbf{K}}_{d d}$ contain the upper-left, upperright, lower-left and lower-right parts of expression (52) respectively. with the similar meaning of the symbols $\tilde{\mathbf{U}}_{b}, \quad \tilde{\mathbf{U}}_{d}, \quad \tilde{\mathbf{F}}_{b}, \quad \tilde{\mathbf{F}}_{d}$ are defined. analogously the global algebraic system for the free vibration problem is represented by the form

$$
\left(\left[\begin{array}{cc}
\tilde{\mathbf{K}}_{b b} & \tilde{\mathbf{K}}_{b d} \\
\tilde{\mathbf{K}}_{d b} & \tilde{\mathbf{K}}_{d d}
\end{array}\right]+\omega^{2}\left[\begin{array}{cc}
\mathbf{0} & \mathbf{0} \\
\mathbf{0} & \tilde{\mathbf{M}}_{d d}
\end{array}\right]\right)\left[\begin{array}{c}
\tilde{\mathbf{U}}_{b} \\
\tilde{\mathbf{U}}_{d}
\end{array}\right]=\left[\begin{array}{c}
\mathbf{0} \\
\mathbf{0}
\end{array}\right]
$$

In order to improve the performance of the final code the static condensation can be carried out for both equa- 
tion (54) and (55). For the static case expression (54) becomes

$$
\begin{aligned}
\tilde{\mathbf{U}}_{b} & =-\tilde{\mathbf{K}}_{b b}^{-1}\left(\tilde{\mathbf{F}}_{b}+\tilde{\mathbf{K}}_{b d} \tilde{\mathbf{U}}_{d}\right) \\
\tilde{\mathbf{U}}_{d} & =\left(\tilde{\mathbf{K}}_{d d}-\tilde{\mathbf{K}}_{d b} \tilde{\mathbf{K}}_{b b}^{-1} \tilde{\mathbf{K}}_{b d}\right)^{-1}\left(\tilde{\mathbf{K}}_{d b} \tilde{\mathbf{K}}_{b b}^{-1} \tilde{\mathbf{F}}_{b}-\tilde{\mathbf{F}}_{d}\right)
\end{aligned}
$$

And the dynamic case (55) can be rewritten as

$$
\begin{aligned}
& \tilde{\mathbf{U}}_{b}=-\tilde{\mathbf{K}}_{b b}^{-1} \tilde{\mathbf{K}}_{b d} \tilde{\mathbf{U}}_{d} \\
& \left(\left(\tilde{\mathbf{K}}_{d d}-\tilde{\mathbf{K}}_{d b} \tilde{\mathbf{K}}_{b b}^{-1} \tilde{\mathbf{K}}_{b d}\right)+\omega^{2} \tilde{\mathbf{M}}_{d d}\right) \tilde{\mathbf{U}}_{d}=\mathbf{0}
\end{aligned}
$$

For the first case, the second equation of expression (56) is solved by gaussian elimination and the boundary displacements are retrieved using the first equation of (56). In the latter study, the generalized eigenvalue problem is solved (the second expression of (57)). Once the mode shapes of the inner points $\tilde{\mathbf{U}}_{d}$ are evaluated, the boundary quantities are retrieved using the first equation in (57).

\section{Validation studies}

As most of the numerical methodologies based on domain decomposition or finite elements, the present numerical approach suffers from two main issues: one is due to the accuracy inside each element (related to the derivative approximation), the other one is brought by the mapping technique and the element distortion. For these reasons several validation tests are presented in order to show and demonstrate what has been hitherto cited. It is not the purpose of the present work to investigate the behavior of the technique using several basis functions and point collocations. Thus, the most well-known accurate method has been considered: Lagrange polynomials (known also as Polynomial Differential Quadrature (PDQ)) and Chebyshev-Gauss-Lobatto (C-G-L) grid. For further details about this the reader can refer to the review papers $[136,137]$. It is recalled that PDQ has been introduced by Shu [20] and the C-G-L grid has a non-uniform distribution which takes the following form in the master element

$$
\begin{aligned}
& \xi_{i}=\cos \left(\frac{N-i}{N-1} \pi\right), \quad i=1,2, \ldots, N \\
& \eta_{j}=\cos \left(\frac{M-j}{M-1} \pi\right), \quad j=1,2, \ldots, M
\end{aligned}
$$

All the following computations have been carried out changing the number of grid points $N, M$ inside each element and the number of elements $n_{e}$ used for the mesh subdivision. In particular, emphasis has been put on the use of several boundary conditions, especially the results related to the use of the mixed ones (such as the symmetry), since this has never been applied before [126-137].

\subsection{Free in-plane vibrations of a square plate}

In order to first present the good accuracy of the present methodology the well-known case of the in-plane free vibrations of a square plate is given in the following. Several articles presented this problem in literature in previous published papers [139-141]. Thus, the past solutions are used here as a benchmark for the present code. Furthermore, some observations can be made while modeling this structure. It is a very well-known fact that the easiest boundary condition for the GDQ method is the kinematic (fixed, Dirichlet) one. This is self-explained by the fact that the problem is solved using the partial differential system of equations as a function of the displacements. Thus, using the identity matrix as matrix for the boundary conditions, automatically all the edges are fixed. On the contrary, it is more difficult to enforce the natural (free, Neumann) boundary conditions, since the conditions on the stresses also comprehend the derivatives of the displacements. For this reason the first comparison is given with respect to a fully clamped (C-C-C-C) plate using a single element and a mesh made of four elements. The plate subdivision is performed using regular elements, so that the element dimensions are given by the plate edge divided by the number of element per edge. In other words if $a$ and $b$ identify the two edges of the plate, when four elements are considered each element has dimension $a / 2$ and $b / 2$. It is recalled that using a regular subdivision (a multi-domain technique is under consideration) a different number of points per side can be considered. This is particularly important when a rectangular plate is taken into account $(a / b=2)$, whereas for a square plate $(a / b=1)$ it is better to have $N=M$. A FEM model is also presented using Abaqus, with a regular $(100 \times 100$ for $a / b=1$ and $200 \times 100$ for $a / b=2$ ) mesh made of CPS8 elements. The results related to a C-C-C-C plate are shown in Table 1. The natural frequencies are presented in their dimensionless form as $\Omega=\omega a \sqrt{\rho\left(1-v^{2}\right) / E}$, where $\omega$ indicates the circular frequency. The case of a completely free (F-F-F-F) plate is reported in Table 2, where only Neumann conditions are used. For both cases the same number of grid points is used and it is noted that when $N \neq M$ in the rectangular plate model, the results are more accurate than the case with $N=M$. This effect is more noticeable when $a \gg b$ like 5 or 10 times. It is also remarked that the present solutions agree with the results presented by other authors in literature. 
Table 1: First ten dimensionless frequencies for C-C-C-C isotropic in-plane square and rectangular plates using different techniques.

\begin{tabular}{|c|c|c|c|c|c|c|c|}
\hline \multicolumn{8}{|c|}{$a / b=1$} \\
\hline$\Omega$ & Ref. [139] & Ref. [140] & $\begin{array}{c}n_{e}=1 \\
N=M=7\end{array}$ & $\begin{array}{c}n_{e}=1 \\
N=M=21\end{array}$ & $\begin{array}{c}n_{e}=4 \\
N=M=7\end{array}$ & $\begin{array}{c}n_{e}=4 \\
N=M=11\end{array}$ & $\begin{array}{c}\text { FEM } \\
(\text { CPS } 8) \\
100 \times 100\end{array}$ \\
\hline 1 & 3.555 & 3.549 & 3.55507 & 3.55519 & 3.55521 & 3.55518 & 3.55519 \\
\hline 2 & 3.555 & 3.549 & 3.55507 & 3.55519 & 3.55521 & 3.55518 & 3.55519 \\
\hline 3 & 4.235 & 4.221 & 4.23521 & 4.23501 & 4.23587 & 4.23501 & 4.23501 \\
\hline 4 & 5.186 & 5.201 & 5.19662 & 5.18570 & 5.18481 & 5.18570 & 5.18571 \\
\hline 5 & 5.859 & 5.967 & 5.83147 & 5.85862 & 5.85948 & 5.85862 & 5.85861 \\
\hline 6 & 5.895 & 6.000 & 5.83147 & 5.89441 & 5.89867 & 5.89442 & 5.89442 \\
\hline 7 & - & - & 5.86942 & 5.89441 & 5.89867 & 5.89442 & 5.89442 \\
\hline 8 & - & - & 6.72321 & 6.70768 & 6.70674 & 6.70767 & 6.70768 \\
\hline 9 & - & - & 7.10014 & 7.11317 & 7.11554 & 7.11317 & 7.11318 \\
\hline 10 & - & - & 7.10014 & 7.11317 & 7.11554 & 7.11317 & 7.11318 \\
\hline \multicolumn{8}{|c|}{$a / b=2$} \\
\hline$\Omega$ & Ref. [139] & Ref. [140] & $\begin{array}{c}n_{e}=1 \\
N=M=7\end{array}$ & $\begin{array}{c}n_{e}=1 \\
N=21, M=11\end{array}$ & $\begin{array}{c}n_{e}=4 \\
N=11, M=7\end{array}$ & $\begin{array}{c}n_{e}=4 \\
N=21, M=11\end{array}$ & $\begin{array}{c}\text { FEM } \\
(\text { CPS } 8) \\
200 \times 100\end{array}$ \\
\hline 1 & 4.789 & 4.741 & 4.78860 & 4.78902 & 4.78903 & 4.78902 & 4.78903 \\
\hline 2 & 6.379 & 6.387 & 6.37838 & 6.37856 & 6.37860 & 6.37856 & 6.37855 \\
\hline 3 & 6.712 & 6.682 & 6.71533 & 6.71212 & 6.71238 & 6.71213 & 6.71213 \\
\hline 4 & 7.049 & 7.037 & 7.05019 & 7.04875 & 7.04885 & 7.04875 & 7.04878 \\
\hline 5 & 7.608 & 7.565 & 7.61663 & 7.60830 & 7.60877 & 7.60831 & 7.60827 \\
\hline 6 & 8.140 & 8.128 & 8.20558 & 8.14019 & 8.14008 & 8.14019 & 8.14022 \\
\hline 7 & - & - & 9.33430 & 8.99796 & 8.99838 & 8.99797 & 8.99795 \\
\hline 8 & - & - & 9.78770 & 9.51559 & 9.51617 & 9.51560 & 9.51557 \\
\hline 9 & - & - & 10.17764 & 9.71655 & 9.71624 & 9.71655 & 9.71655 \\
\hline 10 & - & - & 10.94940 & 10.60077 & 10.60080 & 10.60079 & 10.60078 \\
\hline
\end{tabular}

\subsection{Free in-plane vibrations of a circular plate}

In the present subsection, the mapping technique is introduced and tested. The reference article is the one by Park [142], where a clamped isotropic circular plate is investigated. The plate has a radius of $0.5 \mathrm{~m}$ and it is made of Aluminium with Young's modulus of $71 \mathrm{GPa}$, Poisson's ratio of 0.33 and density $2700 \mathrm{~kg} / \mathrm{m}^{3}$. The problem of annular and circular plates is very well-known in literature [143]. The results are proposed for two different meshes with $n_{e}=4$ and $n_{e}=12$. The elements have 8 nodes in order to map correctly the curvature. The meshes used are the same as the ones used in the previous works [126, 133, 134]. Table 3 shows the comparisons with the results proposed by Park and also a FEM solution using 9802 CPS8 elements. Good agreement is observed for the first 25 natural frequencies. It is underlined that the reference articles show only the symmetric frequencies, whereas the present solution and the FEM report all the physical quantities of the structure.

\subsection{Static analysis of a thick walled cylinder}

Consider the standard thick-walled cylinder test presented by MacNeal and Harder [144]. In this example, the structure is under plane strain conditions and simulate a thickwalled cylinder (of infinite length) subjected to an internal pressure $p$. A 10 degrees segment is modeled with an inner radius $\mathrm{R}_{i}=3 \mathrm{~m}$ and an outer radius $\mathrm{R}_{o}=9 \mathrm{~m}$. In the present analysis the radial, tangential and longitudinal stresses are evaluated and compared to an exact solution given in literature. Furthermore, the radial displacement is also presented. The analyses are carried out considering different number of Poisson's ratios $v=$ 
Table 2: First ten dimensionless frequencies for F-F-F-F isotropic in-plane square and rectangular plates using different techniques.

\begin{tabular}{|c|c|c|c|c|c|c|c|}
\hline \multicolumn{8}{|c|}{$a / b=1$} \\
\hline$\Omega$ & Ref. [139] & Ref. [140] & $\begin{array}{c}n_{e}=1 \\
N=M=7\end{array}$ & $\begin{array}{c}n_{e}=1 \\
N=M=21\end{array}$ & $\begin{array}{c}n_{e}=4 \\
N=M=7\end{array}$ & $\begin{array}{c}n_{e}=4 \\
N=M=11\end{array}$ & $\begin{array}{c}\text { FEM } \\
(\text { CPS8) } \\
100 \times 100\end{array}$ \\
\hline 1 & 2.321 & 2.321 & 2.32451 & 2.32171 & 2.31605 & 2.32145 & 2.32060 \\
\hline 2 & 2.472 & 2.472 & 2.45872 & 2.46801 & 2.45043 & 2.47238 & 2.47162 \\
\hline 3 & 2.472 & 2.472 & 2.48889 & 2.46801 & 2.45388 & 2.47641 & 2.47162 \\
\hline 4 & 2.628 & 2.628 & 2.62683 & 2.62859 & 2.62428 & 2.62825 & 2.62845 \\
\hline 5 & 2.987 & 2.987 & 2.96949 & 2.98631 & 2.97801 & 2.98617 & 2.98738 \\
\hline 6 & 3.452 & 3.452 & 3.45888 & 3.44945 & 3.45045 & 3.44062 & 3.45224 \\
\hline 7 & - & - & 3.69459 & 3.71042 & 3.68715 & 3.72062 & 3.72313 \\
\hline 8 & - & - & 3.69459 & 3.71244 & 3.68902 & 3.72062 & 3.72313 \\
\hline 9 & - & - & 4.21216 & 4.31437 & 4.18625 & 4.32203 & 4.30307 \\
\hline 10 & - & - & 4.72090 & 4.96327 & 4.80540 & 4.96643 & 4.96863 \\
\hline \multicolumn{8}{|c|}{$a / b=2$} \\
\hline$\Omega$ & Ref. [139] & Ref. [140] & $\begin{array}{c}n_{e}=1 \\
N=M=7\end{array}$ & $\begin{array}{c}n_{e}=1 \\
N=21, M=11\end{array}$ & $\begin{array}{c}n_{e}=4 \\
N=11, M=7\end{array}$ & $\begin{array}{c}n_{e}=4 \\
N=21, M=11\end{array}$ & $\begin{array}{c}\text { FEM } \\
(\text { CPS } 8) \\
200 \times 100 \\
\end{array}$ \\
\hline 1 & 1.954 & 1.938 & 1.96633 & 1.95803 & 1.95933 & 1.94801 & 1.95365 \\
\hline 2 & 2.961 & 2.927 & 2.96229 & 2.95793 & 2.95905 & 2.96102 & 2.96082 \\
\hline 3 & 3.267 & 3.238 & 3.30916 & 3.29986 & 3.26824 & 3.26463 & 3.26705 \\
\hline 4 & 4.726 & 4.702 & 4.61846 & 4.68942 & 4.72633 & 4.72400 & 4.72633 \\
\hline 5 & 4.784 & 4.752 & 4.68062 & 4.76869 & 4.79928 & 4.81284 & 4.78411 \\
\hline 6 & 5.205 & 5.178 & 5.24640 & 5.21348 & 5.21344 & 5.19110 & 5.20445 \\
\hline 7 & - & - & 5.30536 & 5.27862 & 5.25165 & 5.26765 & 5.25689 \\
\hline 8 & - & - & 5.64674 & 5.39243 & 5.37669 & 5.35378 & 5.36510 \\
\hline 9 & - & - & 6.02023 & 6.15741 & 6.09865 & 6.15654 & 6.14655 \\
\hline 10 & - & - & 6.08108 & 6.41060 & 6.43985 & 6.46616 & 6.44752 \\
\hline
\end{tabular}

$(0.3,0.49,0.499,0.4999)$ and the elastic modulus is set equal to $\mathrm{E}=1 \mathrm{~Pa}$. The plane strain exact solution can be found in the book by Timoshenko [2]. The equation for the radial displacement is

$$
\frac{d}{d r}\left(\frac{1}{r} \frac{d}{d r}\left(r u_{r}\right)\right)=0
$$

where $u_{r}$ indicates the radial displacement and $r$ is the radial coordinate. Integrating two times equation (59), the following expression for the radial displacement comes out

$$
u_{r}=a r+\frac{b}{r}
$$

where $a, b$ are two integration constants that can be easily derived as

$$
a=(1-2 v) \frac{b}{R_{o}^{2}} \quad b=\frac{-p(1+v)}{E\left(\frac{1}{R_{o}^{2}}-\frac{1}{r^{2}}\right)}
$$

Once the radial displacement is defined the stress quantities can be evaluated afterwards as follows

$$
\begin{aligned}
& \sigma_{r}=\frac{E}{1+v}\left(\frac{d u_{r}}{d r}+\frac{v}{1-2 v}\left(\frac{d u_{r}}{d r}+\frac{u_{r}}{r}\right)\right) \\
& \sigma_{t}=\frac{E}{1+v}\left(\frac{u_{r}}{r}+\frac{v}{1-2 v}\left(\frac{d u_{r}}{d r}+\frac{u_{r}}{r}\right)\right) \\
& \sigma_{z}=\frac{E}{1+v}\left(\frac{v}{1-2 v}\left(\frac{d u_{r}}{d r}+\frac{u_{r}}{r}\right)\right)
\end{aligned}
$$

It is remarked form equation (62) that $\sigma_{z} \neq 0$ since a plane strain conditions has been considered. The results of the present case are reported in Table 4 for three different meshes that are depicted in Figure 8 using different number of grid points. In each case the number of degrees of freedom is kept relatively high, using polynomials of high degree in order to catch the solution with a small number of finite elements and a small error. Table 4 reports not only the numerical solution but also the percentage of the 
Table 3: First twenty-five frequencies for C-C-C-C isotropic circular plate using different techniques.

\begin{tabular}{|c|c|c|c|c|c|}
\hline$f[H z]$ & Ref. [142] & Ref. [142] & FEM (CPS8) $n_{e}=9802$ & $n_{e}=12 N=M=7$ & $n_{e}=4 N=M=15$ \\
\hline 1 & 3363.6 & 3362 & 3361.73 & 3363.074 & 3363.044 \\
\hline 2 & - & - & 3361.73 & 3363.074 & 3363.044 \\
\hline 3 & 3836.4 & 3835 & 3834.85 & 3836.446 & 3836.351 \\
\hline 4 & 5217.5 & 5219 & 5219.37 & 5221.504 & 5221.404 \\
\hline 5 & - & - & 5219.37 & 5221.522 & 5221.407 \\
\hline 6 & 5380.5 & 5383 & 5382.86 & 5385.225 & 5384.962 \\
\hline 7 & - & - & 5382.86 & 5385.225 & 5384.962 \\
\hline 8 & 6624 & 6626 & 6625.62 & 6628.278 & 6628.200 \\
\hline 9 & 6749.3 & 6764 & 6763.75 & 6767.236 & 6766.389 \\
\hline 10 & - & - & 6763.75 & 6767.236 & 6766.389 \\
\hline 11 & 6929 & 6939 & 6938.50 & 6941.662 & 6941.210 \\
\hline 12 & - & - & 6938.50 & 6941.838 & 6941.214 \\
\hline 13 & 7019.3 & 7021 & 7021.35 & 7024.409 & 7024.091 \\
\hline 14 & 8093 & 8130 & 8130.48 & 8134.337 & 8133.058 \\
\hline 15 & - & - & 8130.48 & 8137.422 & 8134.240 \\
\hline 16 & 8476.5 & 8489 & 8489.44 & 8496.113 & 8492.753 \\
\hline 17 & - & - & 8489.44 & 8496.113 & 8492.753 \\
\hline 18 & 8530.6 & 8557 & 8557.33 & 8562.647 & 8560.662 \\
\hline 19 & - & - & 8557.33 & 8562.647 & 8560.662 \\
\hline 20 & 9258 & 9263 & 9262.99 & 9266.436 & 9266.602 \\
\hline 21 & - & - & 9262.99 & 9266.436 & 9266.602 \\
\hline 22 & 9328.1 & 9401 & 9401.23 & 9409.051 & 9404.896 \\
\hline 23 & - & - & 9401.23 & 9409.051 & 9404.896 \\
\hline 24 & 9887.7 & 9925 & 9925.01 & 9932.331 & 9928.872 \\
\hline 25 & - & - & 9925.01 & 9935.469 & 9928.876 \\
\hline
\end{tabular}

relative error. The numerical solutions are in good agreement with the exact solution provided by hand calculations, for different values of Poisson's ratio and number of elements. In fact, the error computed is very small in all cases. In order to complete the analysis a convergence test is carried out in Figure 9 where the number of degrees of freedom (dofs) is increasing keeping the same number of elements. The three curves obtained using SFEM are compared to a FEM solution obtained in Straus. The exact solution is used as a reference for computing the error along the vertical axis of the plot. From the double-log plot it can be noticed as expected that the convergence ratio of the SFEM is steeper than the FEM due to the high-order polynomial approximation.

\subsection{Sensitivity analysis of a composite thick walled cylinder}

Simulating the previous example a composite case is deducted. In particular, the aim of this application is to investigate the sensitivity of the present geometry when two different materials are considered. The mesh used in the computations is depicted in Figure 10. The geometry is kept the same as the previous case, whereas the value of the elastic modulus of the inner and outer sheets is variable using $10,100,1000$. All the quantities are evaluated at the inner radius as it can be deducted from the radial stress that is equal to the external applied load. The results presented in Table 5 are aimed to be used as a reference for further studies on the subject. 


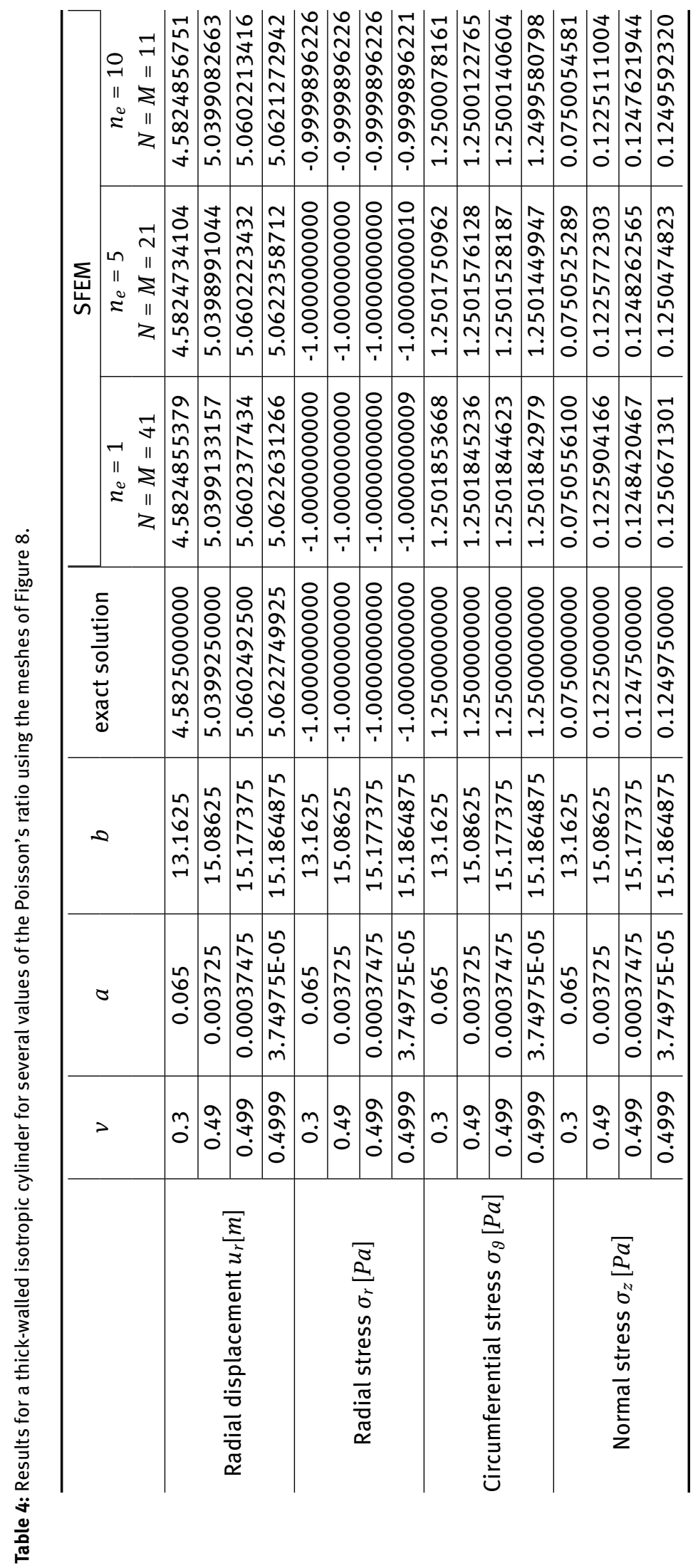


Table 5: Results for a thick-walled composite cyilinder for several values of the Poisson's ratio and the ratio between the core and sheet moduli using $21 \times 21$ grid points per element and the meshes of Figure 10 .

\begin{tabular}{|c|c|c|c|c|c|c|c|}
\hline & \multirow{2}{*}{$v$} & \multicolumn{2}{|l|}{$E / E_{c}=10$} & \multicolumn{2}{|c|}{$E / E_{c}=100$} & \multicolumn{2}{|c|}{$E / E_{c}=1000$} \\
\hline & & $n_{e}=3$ & $n_{e}=5$ & $n_{e}=3$ & $n_{e}=5$ & $n_{e}=3$ & $n_{e}=5$ \\
\hline \multirow{4}{*}{ Radial displacement $u_{r}[\mathrm{~m}]$} & 0.3 & 12.14834 & 12.14834 & 15.48735 & 15.48735 & 16.21028 & 16.21028 \\
\hline & 0.49 & 11.91896 & 11.91895 & 13.92165 & 13.92165 & 14.53872 & 14.53872 \\
\hline & 0.499 & 11.88706 & 11.88705 & 13.75455 & 13.75455 & 14.08313 & 14.08313 \\
\hline & 0.4999 & 11.88384 & 11.88381 & 13.73592 & 13.73592 & 13.96643 & 13.96643 \\
\hline \multirow{4}{*}{ Radial stress $\sigma_{r}[\mathrm{~Pa}]$} & 0.3 & -0.99999 & -0.99999 & -0.99999 & -0.99999 & -0.99999 & -0.99999 \\
\hline & 0.49 & -0.99999 & -0.99999 & -0.99999 & -0.99999 & -0.99999 & -0.99999 \\
\hline & 0.499 & -0.99999 & -0.99999 & -0.99999 & -0.99999 & -0.99999 & -0.99999 \\
\hline & 0.4999 & -0.99999 & -0.99999 & -0.99999 & -0.99999 & -0.99999 & -0.99999 \\
\hline \multirow{4}{*}{ Circumferential stress $\sigma_{\vartheta}[\mathrm{Pa}]$} & 0.3 & 4.02352 & 4.02352 & 5.24712 & 5.24712 & 5.51205 & 5.51205 \\
\hline & 0.49 & 4.26979 & 4.26979 & 5.14866 & 5.14866 & 5.41946 & 5.41946 \\
\hline & 0.499 & 4.28238 & 4.28238 & 5.11164 & 5.11164 & 5.25754 & 5.25754 \\
\hline & 0.4999 & 4.28367 & 4.28365 & 5.10707 & 5.10707 & 5.20955 & 5.20955 \\
\hline \multirow{4}{*}{ Normal stress $\sigma_{z}[\mathrm{~Pa}]$} & 0.3 & 0.90706 & 0.90706 & 1.27414 & 1.27414 & 1.35362 & 1.35362 \\
\hline & 0.49 & 1.60220 & 1.60220 & 2.03285 & 2.03285 & 2.16554 & 2.16554 \\
\hline & 0.499 & 1.63791 & 1.63791 & 2.05171 & 2.05171 & 2.12452 & 2.12452 \\
\hline & 0.4999 & 1.64151 & 1.64150 & 2.05313 & 2.05313 & 2.10436 & 2.10436 \\
\hline
\end{tabular}

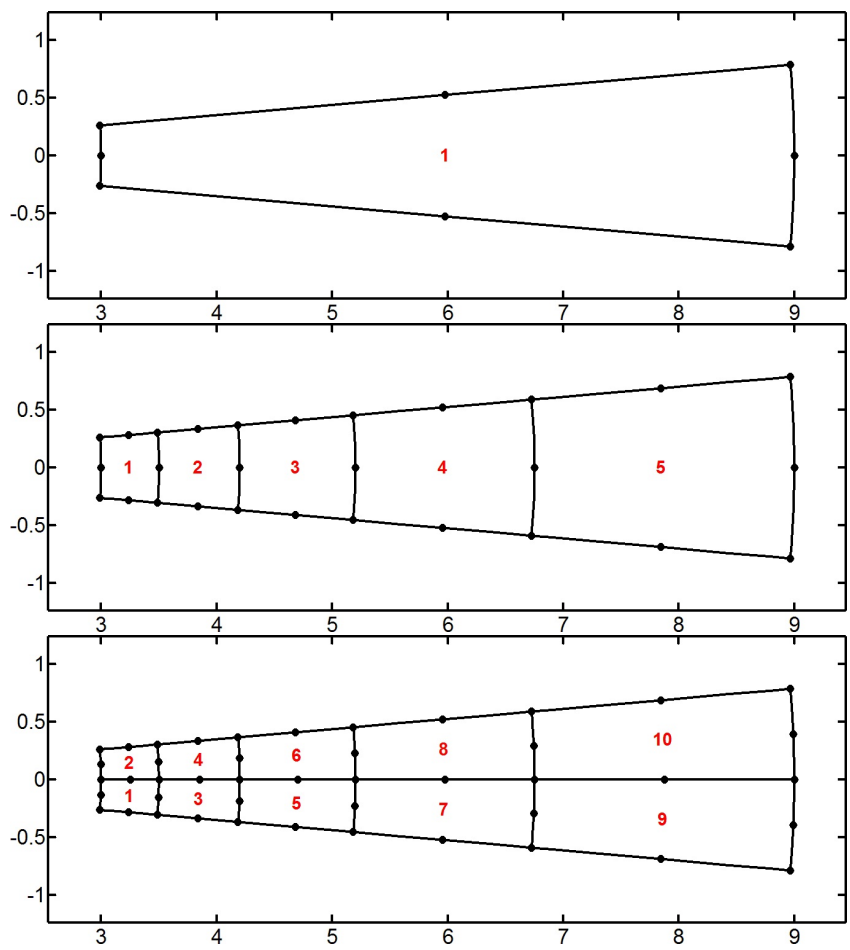

Figure 8: Isotropic and composite thick-walled cylinder meshes used for the convergence computations.

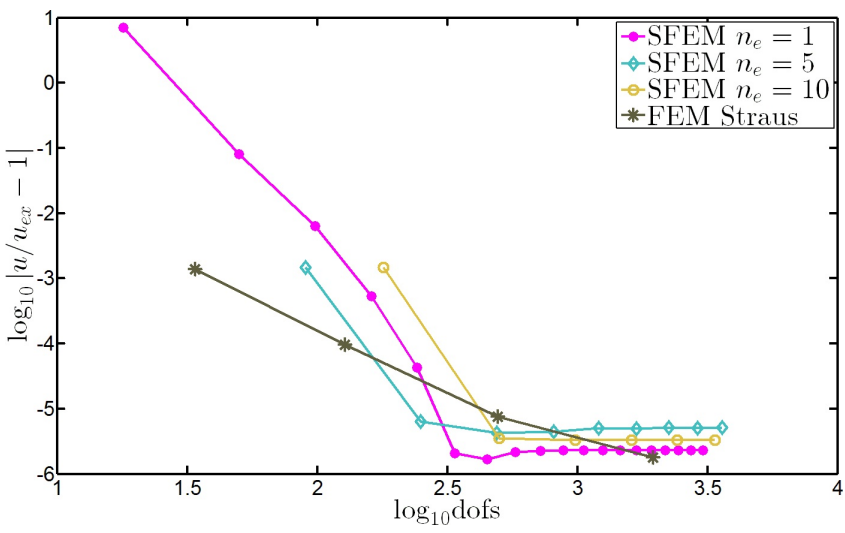

Figure 9: Convergence and stability curves for an isotropic thickwalled cylinder using different meshes.

\subsection{D elastic structure made of two different materials}

The present numerical application has been taken from the book by Zong and Zhang [122], where a 2D rectangular $12 \mathrm{~m} \times 6 \mathrm{~m}$ body has a lateral traction $\mathrm{q}=10 \mathrm{~Pa}$. The structure can be studied using a doubly symmetry on the $x$ and $y$ axis and the whole domain can be just divided into two squared elements $n_{e}=2$. In this way a composite structure can be studied without considering the mapping technique. The mechanical properties of the half on the left are $E_{1}=3 \cdot 10^{7} \mathrm{~Pa}, v_{1}=0.25$, whereas the ones of the half on 

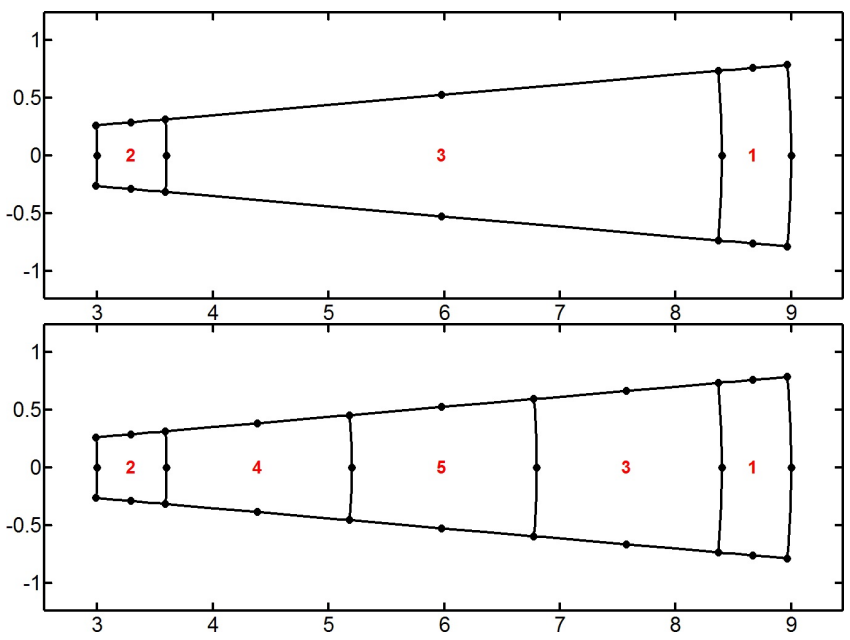

Figure 10: Composite thick-walled cylinder meshes used for the convergence computations.

the right are $E_{2}=3 \cdot 10^{6} \mathrm{~Pa}, v_{1}=0.25$. The results are compared in terms of stresses $\sigma_{x}$ and $\tau_{x y}$, at section $y=1.5 \mathrm{~m}$. The SFEM solution is superimposed to the reference ones using $N=M=21$ in Figure 11. The black solid line is the solution proposed by Zong and Zhang and the black circles are related to a FEM (Abaqus) solution obtained by Zong and Zhang. Extremely good agreement is observed and this example shows that it is possible to investigate composite structures easily with the present method.
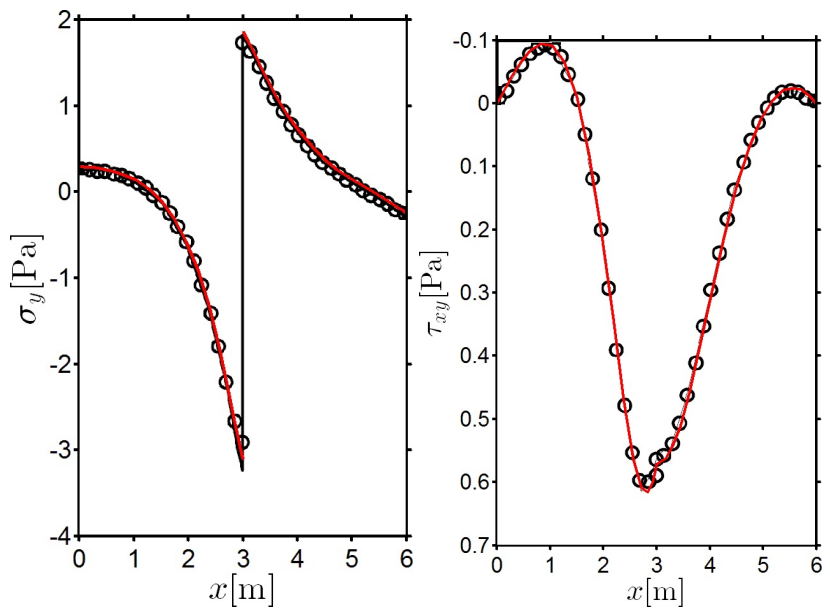

Figure 11: Stress distributions at $y=H / 2$ for a bi-material beam. The present result using $N=M=21$ for each element is compared to the same presented by Zong and Zhang [122], who solved the problem using multi-domain DQ and FEM (Abaqus).

\subsection{Square plate with a square inclusion}

Another application taken from the book by Zong and Zhang [122] considers a square plate with a square inclusion subjected to a horizontal traction $\mathrm{q}=100 \mathrm{~Pa}$. The problem has a double symmetry so a quarter of the plate can be studied. For the present case four regular (squared) elements are used $n_{e}=4$. The material properties for the inclusion are $E_{1}=3 \cdot 10^{6} \mathrm{~Pa}, v_{1}=0.25$ and the ones for the matrix are $E_{1}=3 \cdot 10^{7} \mathrm{~Pa}, v_{1}=0.3$. The dimension of the quarter of the plate is $L=2 \mathrm{~m}$ and the side of the squared inclusion is $L / 2=1 \mathrm{~m}$. The results are presented in terms of displacements $u=u_{x}$ and $v=u_{y}$ in Figure 12, where the present solution with a solid line is compared to the solutions proposed by Zong and Zhang [122]. The black solid line is the Abaqus solution, the stars and triangles markers are the solutions proposed by Zong and Zhang [122]. Figure 12 is obtained drawing a section at $y=L / 4=0.5 \mathrm{~m}$. Analogously at the same section the stresses $\sigma_{x}, \sigma_{y}$ and $\tau_{x y}$ are shown in Figure 13. The present solution is obtained using $N=M=21$. A good agreement is observed, even though some differences can be seen at the material discontinuity interface, since a difference between the present solution and the others occurs. Thus, Figure 14 is presented where another FEM solution has been carried out using a very fine mesh and it can be noted that the present results are in very good agreement with this new FEM reference solution. This is due to the fact that Zong and Zhang used a coarse Abaqus mesh for their calculations.
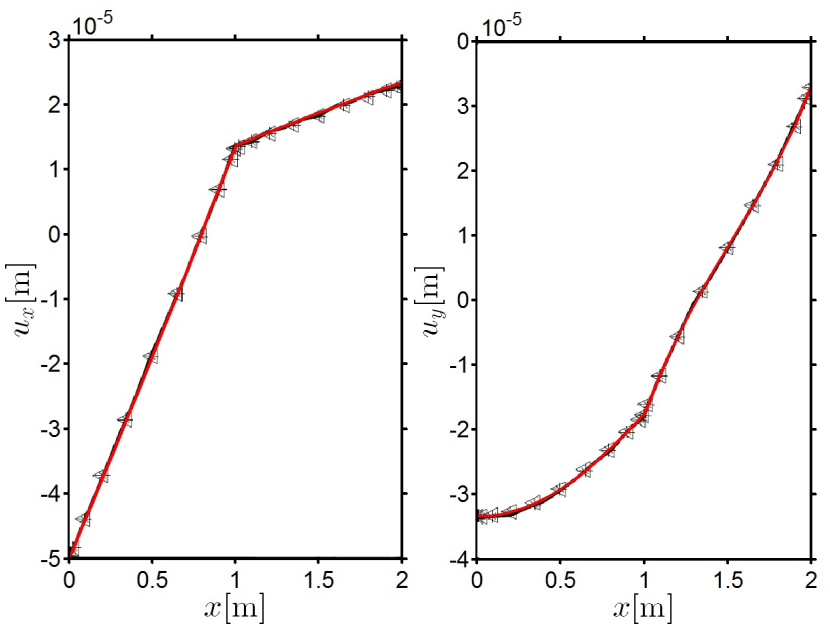

Figure 12: The present result using $N=M=21$ for each element is compared to the same presented by Zong and Zhang [122], who solved the problem using multi-domain DQ and FEM (Abaqus). 

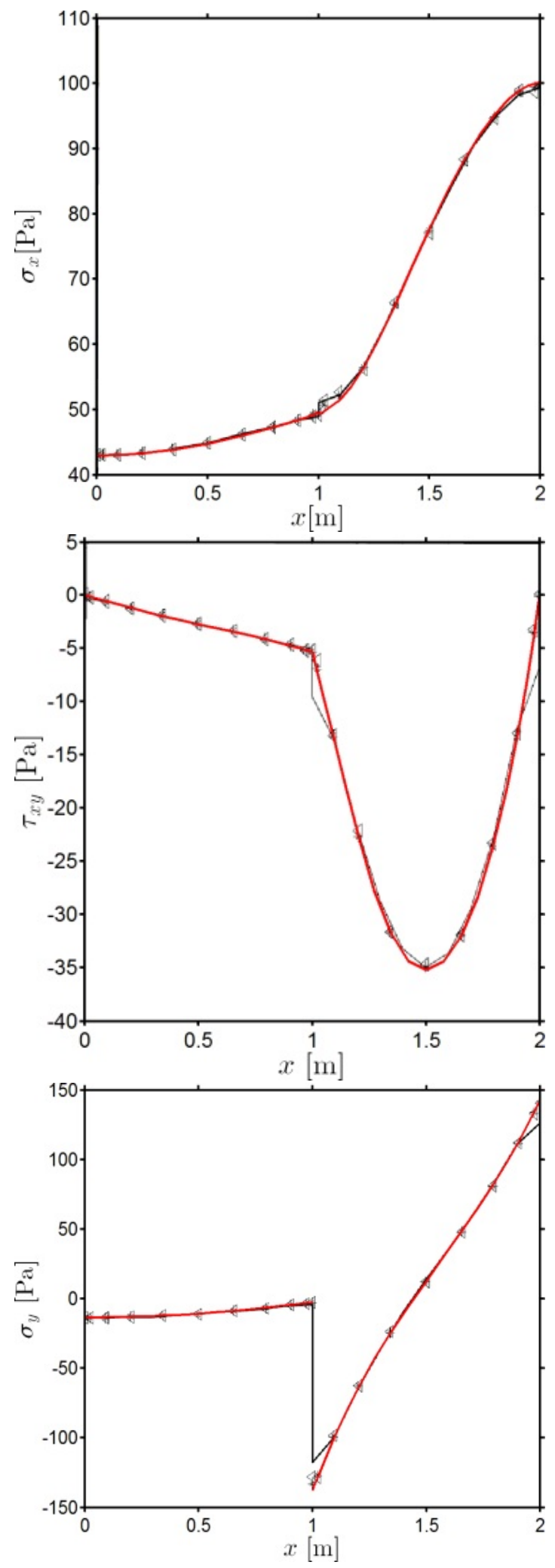

Figure 13: The present result using $N=M=21$ for each element is compared to the same presented by Zong and Zhang [122], who solved the problem using multi-domain DQ and FEM (Abaqus).
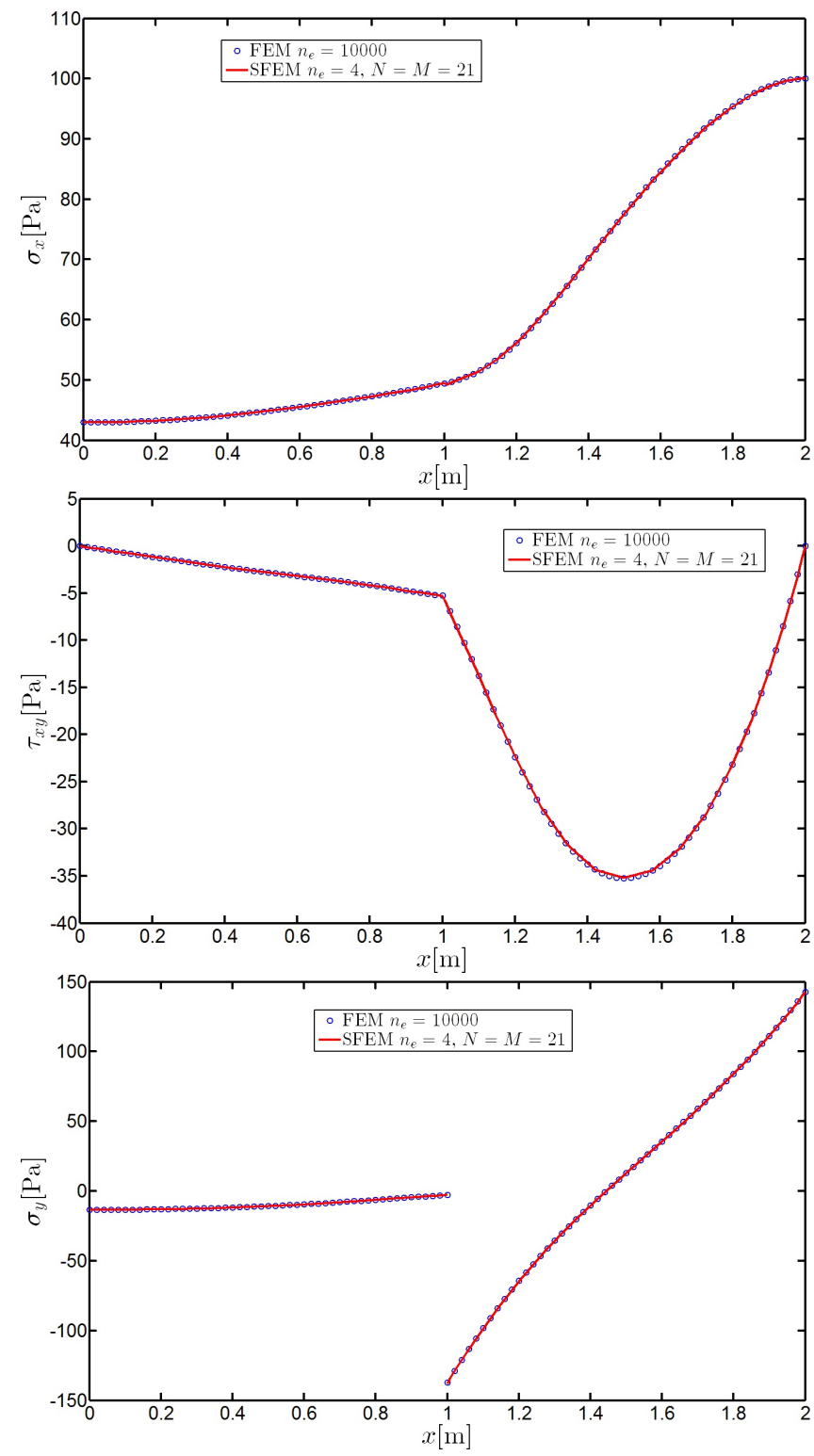

Figure 14: The present result using $N=M=21$ for each element is compared to the same computed by Abaqus.

\subsection{Square plate with a circular inclusion}

Considering the same material data of the previous example a square plate with a circular inclusion is investigated in the following as in Zong and Zhang [122]. The plate is subjected to a vertical traction $q=100 \mathrm{~Pa}$ and a double symmetry occurs in this case also. The quarter plate has $L=2.5 \mathrm{~m}$ and the inclusion has a radius $R=1 \mathrm{~m}$. The mapping technique is compulsory for this case, since distorted elements must be used in order to map the circumference correctly. The plots in Figure 15 use the same symbols of the previous case. For solving this problem four elements are used $n_{e}=4$ with $N=M=21$. The top-left subfigure 
of Figure 15 shows the horizontal displacement $u_{x}$ on the $x$ axis, whereas the others represent the vertical displacement $u_{y}$ and the stresses $\sigma_{x}$ and $\sigma_{y}$ on the yaxis. A very good agreement is observed for the present case also.

\subsection{Vibrations of a 2D cantilever elastic beam}

The problems presented in the present section have been investigated in the past [128-130], when the first ten natural frequencies have been also compared to FEM and other methods $[145,146]$. The same problem is now used to show the convergence of the technique when different number of points are used in each element. It is recalled that the structure is a 2D cantilever beam of length $L=0.1 \mathrm{~m}$ and height $H=0.01 \mathrm{~m}$ with the following mechanical properties $E=205.939 \mathrm{GPa}, v=0.3$ and $\rho=7845.32 \mathrm{~kg} / \mathrm{m}^{3}$. In this section the convergence and stability behaviour of the technique is investigated using a reference solution obtained through commercial FEM code Abaqus. A very fine reference solution is calculated using $n_{e}=10^{5}$ regular (squared) CPS8 (8-node biquadric without reduced integration) elements. Three SFEM structures are drawn using $n_{e}=1, n_{e}=3$ and $n_{e}=10$. It is noted that the first two meshes are made of distorted elements (rectangular shape), whereas the latest has all regular (squared) elements. This choice has been made, because in this way the effect on the mesh distortion can be studied. In particular the mesh distortion can be overcome by using different number of points along two directions.

The convergence error is evaluated in two different ways: using the logarithm of the relative error and the absolute one. The relative error is computed as $\log _{10}\left|f_{1} / f_{1 \text { ref }}-1\right|$, whereas the absolute one as $f_{1}-f_{1 \text { ref }}$. The first measures the difference between the two solutions looking at the absolute value of the significant digits; it is intrinsically dimensionless and lets the user understand the global trend of the solution. The second one just shows the dimensional differences between the two quantities, it is physical so that the user can understand directly the real trend of the solution. It should be noted that the absolute error shows immediately when the trend oscillates towards the minimum error, whereas this aspect is slightly hidden by the relative error due to the imposition of the absolute value. The relative error is a classic way of error measurement in FEM approach, since it is very wellknown that FEM has a 'convergence from above' when the mesh is refined. On the contrary in SFEM, due to the fact that the approximation inside each element can change (as in $p$-FEM), a 'convergence from above' not always occurs.

In Figure 16 the abscissa contains the logarithm of the number of degrees of freedom of each problem dofs $=$ $2(N-2)(M-2) n_{e}$ and the reference value has been taken with respect to a computed eigenvalue of $2.66805 \cdot 10^{7} \rightarrow$ $f_{1 r e f}=822.08573764683945 \mathrm{~Hz}$. It is clear from Figure 16 that when $N \neq M$ and in particular when $N \gg M$ the solution tends to diverge. On the contrary the solution is always stable when $N=M$, but a strong accuracy is reached only when several dofs are considered.

\subsection{Cook isotropic beam with two eccentric holes}

In the present section a new finite element benchmark is proposed, considering the reference problem of Cook's beam [147-149]. The plan-form of the Cook's beam has been kept the same, but two holes have been added in two eccentric locations as depicted in Figure 17a . The elastic material data are $E=3 \cdot 10^{7} \mathrm{~Pa}$ and $v=0.3$ and the shear stress applied at the right side of the plate is equal to $F=100 \mathrm{~Pa}$. The beam is in plane stress conditions. The mesh used in the computation is presented in Figure 17b . The results are presented in tabular and graphical forms. Table 6 reports the comparison in terms of global displacement and Mises stress at a specific point for the static analysis, and the first ten natural frequency for the modal analysis. The reference point for the static analysis has coordinates $(48,52) \mathrm{m}$. All the results are compared to a FEM solution obtained with a relatively fine mesh. Next to each result the percentage of the relative error is presented. As it is obvious, the error decreases when the number of elements increases. It can be noted that the solution can be considered accurate for $N=M=9$ both statically and dynamically. Figure 18 shows a graphical coloured plot of the Mises stress on the whole geometry compared to the same map create with Abaqus software.

\subsection{Laminated composite circular arch with circular holes}

As a final numerical test the free vibration problem of the structure depicted in Figure 19 is described. The arch has an opening angle of 90 degrees and radius $R=2.5 \mathrm{~m}$. The total width of the arch is $h=1 \mathrm{~m}$, with a bottom and top sheets of thickness $h_{s}=0.25 \mathrm{~m}$ and a core with $h_{c}=0.5 \mathrm{~m}$. The holes have a diameter of $d=0.25 \mathrm{~m}$ and they are centred in the core of the arch. There are three holes, one on 


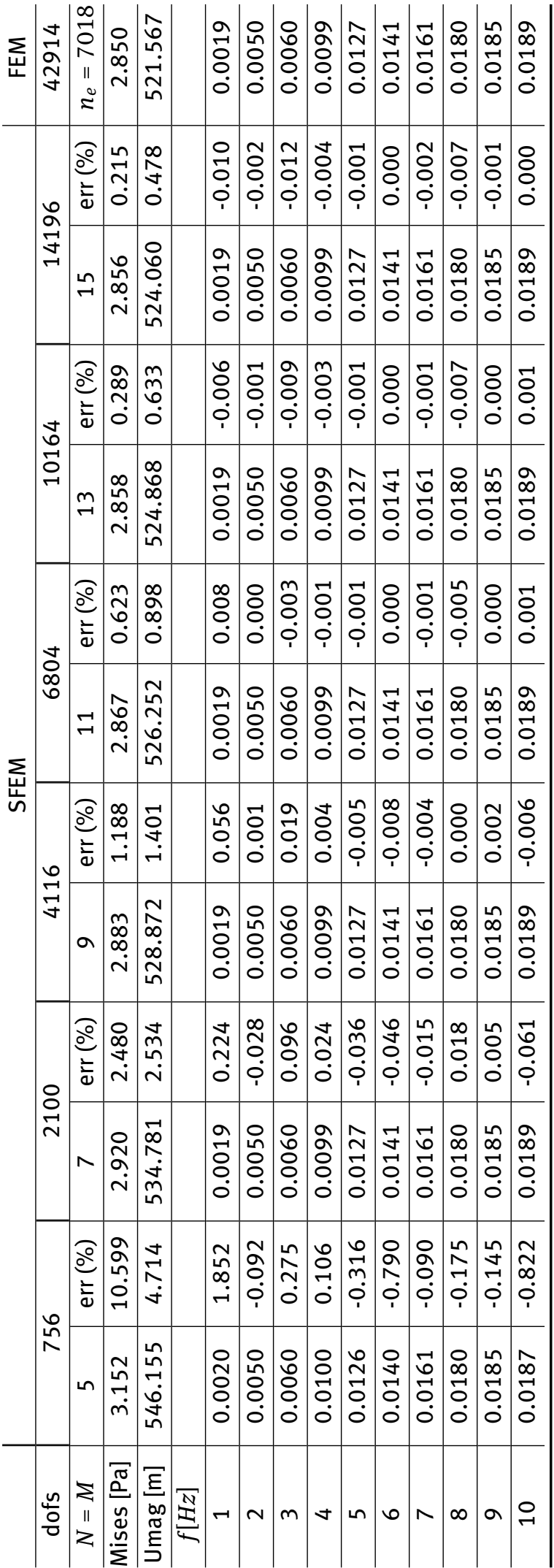



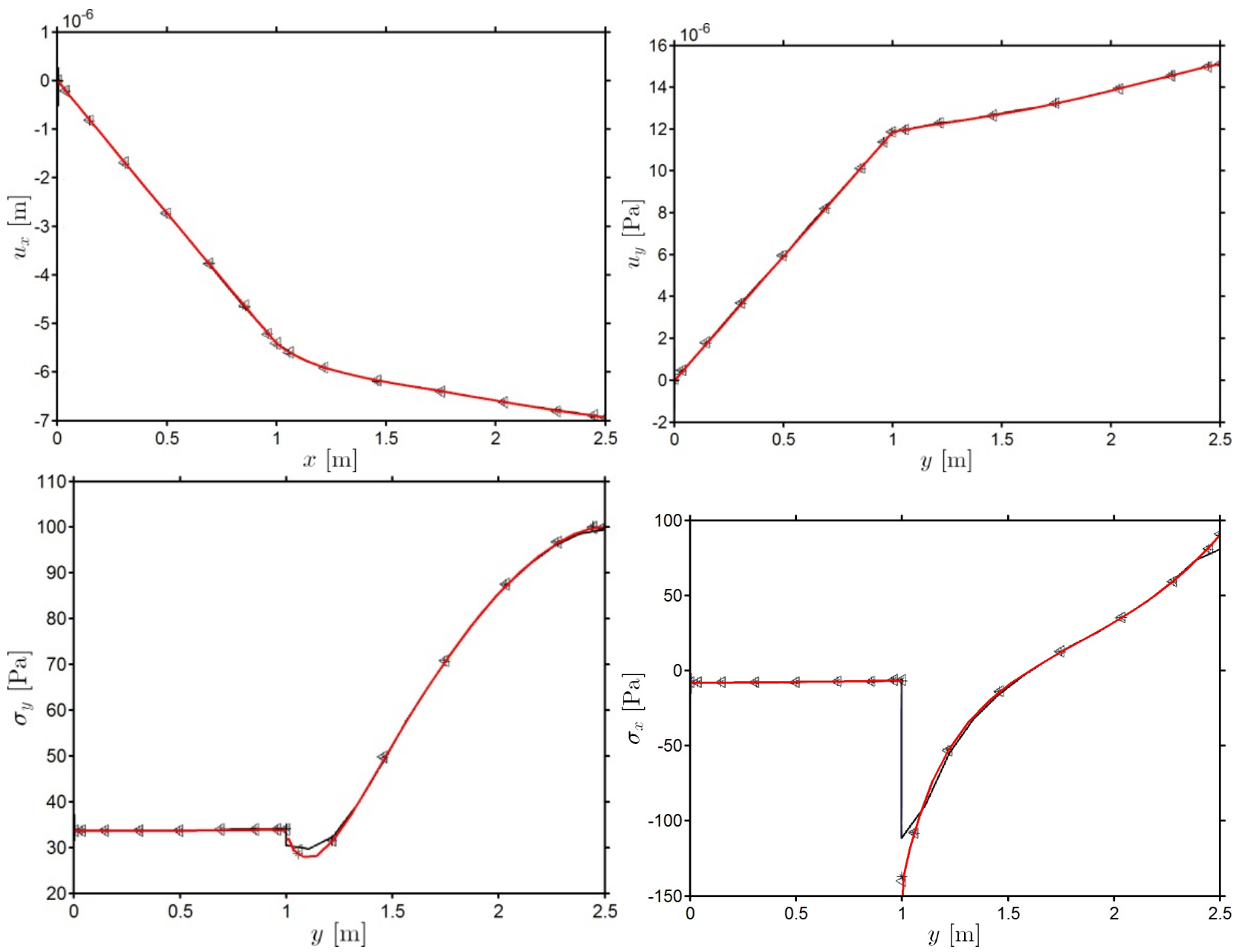

Figure 15: The present result using $N=M=21$ for each element is compared to the same presented by Zong and Zhang [122], who solved the problem using multi-domain DQ and FEM (Abaqus).

the symmetry axis and two half circles are drawn at the boundary edges. Both the right and left edges are clamped (the circular holes are free). The right and left edges are inclined of 45 degrees with respect to the horizontal axis. The arch is in plane stress condition.

The reference numerical solution is carried out using FEM Straus with a relatively fine mesh with 74646 dofs. The SFEM mesh is made of $n_{e}=48$ elements according to Figure 19. Four different solutions are calculated. The first one is referred to an isotropic arch with $E=30 \mathrm{MPa}, v=0.3$ and $\rho=1000 \mathrm{~kg} / \mathrm{m}^{3}$. The others consider laminated structures, when the ratios between the sheets and the core is variable. The elastic modulus of the core is kept constant $E_{c}=E=30 \mathrm{MPa}$ and the Young's modulus of the sheets increases following the ratios $E_{s} / E_{c}=10,100,1000$. Table 7 reports the results in terms of natural frequencies where the readiness is helped with the relative error between the reference FEM solution and the SFEM one. As expected, the error decreases when the number of grid points increases. Furthermore the first six mode shapes of the four cases considered are illustrated in Figures $20-23$. It can be noted that the modes are global, when the structure is isotropic and the ratio between the core and the sheets is small, whereas when the ratio is large the modes concentrate on the soft-core which is the softest part of the structure and the global behaviour is less significant.

\section{Closure}

It can be concluded that the complete set of governing and boundary equations in differential and discrete forms have been presented for elastostatic and elastodynamic problems using the SFEM approach. The present procedure demonstrated to be very accurate to solve both classical and new numerical applications when also composite materials are taken into account. Moreover, some new 

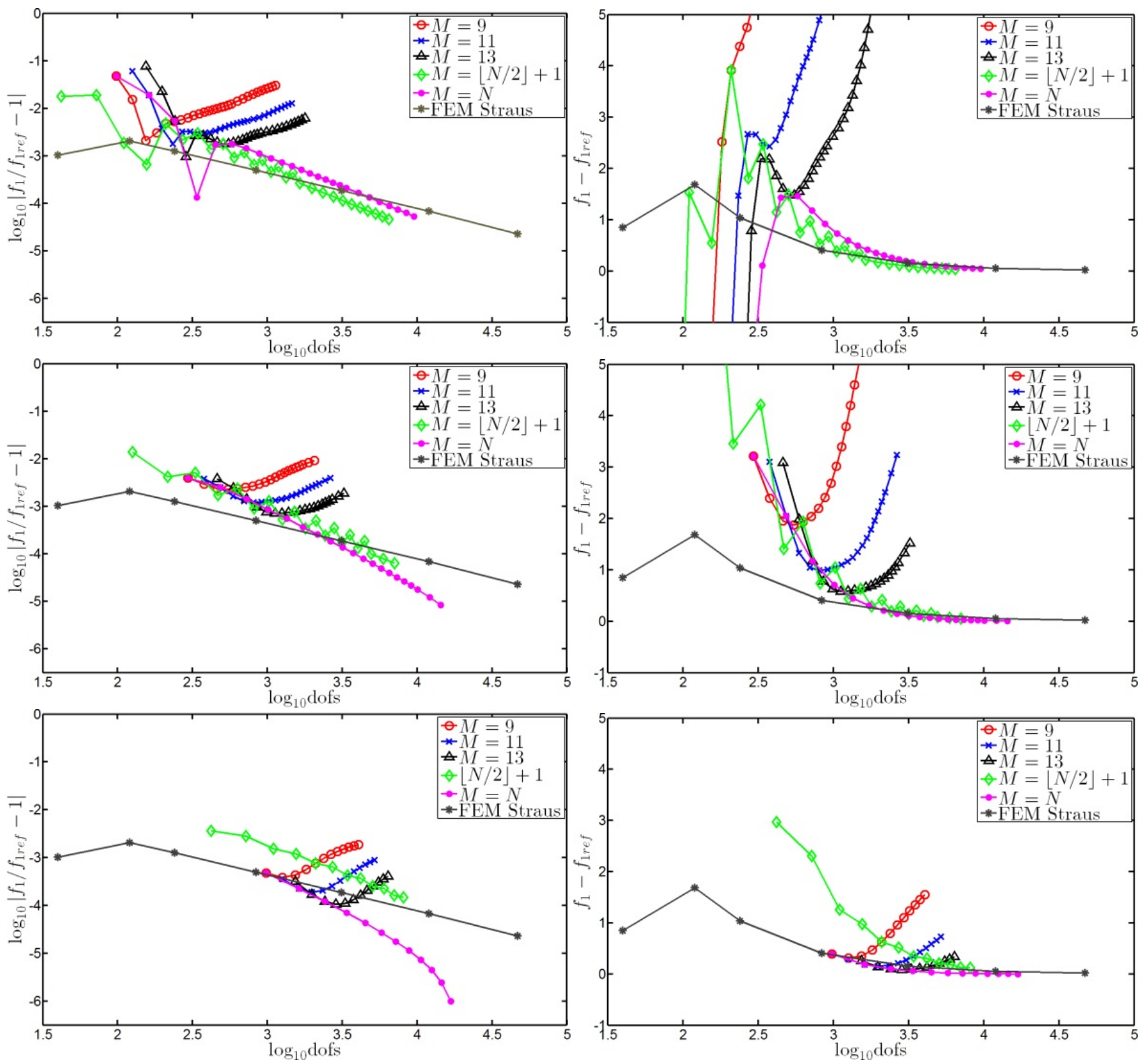

Figure 16: Convergence of the first natural frequency of a cantilever elastic beam with $n_{e}=1, n_{e}=3, n_{e}=10$ and different number of points along the two directions.

numerical benchmarks have been presented for future developments on the same subject. The manuscript deals with the modelling of the present method and the implementation technique of the SFEM. It should be mentioned that the attention is not focused on the stability of the numerical technique when different basis functions and grid distributions are used. Thus, this topic could be the aim of a future paper, where it could be investigated the accuracy and stability of the method when these parameters are changed. The numerical applications provided solutions from the literature and new geometries compared with classic FEM. In all the cases very good agree- ment is observed and this demonstrates the correctness of the methodology, when compared to other classical approaches and FEM commercial codes. Furthermore, following previously published works, the author would like to deepen the knowledge about the application of elastostatic SFEM problems related to fracture mechanics topics [150-153] in future papers.

Acknowledgement: The research topic is one of the subjects of the Centre of Study and Research for the Identification of Materials and Structures (CIMEST)-“M. Capurso" of the University of Bologna (Italy). I am grateful to Erasmo 


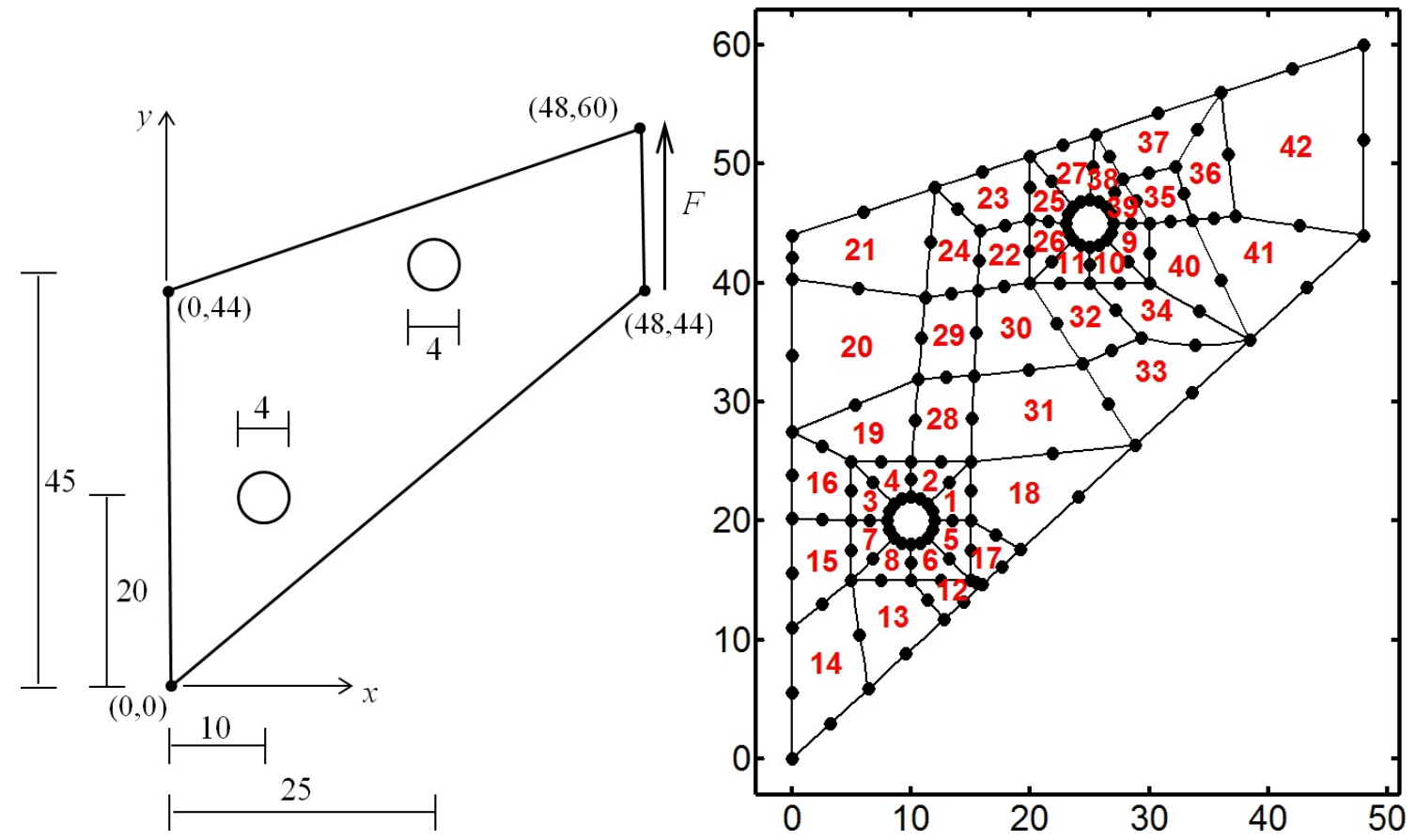

(a)

(b)

Figure 17: a) Geometry of the Cook's beam with two eccentric holes (dimensions in meters [m]). b) SFEM mesh used in the computations.

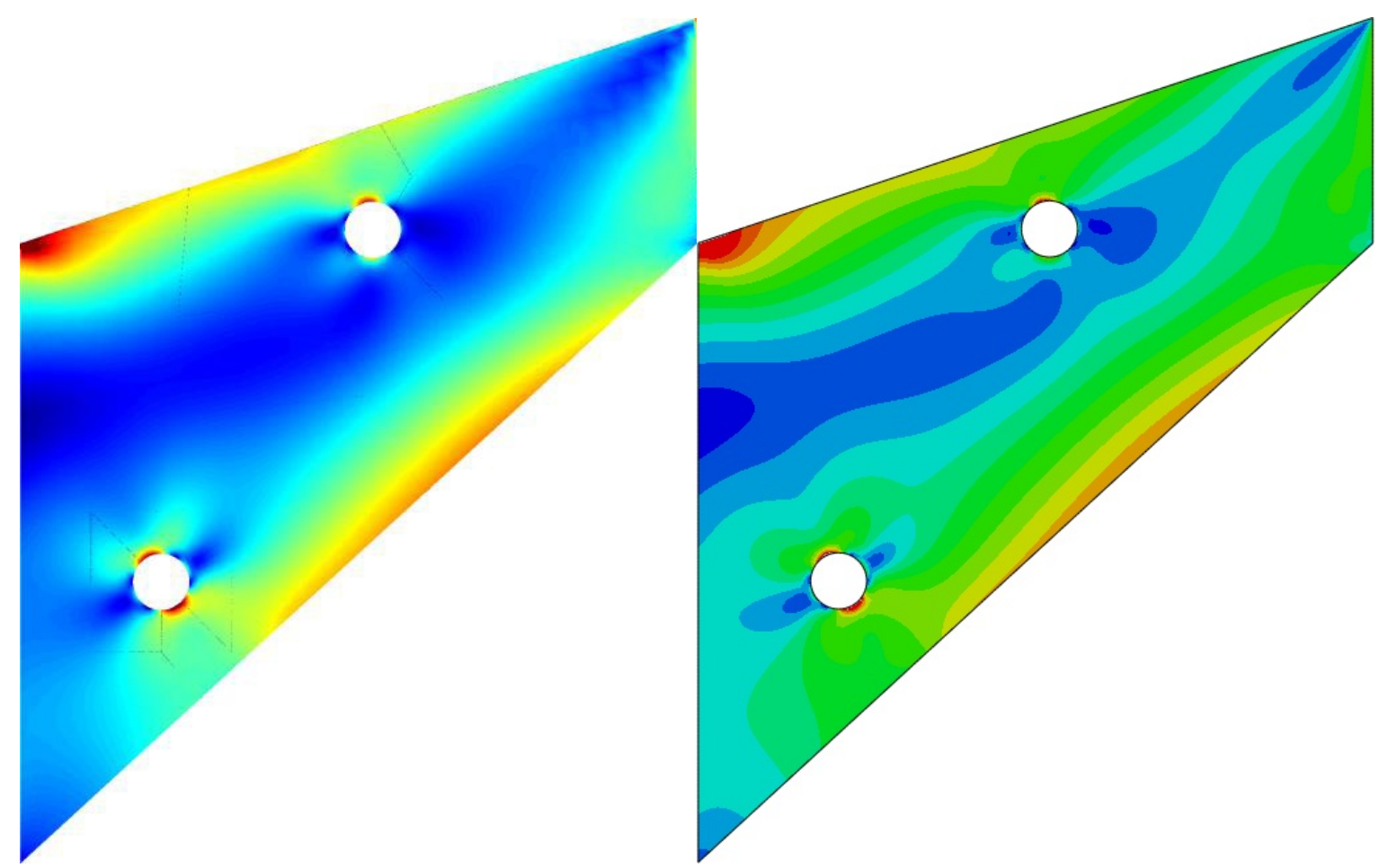

(a)

(b)

Figure 18: a) SFEM Mises stress color map, b) Abaqus FEM Mises color map; both on the undeformed shape. 
Table 7: First ten frequencies for a circular composite arch with circular holes, increasing the number of grid points per element.

\begin{tabular}{|c|c|c|c|c|c|c|c|c|c|}
\hline & M & \multicolumn{8}{|c|}{ SFEM } \\
\hline \multicolumn{10}{|c|}{ Isotropic arch } \\
\hline$[\mathrm{Hz}]$ & $\begin{array}{c}\text { dofs }= \\
=74646\end{array}$ & $\begin{array}{c}n_{e}=48 \\
N=M=5\end{array}$ & err (\%) & $\begin{array}{c}n_{e}=48 \\
N=M=7\end{array}$ & err (\%) & $\begin{array}{c}n_{e}=48 \\
N=M=9\end{array}$ & $\operatorname{rr}(\%)$ & $\begin{array}{c}n_{e}=48 \\
N=M=11\end{array}$ & $\overline{\operatorname{rr}(\%)}$ \\
\hline 1 & 12.28002 & 12.28312 & 0.025 & 12.27989 & -0.001 & 12.28021 & 0.002 & 12.27998 & 0.000 \\
\hline 2 & 5.35798 & 15.36383 & 0.038 & 15.35464 & -0.022 & 15.35973 & 0.011 & 15.35916 & 0.008 \\
\hline 3 & .78326 & 23.80035 & 0.072 & 23.78339 & 0.001 & 23.78345 & 0.001 & 23.78313 & -0.001 \\
\hline 4 & 29.17763 & 29.18699 & 0.032 & 29.17475 & -0.010 & 29.18183 & 0.014 & 29.18046 & 0.010 \\
\hline 5 & 8.81811 & 38.86387 & 0.118 & 38.80420 & -0.036 & 38.82353 & 0.014 & 38.82181 & 0.010 \\
\hline 6 & 0.03110 & 39.88207 & -0.372 & 40.02701 & -0.010 & 40.02956 & -0.004 & 40.03057 & -0.001 \\
\hline 7 & 3.52950 & 53.36088 & -0.315 & 53.51688 & -0.024 & 53.53366 & 0.008 & 53.53343 & 0.007 \\
\hline 8 & .36951 & 57.43483 & 0.114 & 57.36167 & -0.014 & 57.37167 & 0.004 & 57.37036 & 0.001 \\
\hline 9 & .65324 & 60.70018 & 0.077 & 60.65306 & 0.000 & 60.65354 & 000 & 5304 & $\overline{0.000}$ \\
\hline 10 & 63.30650 & 63.21378 & -0.146 & 63.29492 & -0.018 & 63.31129 & 0.008 & 1032 & 0.006 \\
\hline \multicolumn{10}{|c|}{ Composite $\operatorname{arch} E / E_{c}=10$} \\
\hline 1 & .39486 & 26.36465 & -0.114 & 26.39481 & 0.000 & 26.39486 & 0.000 & 26.39478 & 0.000 \\
\hline 2 & 26.93003 & 26.99436 & 0.239 & 26.94819 & 0.067 & 26.93976 & 0.036 & 26.93453 & 0.017 \\
\hline 3 & .24074 & 50.21545 & -0.050 & 50.27271 & 0.064 & 50.25536 & 029 & 0.24771 & 0.014 \\
\hline 4 & 1.76582 & 51.77008 & 0.008 & 51.77039 & 0.009 & 51.76707 & 0.002 & 1.76588 & 0.000 \\
\hline 5 & 66.56456 & 66.59229 & 0.042 & 66.58919 & 0.037 & 66.58092 & 0.025 & 66.57215 & 0.011 \\
\hline 6 & 74.78007 & 74.31327 & -0.624 & 74.77522 & -0.006 & 74.77924 & -0.001 & 3089 & 001 \\
\hline 7 & 82.17393 & 82.13006 & -0.053 & 82.17678 & 0.003 & 82.17621 & 0.003 & 82.17474 & 0.001 \\
\hline 8 & .01456 & 83.82778 & -0.222 & 84.03978 & 0.030 & 2784 & 016 & 2101 & 0.008 \\
\hline 9 & 90.81161 & 90.75894 & -0.058 & 90.81334 & 0.002 & 90.81344 & 002 & 1228 & 0.001 \\
\hline 10 & .57337 & 97.25551 & -0.326 & 97.57839 & 0.005 & 97.57610 & 0.003 & 7478 & 0.001 \\
\hline \multicolumn{10}{|c|}{ Composite $\operatorname{arch} E / E_{c}=100$} \\
\hline 1 & .92372 & 53.09493 & 0.323 & 52.99118 & 0.127 & 52.95069 & 0.051 & 52.93544 & 0.022 \\
\hline 2 & .73952 & 64.72375 & -0.024 & 64.74849 & 0.014 & 64.74164 & 0.003 & 64.73995 & 0.001 \\
\hline 3 & 92.17044 & 92.03309 & -0.149 & 92.22634 & 0.061 & 92.19096 & 0.022 & 92.17969 & 0.01 \\
\hline 4 & 1.1039 & 100.9813 & -0.121 & 101.1082 & 0.004 & 1075 & 004 & 1055 & 0.002 \\
\hline 5 & 3.1860 & 103.0722 & -0.110 & 103.2130 & 0.026 & 103.1994 & 0.013 & 103.1920 & 0.006 \\
\hline 6 & 108.9535 & 108.6645 & -0.265 & 108.9564 & 0.003 & 108.9577 & 0.004 & 108.9560 & 0.002 \\
\hline 7 & 122.7692 & 122.4327 & -0.274 & 122.7823 & 0.011 & 122.7800 & 0.009 & 122.7749 & 0.005 \\
\hline 8 & 7.7322 & 127.4390 & -0.230 & 127.7277 & -0.004 & 127.7340 & 0.001 & 127.7330 & 0.001 \\
\hline 9 & 128.5981 & 128.1156 & -0.375 & 128.6563 & 0.045 & 6226 & 019 & .6095 & 0.009 \\
\hline 10 & 3.6918 & 133.3974 & -0.220 & 133.6987 & 0.005 & 133.7026 & 0.008 & 133.6980 & 0.005 \\
\hline \multicolumn{10}{|c|}{ Composite $\operatorname{arch} E / E_{c}=1000$} \\
\hline 1 & 105.7750 & 105.7615 & -0.013 & 105.8164 & 0.039 & 105.7919 & 0.016 & 105.7829 & 0.007 \\
\hline 2 & 0.1931 & 110.0682 & -0.113 & 110.1940 & 0.001 & 110.1935 & 0.000 & 110.1935 & 0.000 \\
\hline 3 & 116.8438 & 116.7519 & -0.079 & 116.8677 & 0.020 & 116.8522 & 0.007 & 116.848 & 0.004 \\
\hline 4 & 126.3414 & 125.9453 & -0.314 & 126.3211 & -0.016 & 126.3378 & -0.003 & 126.3409 & 0.000 \\
\hline 5 & 137.8245 & 137.4203 & -0.293 & 137.8758 & 0.037 & 137.8512 & 0.019 & 137.8364 & 0.009 \\
\hline 6 & 150.4189 & 149.6223 & -0.530 & 150.3782 & -0.027 & 150.4160 & -0.002 & 150.4190 & 0.000 \\
\hline 7 & 150.7741 & 150.2760 & -0.330 & 150.7483 & -0.017 & 150.7715 & -0.002 & 150.7744 & 0.000 \\
\hline 8 & 155.8039 & 155.7738 & -0.019 & 155.8231 & 0.012 & 155.8049 & 0.001 & 155.8037 & 0.000 \\
\hline 9 & 172.5842 & 172.3676 & -0.126 & 172.6097 & 0.015 & 172.5949 & 0.006 & 172.5893 & 0.003 \\
\hline 10 & 177.8687 & 177.4118 & -0.257 & 177.8791 & 0.006 & 177.8712 & 0.001 & 177.8700 & 0.001 \\
\hline
\end{tabular}




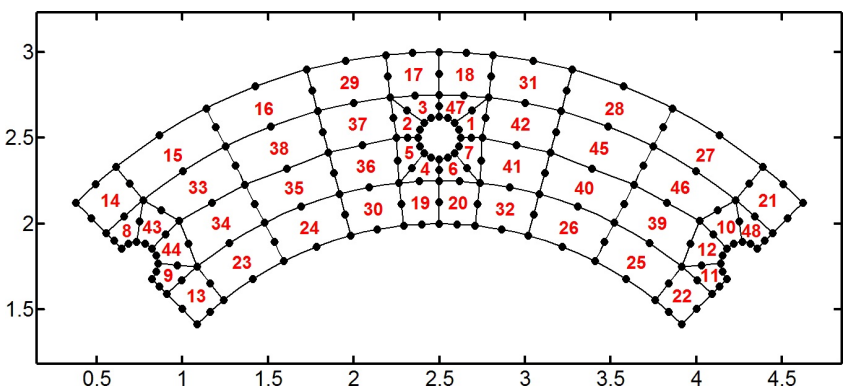

Figure 19: SFEM mesh of a laminated composite circular arch with circular holes.

Viola and Francesco Tornabene for their encouragement throughout the preparation of this work. A special thanks goes to Francesco Tornabene who pushed me to improve the manuscript day after day.

\section{References}

[1] Love A.E.H., A Treatise on the Mathematical Theory of Elasticity, Dover, 1944.

[2] Timoshenko S., Goodier J.N., Theory of Elasticity, McGraw-Hill, 1951.

[3] Sokolnikoff I.S., Mathematical Theory of Elasticity, McGrawHill, 1956.

[4] Lekhnitskii S.G., Theory of Elasticity of an Anisotropic Body, Mir Publishers, 1981.

[5] Kaw A.K., Mechanics of Composite Materials, CRC Press, 1997.

[6] Mase G.T., Mase G.E., Continuum Mechanics for Engineers, CRC Press, 1999.

[7] Jones R.M., Mechanics of Composite Materials, Taylor \& Francis, 1999.

[8] Reddy J.N., Energy Principles and Variational Methods in Applied Mechanics, John Wiley \& Sons, 2002.

[9] Wempner G., Talaslidis D., Mechanics of Solids and Shells, CRC Press, 2003.

[10] Leissa A.W., Qatu M.S., Vibrations of Continuous Systems, McGraw-Hill, 2011.

[11] Bellman R., Casti J., Differential quadrature and long-term integration, J. Math. Anal. Appl., 1971, 34, 235-238.

[12] Bellman R., Kashef B.G., Casti J., Differential quadrature: A technique for the rapid solution of nonlinear partial differential equations, J. Comput. Phys., 1972, 10, 40-52.

[13] Finlayson B.A., Scriven L.E., The method of weighted residual: a review, Appl. Mech. Rev., 1966, 19, 735-748.

[14] Gottlieb D., Orszag S.A., Numerical analysis of spectral methods. Theory and applications, CBMS-NSF Regional Conf. Ser. In Appl. Math., SIAM, 1977.

[15] Boyd J.P., Chebyshev and Fourier spectral methods, Dover Publications, 2001.

[16] Quan J.R., A unified approach for solving nonlinear partial differential equations in chemical engineering applications, Master thesis, University of Nebraska-Lincoln.
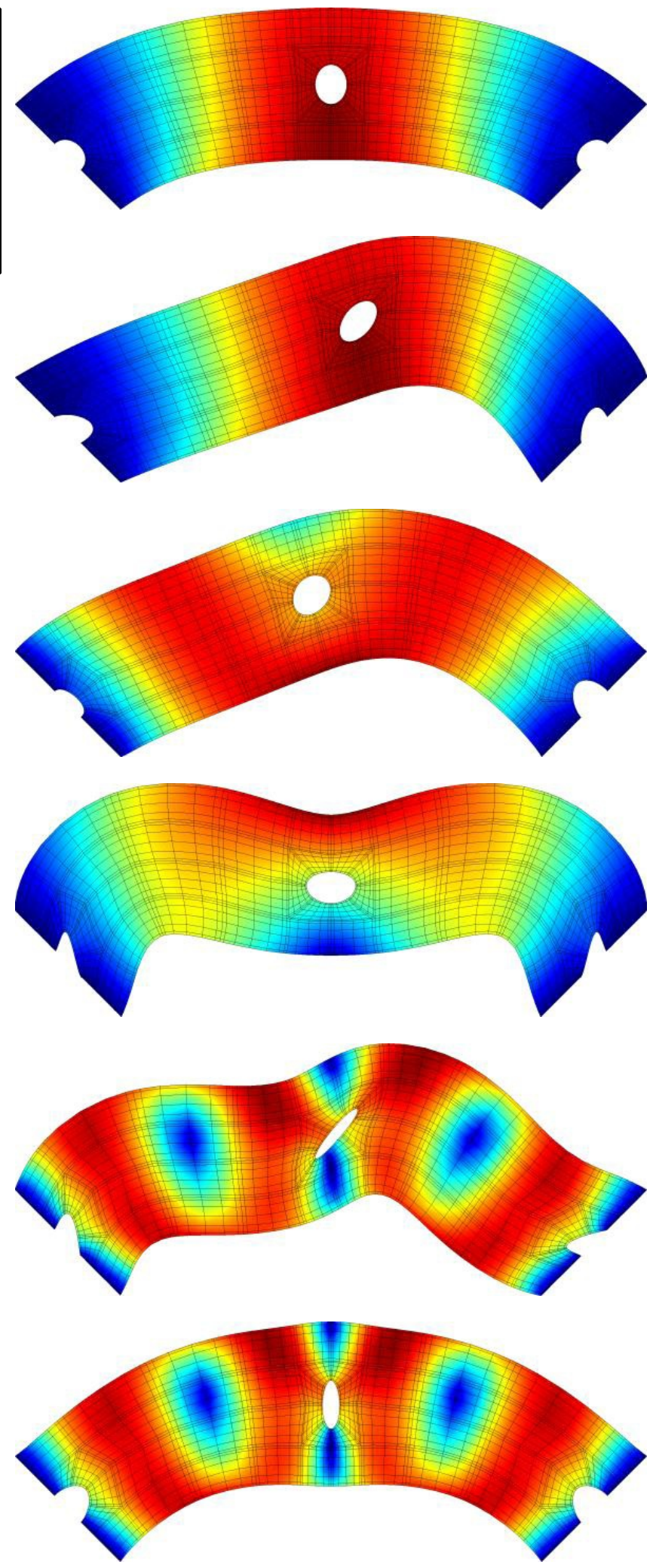

Figure 20: First six mode shapes of an isotropic arch using $N=M=$ 7 grid per element. 

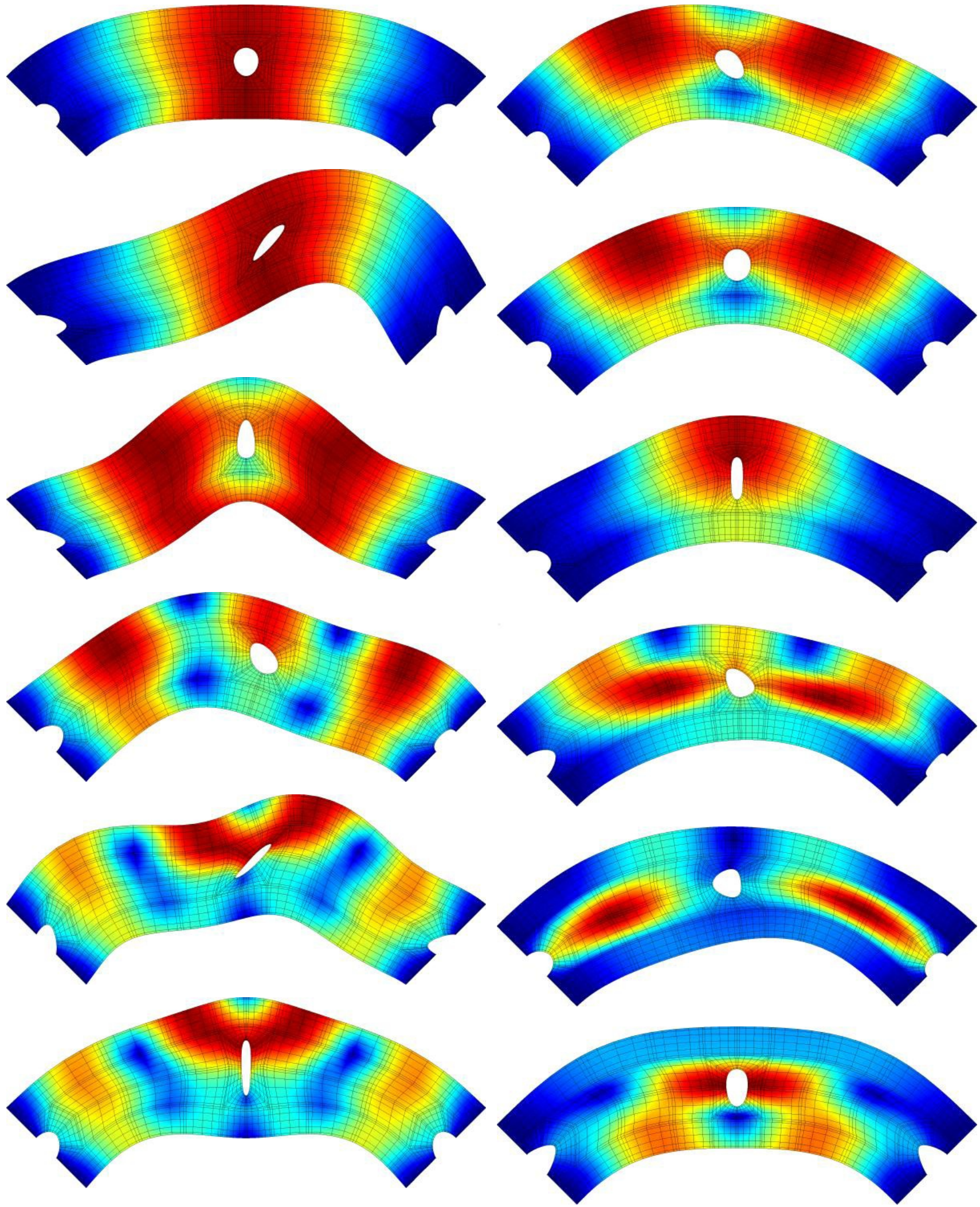

Figure 21: First six mode shapes of a composite arch with ratio $E_{s} / E_{c}=10$ using $N=M=7$ grid per element.

Figure 22: First six mode shapes of a composite arch with ratio $E_{s} / E_{c}=100$ using $N=M=7$ grid per element. 

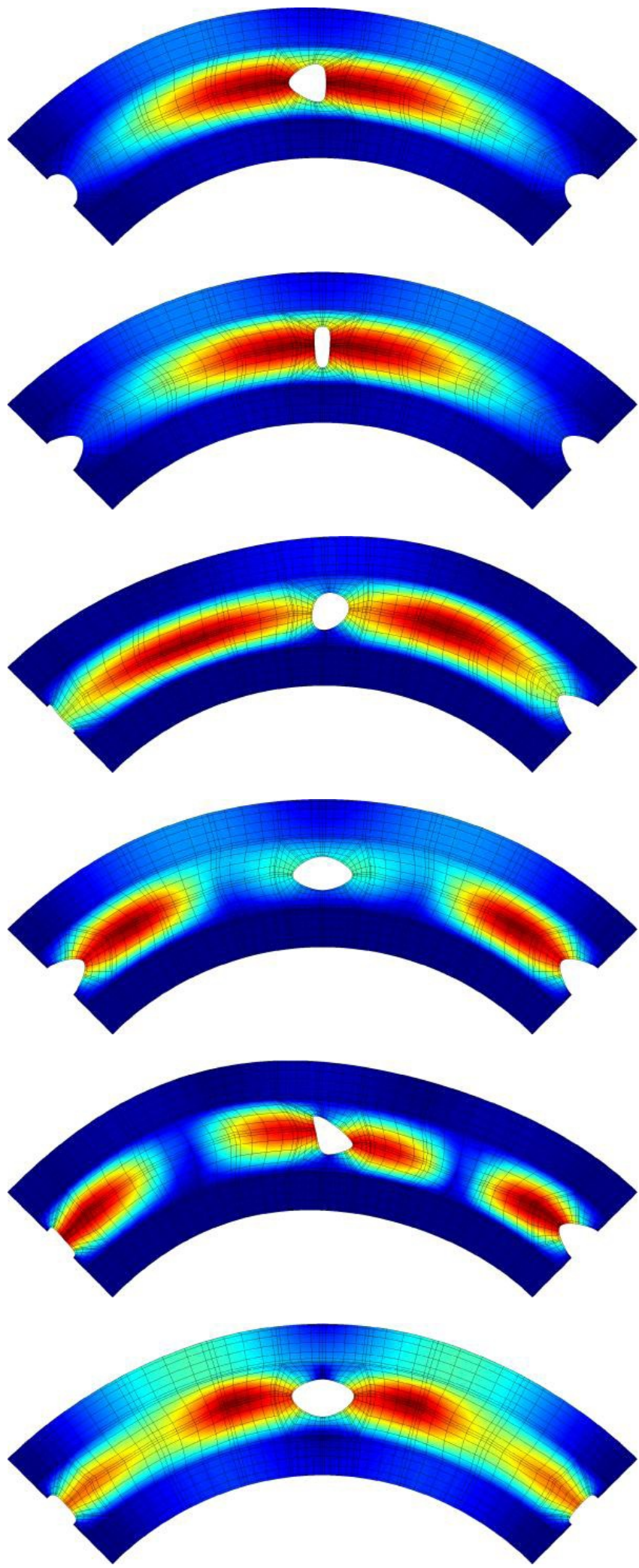

Figure 23: First six mode shapes of a composite arch with ratio $E_{S} / E_{c}=1000$ using $N=M=7$ grid per element.
[17] Quan J.R., Chang C.T., New insights in solving distributed system equations by the quadrature method - I. Analysis, Comput. Chem. Eng., 1989, 13, 779-788.

[18] Quan J.R., Chang C.T., New insights in solving distributed system equations by the quadrature method - II. Numerical experiments, Comput. Chem. Eng., 1989, 13, 1017-1024.

[19] Bert C.W., Malik M., Differential quadrature method in computational mechanics, Appl. Mech. Rev., 1996, 49, 1-27.

[20] Shu C., Generalized differential-integral quadrature and application to the simulation of incompressible viscous flows including parallel computation, PhD Thesis, University of Glasgow, UK, 1991.

[21] Shu C., Richards B.E., Parallel simulation of incompressible viscous flows by generalized differential quadrature, Comput. Syst. Eng., 1992, 3, 271-281.

[22] Shu C., Richards B.E., Application of generalized differential quadrature to solve two-dimensional incompressible NavierStokes equations, Int. J. Numer. Meth. Fluids, 1992, 15, 791798.

[23] Bert C.W., Jang S.K., Striz A.G., Two new approximate methods for analyzing free vibration of structural components, AIAA J., 1988, 26, 612-618.

[24] Bert C.W., Jang S.K., Striz, A.G., Nonlinear bending analysis of orthotropic rectangular plates by the method of differential quadrature, Comput. Mech., 1989, 5, 217-226.

[25] Bert C.W., Malik M., Free vibration analysis of thin cylindrical shells by the differential quadrature method, J. Press. Vess. Technol., 1996, 118, 1-12.

[26] Bert C.W., Wang X., Striz A.G., Differential quadrature for static and free vibration analyses of anisotropic plates, Int. J. Solids Struct., 1993, 30, 1737- 1744.

[27] Bert C.W., Wang X., Striz A.G., Static and free vibrational analysis of beams and plates by differential quadrature method, Acta Mech., 1994, 102, 11-24.

[28] Bert C.W., Wang X., Striz A.G., Convergence of DQ method in the analysis of anisotropic plates, J. Sound Vib., 1994, 170, 140-144.

[29] Jang S.K., Application of Differential Quadrature to the Analysis of Structural Components, PhD Thesis, University of Oklahoma, 1987.

[30] Jang S.K., Bert C.W., Striz A.G., Application of differential quadrature to static analysis of structural components, Int. J. Numer. Meth. Engng., 1989, 28, 561-577.

[31] Kang K., Bert C.W., Striz A.G., Vibration analysis of shear deformable circular arches by the differential quadrature method, J. Sound Vib., 1995, 181, 353-360.

[32] Kang K.J., Bert C.W. Striz, A.G., Vibration and buckling analysis of circular arches using DQM, Comput. Struct., 1996, 60, 49-57.

[33] Shu C., Free vibration analysis of composite laminated conical shells by generalized differential quadrature, J. Sound Vib., 1996, 194, 587-604.

[34] Shu C., An efficient approach for free vibration analysis of conical shells, Int. J. Mech. Sci., 1996, 38, 935-949.

[35] Shu C., Du H., Implementation of clamped and simply supported boundary conditions in the GDQ free vibration analysis of beams and plates, Int. J. Solids Struct., 1997, 34, 819-835.

[36] Shu C., Wang C.M., Treatment of mixed and nonuniform boundary conditions in GDQ vibration analysis of rectangular plates, Eng. Struct., 1999, 21, 125-134. 
[37] Ng T.Y., Li H., Lam K.Y., Loy C.T., Parametric instability of conical shells by the generalized differential quadrature method, Int. J. Numer. Meth. Engng., 1999, 44, 819-837.

[38] Ng T.Y., Lam K.Y., Free vibration analysis of rotating circular cylindrical shells on elastic foundation, J. Vib. Acoust., 2000, 122, 85-89.

[39] Lam K.Y., Hua L., Influence of initial pressure on frequency characteristics of a rotating truncated circular conical shell, Int. J. Mech. Sci., 2000, 42, 213-236.

[40] Shu C., Differential Quadrature and Its Application in Engineering, Springer, 2000.

[41] Lam K.Y., Li H., Ng T.Y., Chua C.F., Generalized differential quadrature method for the free vibration of truncated conical panels, J. Sound Vib., 2002, 251, 329-348.

[42] Ng T.Y., Li H., Lam K.Y., Generalized differential quadrature method for the free vibration of rotating composite laminated conical shell with various boundary conditions, Int. J. Mech. Sci., 2003, 45, 567-587.

[43] Ng C.H.W., Zhao Y.B., Wei G.W., Comparison of discrete convolution and generalized differential quadrature for the vibration analysis of rectangular plates, Comput. Meth. Appl. Mech. Engng., 2004, 193, 2483-2506.

[44] Civalek Ö., Application of differential quadrature (DQ) and harmonic differential quadrature (HDQ) for buckling analysis of thin isotropic plates and elastic columns, Eng. Struct., 2004, 26, 171-186.

[45] Civalek Ö., Geometrically nonlinear analysis of doubly curved isotropic shells resting on elastic foundation by a combination of harmonic differential quadrature-finite difference methods, Int. J. Pres. Ves. Pip., 2005, 82, 470-479.

[46] Viola E., Tornabene F., Vibration analysis of damaged circular arches with varying cross-section, Struct. Integr. Durab. (SIDSDHM), 2005, 1, 155-169.

[47] Viola E., Tornabene F., Vibration analysis of conical shell structures using GDQ method, Far East J. Appl. Math., 2006, 25, 2339.

[48] Tornabene F., Modellazione e Soluzione di Strutture a Guscio in Materiale Anisotropo, PhD Thesis, University of Bologna, Italy, 2007.

[49] Tornabene F., Viola E., Vibration analysis of spherical structural elements using the GDQ method, Comput. Math. Appl., 2007, 53, 1538-1560.

[50] Viola E., Dilena M., Tornabene F., Analytical and numerical results for vibration analysis of multi-stepped and multidamaged circular arches, J. Sound Vib., 2007, 299, 143-163.

[51] Marzani A., Tornabene F., Viola E., Nonconservative stability problems via generalized differential quadrature method, J. Sound Vib. 315, 2008, 176-196.

[52] Tornabene F., Viola E., 2-D solution for free vibrations of parabolic shells using generalized differential quadrature method, Eur. J. Mech. A-Solid, 2008, 27, 1001-1025.

[53] Tornabene F., Free vibration analysis of functionally graded conical, cylindrical and annular shell structures with a fourparameter power-law distribution, Comput. Methods Appl. Mech. Engrg., 2009, 198, 2911-2935.

[54] Tornabene F., Viola E., Free vibrations of four-parameter functionally graded parabolic panels and shell of revolution, Eur. J. Mech. A-Solid, 2009, 28, 991-1013.

[55] Tornabene F., Viola E., Free vibration analysis of functionally graded panels and shells of revolution, Meccanica, 2009, 44,
255-281.

[56] Hong C.C., Thermal bending analysis of shear-deformable laminated anisotropic plates by the GDQ method, Mech. Res. Commun., 2009, 36, 804-810.

[57] Tornabene F., Viola E., Inman D.J., 2-D differential quadrature solution for vibration analysis of functionally graded conical, cylindrical and annular shell structures, J. Sound Vib., 2009, 328, 259-290.

[58] Viola E., Tornabene F., Free vibrations of three parameter functionally graded parabolic panels of revolution, Mech. Res. Commun., 2009, 36, 587-594.

[59] Sadeghian H., Rezazadeh G., Comparison of generalized differential quadrature and Galerkin methods for the analysis of micro-electro-mechanical coupled systems, Communications in Nonlinear Science and Numerical Simulation, 2009, 14, 2807-2816.

[60] Tornabene F., Marzani A., Viola E., Elishakoff I., Critical flow speeds of pipes conveying fluid by the generalized differential quadrature method, Adv. Theor. Appl. Mech., 2010, 3, 121-138.

[61] Tornabene F., 2-D GDQ solution for free vibrations of anisotropic doubly-curved shells and panels of revolution, Compos. Struct., 2011, 93, 1854-1876.

[62] Tornabene F., Free vibrations of anisotropic doubly-curved shells and panels of revolution with a free-form meridian resting on Winkler-Pasternak elastic foundations, Compos. Struct., 2011, 94, 186-206.

[63] Tornabene F., Liverani A., Caligiana G., FGM and laminated doubly-curved shells and panels of revolution with a free-form meridian: a 2-D GDQ solution for free vibrations, Int. J. Mech. Sci., 2011, 53, 446-470.

[64] Viola E., Rossetti L., Fantuzzi N., Numerical investigation of functionally graded cylindrical shells and panels using the generalized unconstrained third order theory coupled with the stress recovery, Compos. Struct., 2012, 94, 3736-3758.

[65] Tornabene F., Liverani A., Caligiana G., Laminated composite rectangular and annular plates: a GDQ solution for static analysis with a posteriori shear and normal stress recovery, Compos. Part B-Eng., 2012, 43, 1847-1872.

[66] Tornabene F., Liverani A., Caligiana G., Static analysis of laminated composite curved shells and panels of revolution with a posteriori shear and normal stress recovery using generalized differential quadrature method, Int. J. Mech. Sci., 2012, 61, 7187.

[67] Tornabene F., Liverani A., Caligiana G., General anisotropic doubly-curved shell theory: a differential quadrature solution for free vibrations of shells and panels of revolution with a freeform meridian, J. Sound Vib., 2012, 331, 4848-4869.

[68] Ferreira A.J.M., Viola E., Tornabene F., Fantuzzi N., Zenkour A.M., Analysis of sandwich plates by generalized differential quadrature method, Math. Probl. Eng., 2013, 2013, 1-22, Article ID 964367.

[69] Tornabene F., Ceruti A., Free-form laminated doubly-curved shells and panels of revolution resting on Winkler-Pasternak elastic foundations: a 2-D GDQ solution for static and free vibration analysis, World J. Mech., 2013, 3, 1-25.

[70] Tornabene F., Reddy J.N., FGM and laminated doubly-curved and degenerate shells resting on nonlinear elastic foundations: a GDQ solution for static analysis with a posteriori stress and strain recovery, J. Indian Inst. Sci., 2013, 93, 635-688. 
[71] Tornabene F., Viola E., Static analysis of functionally graded doubly-curved shells and panels of revolution, Meccanica, 2013, 48, 901-930.

[72] Tornabene F., Viola E., Fantuzzi N., General higher-order equivalent single layer theory for free vibrations of doubly-curved laminated composite shells and panels, Compos. Struct., 2013, 104, 94-117.

[73] Viola E., Tornabene F., Fantuzzi N., General higher-order shear deformation theories for the free vibration analysis of completely doubly-curved laminated shells and panels, Compos. Struct., 2013, 95, 639-666.

[74] Viola E., Tornabene F., Fantuzzi N., Static analysis of completely doubly-curved laminated shells and panels using general higher-order shear deformation theories, Compos. Struct., 2013, 101, 59-93.

[75] Tornabene F., Fantuzzi N., Mechanics of Laminated Composite Doubly-Curved Shell Structures. The Generalized Differential Quadrature Method and the Strong Formulation Finite Element Method, Esculapio, 2014.

[76] Ferreira A.J.M., Carrera E., Cinefra M., Viola E., Tornabene F., Fantuzzi N., Zenkour A.M., Analysis of thick isotropic and cross-ply laminated plates by generalized differential quadrature method and a unified formulation, Compos. Part B-Eng., 2014, 58, 544-552.

[77] Tornabene F., Fantuzzi N., Viola E., Carrera E., Static analysis of doubly-curved anisotropic shells and panels using CUF approach, differential geometry and differential quadrature method, Compos. Struct., 2014, 107, 675-697.

[78] Tornabene F., Fantuzzi N., Viola E., Reddy J.N., WinklerPasternak foundation effect on the static and dynamic analyses of laminated doubly-curved and degenerate shells and panels, Compos. Part B-Eng., 2014, 57, 269-296.

[79] Tornabene F., Ceruti, A., Mixed static and dynamic optimization of four-parameter functionally graded completely doublycurved and degenerate shells and panels using GDQ method, Math. Probl. Eng., 2013, 2013, 1-33, Article ID 867079.

[80] Viola E., Rossetti L., Fantuzzi N., Tornabene F., Static analysis of functionally graded conical shells and panels using the generalized unconstrained third order theory coupled with the stress recovery, Compos. Struct., 2014, 112, 44-65.

[81] Tornabene F., Fantuzzi N., Bacciocchi M., The local GDQ method applied to general higher-order theories of doublycurved laminated composite shells and panels: the free vibration analysis, Compos. Struct., 2014, 116, 637-660.

[82] Tornabene F., Fantuzzi N., Bacciocchi M., Free vibrations of free-form doubly-curved shells made of functionally graded materials using higher-order equivalent single layer theories, Compos. Part B Eng., 2014, 67, 490-509.

[83] Viola E., Tornabene F., Fantuzzi N., Stress and strain recovery of laminated composite doubly-curved shells and panels using higher-order formulations, Key Eng. Mat., 2015, 624, 205-213.

[84] Tornabene F., Fantuzzi N., Viola E., Batra R.C., Stress and strain recovery for functionally graded free-form and doubly-curved sandwich shells using higher-order equivalent single layer theory, Compos. Struct., 2015, 119, 67-89.

[85] Liu G.R., Mesh Free Methods. Moving Beyond the Finite Element Method, CRC Press, 2003.

[86] Li H., Mulay S.S., Meshless Methods and their Numerical Properties, CRC Press, 2013.
[87] Liew K.M., Huang Y.Q., Reddy J.N., Analysis of general shaped thin plates by the moving least-squares differential quadrature method, Finite Elem. Anal. Design, 2004, 40, 1453-1474.

[88] Li Q.S., Huang Y.Q., Moving least-squares differential quadrature method for free vibration of antisymmetric laminates, J. Eng. Mech., 2004, 130, 1447-1457.

[89] Huang Y.Q., Li Q.S., Bending and buckling analysis of antisymmetric laminates using the moving least square differential quadrature method, Comput. Meth. Appl. Mech. Engng., 2004, 193, 3471-3492.

[90] Lanhe W., Hua L., Daobin W., Vibration analysis of generally laminated composite plates by the moving least squares differential quadrature method, Compos. Struct., 2005, 68, 319330.

[91] Sator L., Sladek V., Sladek J., Coupling effects in elastic analysis of FGM composite plates by mesh-free methods", Compos. Struct., 2014, 115, 100-110.

[92] Sator L., Sladek V., Sladek J., Analysis of Beams with Transversal Gradations of the Young's Modulus and Variable Depths by the Meshless Method, Slovak J. Civil Eng., 2014, 22, 23-36.

[93] Belytschko T., Lu Y.Y., Gu L., Element-free Galerkin methods, Int. J. Numer. Meth. Engng., 1994, 37, 229-256.

[94] Belytschko T., Lu Y.Y., Gu L., Tabbara M., Element-free Galerkin methods for static and dynamic fracture, Int. J. Solids Struct., 1995, 32, 2547- 2570.

[95] Belytschko T., Krongauz Organ D., Fleming M., Krysl P., Meshless methods: an overview and recent developments, Comput. Meth. Appl. Engng., 1996, 139, 3-47.

[96] Krysl P., Belytschko T., Element-free Galerkin method: convergence, Comput. Meth. Appl. Mech. Engng., 1997, 148, 257-277.

[97] Atluri S.N., Zhu T., A new Meshless Local Petrov-Galerkin (MLPG) approach to nonlinear problems in computer modeling \& simulation, Comput. Model. Simul. Eng., 1998, 3, 187-196.

[98] Atluri S.N., Zhu T., A new Meshless Local Petrov-Galerkin (MLPG) approach in computational mechanics, Comput. Mech., 1998, 22, 117-127.

[99] Atluri S.N., Zhu T., New concepts in meshless methods, Int. J. Numer. Meth. Engng., 2000, 47, 537-556.

[100] Atluri S.N., Zhu T.L., The meshless local Petrov-Galerkin (MLPG) approach for solving problems in elasto-statics, Comput. Mech., 2000, 25, 169-179.

[101] Kansa E.J., Multiquadrics - A scattered data approximation scheme with applications to computational fluid-dynamics - I surface approximations and partial derivative estimates, Comput. Math. Appl., 1990, 19, 127-145.

[102] Kansa E.J., Multiquadrics - A scattered data approximation scheme with applications to computational fluid-dynamics - II solutions to parabolic, hyperbolic and elliptic partial differential equations, Comput. Math. Appl., 1990, 19, 147-161.

[103] Fasshauer G.E., Solving differential equations with radial basis functions: multilevel methods and smoothing”, Adv. Comput. Math., 1999, 11, 139-159.

[104] Ferreira A.J.M., Fasshauer G.E., Computation of natural frequencies of shear deformable beams and plates by an RBFpseudospectral method, Comput. Meth. Appl. Mech. Engng., 2006, 196, 134-146.

[105] Ferreira A.J.M., Fasshauer G.E., Batra R.C., Rodrigues J.D., Static deformations and vibration analysis of composite and sandwich plates using a layerwise theory and RBF-PS discretizations with optimal shape parameter, Compos. Struct., 
$2008,86,328-343$.

[106] Wendland H., Meshless Galerkin methods using radial basis functions, Math. Comput., 1999, 68, 1521- 1531.

[107] Buhmann M.D., Radial basis functions, Acta Numer., 2000, 9 , 1-38.

[108] Shu C., Ding H., Yeo K.S., Solution of partial differential equations by a global radial basis function- based differential quadrature method, Eng. Anal. Bound. Elem., 2004, 28, 1217 1226.

[109] Tornabene F., Fantuzzi N., Viola E., Ferreira A.J.M., Radial Basis Function method applied to doubly-curved laminated composite shells and panels with a general higher-order equivalent single Layer theory, Compos. Part B-Eng., 2013, 55, 642-659.

[110] Civan F., Sliepcevich C.M., Application of differential quadrature in solution of pool boiling in cavities, Proc. Oklahoma Acad. Sci., 1985, 65, 73-78.

[111] Chen W.L., A New Approach for Structural Analysis. The Quadrature Element Method, PhD Thesis, University of Oklahoma, 1994.

[112] Chen W.L., Striz A.G., Bert C.W., High accuracy plane stress and plate element in the quadrature element method, Int. J. Solids Struct., 2000, 37, 627-647.

[113] Zhong H., He Y., Solution of Poisson and Laplace equations by quadrilateral quadrature element. Int. J. Solids Struct., 1998, 35, 2805-2819.

[114] Shu C., Chew Y.T., Khoo B.C., Yeo K.S., Application of GDQ scheme to simulate incompressible viscous flows around complex geometries, Mech. Res. Commun., 1995, 22, 319-325.

[115] Shu C., Chew Y.T., Liu Y., An efficient approach for numerical simulation of flows in Czochralski crystal growth, J. Cryst. Growth, 1997, 181, 427- 436.

[116] Lam S.S.E., Application of the differential quadrature method to two-dimensional problems with arbitrary geometry. Comput. Struct., 1993, 47, 459-464.

[117] Bert C.W., Malik M., The differential quadrature method for irregular domains and application to plate vibration, Int. J. Mech. Sci., 1996, 38, 589-606.

[118] Chen C.-N., A generalized differential quadrature element method. Comput. Meth. Appl. Mech. Engrg, 2000, 188, 553 566.

[119] Chen C.-N., DQEM and DQFDM for the analysis of composite two-dimensional elasticity problems, Compos. Struct., 2013, 59, 3-13.

[120] Zhang Y.Y., Development of Differential Quadrature Methods and Their Applications to Plane Elasticity, PhD Thesis, National University of Singapore, 2003.

[121] Zong Z., Lam K., Zhang Y., A multidomain differential quadrature approach to plane elastic problems with material discontinuity, Math. Comput. Model., 2005, 41, 539-553.

[122] Zong Z., Zhang Y.Y., Advanced Differential Quadrature Methods, CRC Press, 2009.

[123] Xing Y., Liu B., High-accuracy differential quadrature finite element method and its application to free vibrations of thin plate with curvilinear domain, Int. J. Numer. Meth. Eng., 2009, 80, 1718-1742.

[124] Xing Y., Liu B., Liu G., A differential quadrature finite element method, Int. J. Appl. Mech., 2010, 2, 207-227.

[125] Zhong H., Yu T., A weak form quadrature element method for plane elasticity problems, Appl. Math. Model., 2009, 33, 38013814.
[126] Fantuzzi N., Generalized differential quadrature finite element method applied to advanced structural mechanics, PhD Thesis, University of Bologna, 2013.

[127] Viola E., Tornabene F., Fantuzzi N., Generalized differential quadrature finite element method for cracked composite structures of arbitrary shape, Compos. Struct., 2013, 106, 815-834.

[128] Viola E., Tornabene F., Ferretti E., Fantuzzi N., Soft core plane state structures under static loads using GDQFEM and Cell method, CMES, 2013, 94, 301-329.

[129] Viola E., Tornabene F., Ferretti E., Fantuzzi N., GDQFEM numerical simulations of continuous media with cracks and discontinuities, CMES, 2013, 94, 331-368.

[130] Viola E., Tornabene F., Ferretti E., Fantuzzi N., On static analysis of plane state structures via GDQFEM and Cell method, CMES, 2013, 94, 421-458.

[131] Fantuzzi N., Tornabene F., Strong formulation finite element method for arbitrarily shaped laminated plates - I. Theoretical analysis, Adv. Aircraft Space. Sci., 2014, 1, 124-142.

[132] Fantuzzi N., Tornabene F., Strong formulation finite element method for arbitrarily shaped laminated plates - II. Numerical analysis, Adv. Aircraft Space. Sci., 2014, 1, 143-173.

[133] Fantuzzi N., Tornabene F., Viola E., Generalized differential quadrature finite element method for vibration analysis of arbitrarily shaped membranes, Int. J. Mech. Sci., 2014, 79, 216251.

[134] Fantuzzi N., Tornabene F., Viola E., Ferreira A.J.M., A Strong Formulation Finite Element Method (SFEM) based on RBF and GDQ techniques for the static and dynamic analyses of laminated plates of arbitrary shape, Meccanica, 2014, 49, 2503-2542.

[135] Fantuzzi N., Tornabene F., Viola E., Four-parameter functionally graded cracked plates of arbitrary shape: a GDQFEM solution for free vibrations, Mech. Adv. Mat. Struct., 2014, (in press), DOI:

[136] Tornabene F., Fantuzzi N., Bacciocchi M., The strong formulation finite element method: stability and accuracy, Fract. Struct. Integr., 2014, 29, 251-265.

[137] Tornabene F., Fantuzzi N., Ubertini F., Viola E., Strong formulation finite element method based on differential quadrature: a survey, Appl. Mech. Rev., 2014, (in press), DOI:10.1115/1.4028859

[138] Zhong H., He Y., A note on incorporation of domain decomposition into the differential quadrature method, Commun. Numer. Methods Engrg., 2003, 19, 297-306.

[139] Bardell N.S., Langley R.S., Dunsdon J.M., On the free in-plane vibration of isotropic rectangular plates, J. Sound Vib., 1996, 191, 459-467.

[140] Hyde K., Chang J.Y., Bacca C., Wickert J.A., Parameter studies for plane stress in-plane vibration of rectangular plates, J. Sound Vib., 2001, 247, 471-487.

[141] Singh A.V., Muhammad T., Free in-plane vibration of isotropic non-rectangular plates, J. Sound Vib., 2004, 273, 219-231.

[142] Park C.I., Frequency equation for the in-plane vibration of a clamped circular plate, J. Sound Vib., 2008, 313, 325-333.

[143] Bashmal S., Bhat R., Rakheja S., In-plane free vibration of circular annular disks, J. Sound Vib., 2009, 322, 216-226.

[144] R.H. Macneal, R.L. Harder, A proposed standard set of problems to test finite element accuracy. Finite Elem. Anal Design, 1985, 1, 3-20.

[145] Rezaiee-Pajand, M. Karkon, An effective membrane element based on analytical solution, Eur. J. Mech. A/Solids, 2013, 39, 
268-279.

[146] M.C. Amirani, S. Khalili, N. Nemati, Free vibration analysis of sandwich beam with FG core using the element free Galerkin method, Compos. Struct.,2009, 90, 373-379.

[147] R. Cook, Concepts and applications of finite element analysis. Wiley, 2001.

[148] R. Cook, J. Avrashi, Error estimation and adaptive meshing for vibration problems, Comput. Struct., 1992, 44, 619-626.

[149] K.K. Gupta, Development of a finite dynamic element for free vibration analysis of two-dimensional structures. Int. J. Numer. Methods Engrg., 1978, 12, 1311-1327.

[150] Y. Li, N. Fantuzzi, F. Tornabene, On mixed mode crack initiation and direction in shafts: strain energy density factor and maximum tangential stress criteria, Eng. Fract. Mech., 2013, 109, 273-289.
[151] Y. Li, E. Viola, Size effect investigation of a central interface crack between two bonded dissimilar materials. Compos. Struct., 2013, 105, 90-107.

[152] E. Viola, N. Fantuzzi, A. Marzani, A. (2012): Cracks interaction effect on the dynamic stability of beams under conservative and nonconservative forces. Key Eng. Mat., 2012, 488-489, 383-386.

[153] E. Viola, Y. Li, N. Fantuzzi, N. (2012): On the stress intensity factors of cracked beams for structural analysis. Key Eng. Mat., 2012, 488-489, 379-382. 